\title{
Sum-Over-Histories Quantization of Relativistic Particle
}

\author{
Pavel Krtouš \\ Institute of Theoretical Physics, \\ Faculty of Mathematics and Physics, Charles University, \\ V Holešovičkách 2, 18000 Praha 8, Czech Republic
}

gr-qc/0011077

\begin{abstract}
Sum-over-histories quantization of particle-like theory in curved space is discussed. It is reviewed that the propagator satisfies the Schrödinger equation respective wave equation with a Laplace-like operator. The exact dependence of the operator on the choice of measure is shown.

Next, modifications needed for a manifold with a boundary are introduced, and the exact form of the equation for the propagator is derived. It is shown that the Laplace-like operator contains some distributional terms localized on the boundary. These terms induce proper boundary conditions for the propagator. This choice of boundary conditions is explained as a consequence of a measurement of particles on the boundary.

The interaction with sources inside of the domain and sources on the boundary is also discussed.
\end{abstract}




\section{Introduction}

The main goal of this work is to investigate a quantization of a relativistic particle using the sum-over-histories approach. We have the following motivations to do such a study.

The usual approach how to quantize a relativistic particle is the scalar field theory. Yet, it is a quantization of a completely different system - a quantization of a continuous field on spacetime. It is true that we can identify some states of such system as particle states - states with some properties of particles. But is there any other way to make a connection to the particle theory? Is it possible to quantize a classical relativistic particle, and does it give predictions equivalent to predictions of quantum scalar field theory?

There exists a candidate for the direct quantization of a particle theory - quantization using the sum-over-histories approach. The classical explanation of this approach for usual nonrelativistic physics can be found in [1] and more technically in [2, 4. A nice non-technical overview for a relativistic theory can be found in [5]. Beside these classical introductions, this approach has received considerable attention in recent years (see for example [6]). The new development has led to a generalization of this method called generalized quantum mechanics (see [6] 8]).

In this approach the transition amplitudes associated with the chosen criteria are computed by summing over amplitudes of all possible histories which meet the criteria. It is known that some of these amplitudes computed for a relativistic particle lead in the special cases (e.g., in flat spacetime) to quantities which can also be obtained from scalar field theory. The goal of our work is to investigate this correspondence in more detail.

There are more reasons for studying relativistic particle theory. One of the attempts to understand the quantization of the gravitational field coupled to matter is to reduce the full gravitation theory to a system with a finite number of degrees of freedom and try to quantize this simplified system. These reduced theories are called minisuperspace models. It is well known that such reduced system is essentially equivalent to a particle theory in a Lorentzian space with (usually) a complicated potential. A common method for the quantization of minisuperspace models is the sum-over-histories approach.

Key feature of our investigation is that we study the particle theory on a bounded domain of spacetime, and that we pay an attention to the exact form of boundary conditions. The usual approach is a bit generous on this question - the theory is usually formulated on the whole spacetime with not always clearly formulated special behavior at infinities. In the flat spacetime such an approach is justifiable because there exists a preferred behavior at infinities, but in a general curved spacetime we have to be more careful. The question of boundary conditions is usually completely ignored in definitions of the path integral. We try to formulate the theory in a more careful way and identify its boundary-condition dependence.

The plan of our work is the following. In the first part of the paper we review sum-over-histories approach to the quantization of the particle in curved spacetime without boundary. Equations for key amplitudes (the propagator and Feynmann Green function) are derived and their exact dependence on the definition of the path integral is shown.

It the second part we investigate the theory on a bounded domain. On the bounded domain we have to modify our definition of the path integral. The new definition leads to a modification of the equations for physical amplitudes. Some new distributional terms localized on the boundary 
appear in these equation and we find that they specify the exact form of boundary conditions for the amplitudes. It is shown that the exact form of the boundary conditions depends on the details of the definition of the path integral.

Finally we discuss shortly a physical meaning of the boundary conditions. We argue that boundary conditions can serve as a phenomenological description of apparatuses measuring particles on the boundary of the domain.

We will continue in this discussion in the following paper [9], where a relationship of different boundary conditions will be investigated and a connection with a particle definition in the quantum scalar field theory will be shown.

Sections 1 and 2 contain the main line of arguments, details of computations can be found in the appendix A. The appendix B contains a general overview of the geodesic theory necessary for the computations, including not common theory for reflected geodesics.

Let us note that our study can accommodate a wider range of theories. The theory will be parameterized by two signature factors. One of them (the factor $n$ ) characterizes a signature of a target space metric (the metric in which a particle lives), and another (the factor $\nu$ ) describes whether the theory is physical or Euclidian. For real $n$ and imaginary $\nu$ we obtain non-relativistic particle in curved space, for $n$ and $\nu$ both imaginary we obtain relativistic particle in curved spacetime and for real signature factors we get mathematically better behaving, but non-physical Euclidian version of the theory. Usually most quantities are well defined in the Euclidian sector and the definition for physical signature are obtained by an analytical continuation in the signature factors. 


\section{Particle in a curved space without boundary}

\section{Space of histories, action and amplitudes}

In this section we shortly formulate the sum-over-histories approach to a quantization of a particlelike theory. A similar calculation have been done for example in [10, 11]. We present our derivation here because we will generalize our line of reasoning in then next section to the case when a particle is moving in a domain with a boundary.

As usual in the sum-over-histories approach, the theory is characterized by a space of histories and an action. An elementary history in our case is a trajectory - an imbedding of a 1-dimensional manifold $N$ (called the inner space) to a $d$-dimensional spacetime manifold $M$ - and an inner space metric $h$ on the inner manifold $N$. $M$ is equipped with a spacetime metric $g$ and scalar potential $V$.

In the Euclidian version of the theory $h$ is positive definite; in the physical version it is gegative definite. In the Euclidian version we allow the spacetime metric to be also positive definitel.

The whole theory is invariant under diffeomorphisms of the inner manifold $N$. As usual (e.g. [12]), we factorize over this symmetry. If we fix a coordinate $\eta: N \rightarrow\langle 0,1\rangle$ on the inner manifold $N$ we can characterize a class of equivalent histories using a pair $[\boldsymbol{x}, \tau]$, where $\boldsymbol{x}$ is a map $\boldsymbol{x}:\langle 0,1\rangle \rightarrow M$ and $\tau$ is a total inner time or a total inner length of $N$ measured using the inner metric $h$.

The Euclidian actiont in these variables has the form

$$
I(\tau, \boldsymbol{x})=\frac{1}{2} \int_{\langle 0,1\rangle}\left(\frac{1}{\nu \tau} \dot{\boldsymbol{x}}^{\boldsymbol{\alpha}} \dot{\boldsymbol{x}}^{\boldsymbol{\beta}} g_{\boldsymbol{\alpha} \boldsymbol{\beta}}(\boldsymbol{x})+\nu \tau V(\boldsymbol{x})\right) d \eta \quad .
$$

Here $\nu$ is a constant signature factor distinguishing Euclidian and physical versions of the theory. In the former case $\nu=2$, in the later it is multiplied by $i$.

In the sum-over-histories approach to quantum theory we can define an amplitude $A(\mathrm{H})$ for any set of histories H by "summing" over amplitudes of elementary histories in the set. The quantum amplitude is not directly a physical measurable quantity. We need an additional notion of distinguishable or decoherent histories to give a probabilistic interpretation to the square of amplitudes. We expect that this notion has the same symmetry as the action and a measure on histories. This means that we will be always interested in amplitudes of sets of histories which are invariant under the action of the diffeomorphism group. For such sets we can factorize the path integral and eliminate the reference to the diffeomorphism (e.g. 12]). In the factorized integral we are summing only over variables $[\boldsymbol{x}, \tau]$ :

$$
A(\mathrm{H})=\int_{[\tau, \boldsymbol{x}] \in \mathrm{H}} \mathfrak{M}_{\text {red }}(\tau, \boldsymbol{x}) \exp (-I(\tau, \boldsymbol{x})),
$$

with a reduced, renormalized measure $\mathfrak{M}_{\text {red }}$.

\section{Propagator}

It is useful to compute an amplitude $\frac{1}{n} K\left(\tau, x_{\mathrm{f}} \mid x_{\mathrm{i}}\right)$ - called the propagator or heat kernel - for the set of histories restricted only by positions of end points of the trajectory $x_{\mathrm{f}}$ and $x_{\mathrm{i}}$ in the spacetime 
$M$ and by fixing an inner time to a particular value $\tau$ :

$$
\frac{1}{n} K\left(\tau, x_{\mathrm{f}} \mid x_{\mathrm{i}}\right)=\int_{\boldsymbol{x} \in \mathcal{T}\left(x_{\mathrm{f}} \mid x_{\mathrm{i}}\right)} \mathfrak{M}^{F}\left(\tau, x_{\mathrm{f}} \mid x_{\mathrm{i}}\right)[\boldsymbol{x}] \exp (-I(\tau, \boldsymbol{x})),
$$

where $\mathcal{T}\left(x_{\mathrm{f}} \mid x_{\mathrm{i}}\right)$ is a set of trajectories $\boldsymbol{x}:\langle 0,1\rangle \rightarrow M$ with $\boldsymbol{x}(1)=x_{\mathrm{f}}$ and $\boldsymbol{x}(0)=x_{\mathrm{i}}$ and $n$ is a constant factor 3 governing signature of the spacetime metric $g$.

Because the set of histories $\left[\tau, \mathcal{T}\left(x_{\mathrm{f}} \mid x_{\mathrm{i}}\right)\right]=\{\tau\} \times \mathcal{T}\left(x_{\mathrm{f}} \mid x_{\mathrm{i}}\right)$ is a lower dimensional subset of the space of all histories, $K\left(\tau, x_{\mathrm{f}} \mid x_{\mathrm{i}}\right)$ is essentially an amplitude "density" on the space $\mathbb{R}^{+} \times M \times M$ of values $\left[\tau, x_{\mathrm{f}}, x_{\mathrm{i}}\right]$. Therefore we have to expect that the restriction $\mathfrak{M}^{F}\left(\tau, x_{\mathrm{f}} \mid x_{\mathrm{i}}\right)$ of the measure $\mathfrak{M}_{\text {red }}$ to the space $\left[\tau, \mathcal{T}\left(x_{\mathrm{f}} \mid x_{\mathrm{i}}\right)\right]$, which we call the Feynman measure, depends on $\tau$ and end points $x_{\mathrm{f}}$ and $x_{\mathrm{i}}$; maybe only in a "trivial" way.

In other words, an amplitude density of an elementary history on the space $\left[\tau, \mathcal{T}\left(x_{\mathrm{f}} \mid x_{\mathrm{i}}\right)\right]$ is

$$
A(\tau, \boldsymbol{x})=\mathfrak{M}^{F}\left(\tau, x_{\mathrm{f}} \mid x_{\mathrm{i}}\right)[\boldsymbol{x}] \exp (-I(\tau, \boldsymbol{x})) .
$$

It is well known 28, 6, 10, 11] that with the right choice of the measure $\mathfrak{M}^{F}\left(\tau, x_{\mathrm{f}} \mid x_{\mathrm{i}}\right)$ the propagator satisfies the equationt

$$
-\dot{K}(\tau)=\frac{\nu}{2} \mathrm{~F} \bullet K(\tau) \quad, \quad K(0)=\mathcal{G}^{-1},
$$

where $\mathrm{F}$ is $\mathrm{g}$ wave operator fixed by the action and the measure (see later), $\mathcal{G}=\mathfrak{g}^{\frac{1}{2}} \delta$ is a delta distribution $\mathrm{E}$ on $M$ normalized to the metric volume element $\mathfrak{g}^{\frac{1}{2}}$. In other words, $K$ is the exponential of $\mathrm{F}$

$$
K(\tau)=\exp \left(-\frac{\nu \tau}{2} \mathrm{~F}\right) \cdot \mathcal{G}^{-1}
$$

We have not specified the "right choice" of the measure yet. It can be a very problematic task from the pure mathematical point of view. Instead of trying to develop a measure theory on infinite dimensional spaces for oscillatory integrals (where the main problem lies), we take the usual approach of formal manipulations, and we define the measure by its decomposition properties and approximation for small time intervals. The former is given in equation (1.11), and the latter is given in equation (1.20).

The idea of the proof of the relations $(1.5)$ is in proving key properties of the exponential,

$$
\begin{gathered}
K\left(\tau_{\mathrm{f}}\right) \bullet \mathcal{G} \bullet K\left(\tau_{\mathrm{i}}\right)=K\left(\tau_{\mathrm{f}}+\tau_{\mathrm{i}}\right), \\
\mathcal{G} \bullet K(\tau) \bullet \mathcal{G}=\mathcal{G}-\frac{\nu \tau}{2} \mathcal{F}+\mathcal{O}\left(\tau^{2}\right),
\end{gathered}
$$

where $\mathcal{F}=\mathcal{G} \bullet \mathrm{F}$ is the quadratic form of the differential operator $\mathrm{F}$.

\section{Composition law}

The first condition (1.7) is a composition law for the amplitude $K$. This law reflects the possibility to decompose a history $[\tau, \boldsymbol{x}]$ into histories $\left[\tau_{\mathrm{i}}, \boldsymbol{x}_{\mathrm{i}}\right]$ during an initial amount of inner time $\tau_{\mathrm{i}}$ and $\left[\tau_{\mathrm{f}}, \boldsymbol{x}_{\mathrm{f}}\right]$ during a final amount of inner time $\tau_{\mathrm{f}}$. We say that a history $[\tau, \boldsymbol{x}]=\left[\tau_{\mathrm{f}}, \boldsymbol{x}_{\mathrm{f}}\right] \odot\left[\tau_{\mathrm{i}}, \boldsymbol{x}_{\mathrm{i}}\right]$ is given by joining of histories $\left[\tau_{\mathrm{f}}, \boldsymbol{x}_{\mathrm{f}}\right]$ and $\left[\tau_{\mathrm{i}}, \boldsymbol{x}_{\mathrm{i}}\right]$ if

$$
\tau=\tau_{\mathrm{f}}+\tau_{\mathrm{i}} \quad, \quad \boldsymbol{x}_{\mathrm{f}}(0)=\boldsymbol{x}_{\mathrm{i}}(1) \quad, \quad \boldsymbol{x}_{\mathrm{f}}=\boldsymbol{x}\left(\frac{\tau_{\mathrm{i}}+\eta \tau_{\mathrm{f}}}{\tau}\right) \quad, \quad \boldsymbol{x}_{\mathrm{i}}=\boldsymbol{x}\left(\frac{\eta \tau_{\mathrm{i}}}{\tau}\right)
$$

The actions is additive with respect of joining histories. 
We have a natural decomposition of the set of histories $\left[\tau, \mathcal{T}\left(x_{\mathrm{f}} \mid x_{\mathrm{i}}\right)\right]$ which defines the propagator $K\left(\tau, x_{\mathrm{f}} \mid x_{\mathrm{i}}\right)$ to disjoint sets $\left[\tau_{\mathrm{f}}, \boldsymbol{\mathcal { T }}\left(x_{\mathrm{f}} \mid x_{\mathrm{o}}\right)\right] \times\left[\tau_{\mathrm{i}}, \mathcal{T}\left(x_{\mathrm{o}} \mid x_{\mathrm{i}}\right)\right]$

$$
\left[\tau, \mathcal{T}\left(x_{\mathrm{f}} \mid x_{\mathrm{i}}\right)\right]=\bigcup_{x_{\mathrm{o}} \in M}\left[\tau_{\mathrm{f}}, \mathcal{T}\left(x_{\mathrm{f}} \mid x_{\mathrm{o}}\right)\right] \times\left[\tau_{\mathrm{i}}, \mathcal{T}\left(x_{\mathrm{o}} \mid x_{\mathrm{i}}\right)\right]
$$

If the measures on these sets are related by

$$
\mathfrak{M}^{F}\left(\tau, x_{\mathrm{f}} \mid x_{\mathrm{i}}\right)[\boldsymbol{x}]=\mathfrak{M}^{F}\left(\tau_{\mathrm{f}}, x_{\mathrm{f}} \mid x_{\mathrm{o}}\right)\left[\boldsymbol{x}_{\mathrm{f}}\right] n \mathfrak{g}^{\frac{1}{2}}\left(x_{\mathrm{o}}\right) \mathfrak{M}^{F}\left(\tau_{\mathrm{i}}, x_{\mathrm{o}} \mid x_{\mathrm{i}}\right)\left[\boldsymbol{x}_{\mathrm{i}}\right],
$$

we get (1.7) by a straigthforward calculation.

The condition (1.11) represents a reasonable assumption of the locality of the measure $\mathfrak{M}^{F}$. Together with the additivity of the action it reflects the rule of the sum-over-histories approach to quantum mechanics - that the amplitude of independent (here consequent) events is given by multiplication of individual amplitudes. This condition is the first part of our definition of the measure. Now we know how to construct the measure $\mathfrak{M}^{F}(\tau)$ for some time $\tau$ from measures for shorter time intervals. To conclude the definition of the measure, we need to specify it for an infinitesimally short inner time interval. This moves us to an investigation of the short time behavior of the heat kernel.

\section{Short time amplitude}

Now we turn to prove equation (1.8). It can be found in the literature (e.g. 10,11]), but we present it here to show how the measure is actually determined and how the operator $\mathcal{F}$ depends on this choice.

We ignore technical difficulties in the definition of the path integral, and we assume that this integral has most of the properties of a usual integral in a finite-dimensional manifold. This allows us to find the short time behavior for the propagator.

First we write an expansion of the action for small $\tau$

$$
I(\tau, \boldsymbol{x})=\frac{1}{\tau} I_{-1}(\boldsymbol{x})+I_{0}(\boldsymbol{x})+\tau I_{1}(\boldsymbol{x})+\ldots .
$$

For the action we are using it means

$$
\begin{aligned}
I_{-1}(\boldsymbol{x}) & =\frac{1}{2 \nu} \int_{\eta \in\langle 0,1\rangle} \dot{\boldsymbol{x}}^{\boldsymbol{\alpha}} \dot{\boldsymbol{x}}^{\boldsymbol{\beta}} g_{\boldsymbol{\alpha} \boldsymbol{\beta}}(\boldsymbol{x}) d \eta, \\
I_{0}(\boldsymbol{x}) & =0, \\
I_{1}(\boldsymbol{x}) & =\frac{\nu}{2} \int_{\eta \in\langle 0,1\rangle} V(\boldsymbol{x}) d \eta .
\end{aligned}
$$

We assume that the measure is slowly changing in $\tau$ compared to the leading term in the action.

The dominant contribution to the integral (1.3) comes from an extremum $\overline{\boldsymbol{x}}\left(x_{\mathrm{f}} \mid x_{\mathrm{i}}\right)$ of the leading term $I_{-1}$ in the exponent. But the extremum of the functional (1.13) is clearly a geodesic of the metric $g$. We expand all expressions around this extremum

$$
\boldsymbol{x}=\overline{\boldsymbol{x}}\left(x_{\mathrm{f}} \mid x_{\mathrm{i}}\right)+\sqrt{\tau} \overrightarrow{\boldsymbol{x}},
$$

where $\overrightarrow{\boldsymbol{x}}$ is a tangent vector to the space of trajectories $\mathcal{T}\left(x_{\mathrm{f}} \mid x_{\mathrm{i}}\right)$ at the extremum $\overline{\boldsymbol{x}}\left(x_{\mathrm{f}} \mid x_{\mathrm{i}}\right)$. We actually need to specify what the addition in the last equation means. It will be done more carefully 
in a similar situation in appendix A (see eq. (A.5)). But now we are interested more in a qualitative answer, so we skip these details here. The expanded integral (1.3) has the structure

$$
\begin{aligned}
& \frac{1}{n} K\left(\tau, x_{\mathrm{f}} \mid x_{\mathrm{i}}\right)=\exp \left(-I\left(\overline{\boldsymbol{x}}\left(x_{\mathrm{f}} \mid x_{\mathrm{i}}\right)\right)\right) \int_{\overrightarrow{\boldsymbol{x}} \in \mathbf{T}_{\overline{\boldsymbol{x}}} \mathcal{T}} \mathfrak{M}^{F}{ }_{*}\left(\tau, x_{\mathrm{f}} \mid x_{\mathrm{i}}\right) \exp \left(-\frac{1}{2} \overrightarrow{\boldsymbol{x}} \cdot \delta^{2} I_{-1}\left(\overline{\boldsymbol{x}}\left(x_{\mathrm{f}} \mid x_{\mathrm{i}}\right)\right) \cdot \overrightarrow{\boldsymbol{x}}\right) \times \\
& \times\left(1+\sqrt{\tau}\left(\overrightarrow{\boldsymbol{x}}^{\text {odd }}-\text { terms }\right)+\tau\left(\overrightarrow{\boldsymbol{x}}^{\text {even }}-\text { terms }\right)+\ldots\right) .
\end{aligned}
$$

Here $\mathfrak{M}^{F}{ }_{*}\left(\tau, x_{\mathrm{f}} \mid x_{\mathrm{i}}\right)$ is a leading term in the $\tau$ and $\overrightarrow{\boldsymbol{x}}$-expansion of the measure $\mathfrak{M}^{F}\left(\tau, x_{\mathrm{f}} \mid x_{\mathrm{i}}\right)$ after change of variables $\boldsymbol{x} \rightarrow \overrightarrow{\boldsymbol{x}}$. $\mathfrak{M}^{F}{ }_{*}$ is a constant measure on the tangent vector space to the space of trajectories $\boldsymbol{T}\left(x_{\mathrm{f}} \mid x_{\mathrm{i}}\right)$. The actual dependence on $\overrightarrow{\boldsymbol{x}}$ is hidden in higher terms of the $\overrightarrow{\boldsymbol{x}}$-expansion. As a leading term in the $\tau$-expansion, $\mathfrak{M}^{F}{ }_{*}$ depends on $\tau$ in a trivial way - it is proportional to a power of $\tau$. Of course, this statement is formal - the exponent of $\tau$ in $\mathfrak{M}^{F}{ }_{*}$ is of the order of the dimension of the tangent space, which is infinite.

" $\overrightarrow{\boldsymbol{x}}$-terms" in the last equation represents terms resulting from the expansion of the action and the measure; $\overrightarrow{\boldsymbol{x}}^{\text {odd }}$ or $\overrightarrow{\boldsymbol{x}}^{\text {even }}$ suggest that $\overrightarrow{\boldsymbol{x}}$ occurs in these terms in odd or even power. For convenience we combined the term $\tau I_{1}$ into the prefactor despite the fact that it could be included among terms proportional to $\tau$.

The value $\nu I_{-1}\left(\overline{\boldsymbol{x}}\left(x_{\mathrm{f}} \mid x_{\mathrm{i}}\right)\right)$ is a well-known quantity called the world function, or half the squared geodesic distancet

$$
\sigma\left(x_{\mathrm{f}} \mid x_{\mathrm{i}}\right)=\nu I_{-1}\left(\overline{\boldsymbol{x}}\left(x_{\mathrm{f}} \mid x_{\mathrm{i}}\right)\right)=\frac{1}{2} \int_{\langle 0,1\rangle} \dot{\dot{\boldsymbol{x}}}\left(x_{\mathrm{f}} \mid x_{\mathrm{i}}\right) \cdot g\left(\overline{\boldsymbol{x}}\left(x_{\mathrm{f}} \mid x_{\mathrm{i}}\right)\right) \cdot \dot{\overline{\boldsymbol{x}}}\left(x_{\mathrm{f}} \mid x_{\mathrm{i}}\right) d \eta \quad .
$$

We also use the notation

$$
\bar{V}\left(x_{\mathrm{f}} \mid x_{\mathrm{i}}\right)=\frac{2}{\nu} I_{1}\left(\overline{\boldsymbol{x}}\left(x_{\mathrm{f}} \mid x_{\mathrm{i}}\right)\right)=\int_{\langle 0,1\rangle} V\left(\overline{\boldsymbol{x}}\left(x_{\mathrm{f}} \mid x_{\mathrm{i}}\right)\right) d \eta \quad .
$$

The integral (1.17) is a simple Gaussian integration. (In fact, one approach to defining infinitedimensional integrals is through the definition of a "Gaussian" measure which in our case would be $\left.\mathfrak{M}^{F}{ }_{*} \exp \left(-\frac{1}{2} \overrightarrow{\boldsymbol{x}} \cdot \delta^{2} I_{-1} \cdot \overrightarrow{\boldsymbol{x}}\right)\right)$. The integration can be performed, at least formally. If the measure $\mathfrak{M}^{F}{ }_{*}(\tau)$ has the already mentioned $\tau$-dependence, the result can be written

$$
\begin{aligned}
K\left(\tau, x_{\mathrm{f}} \mid x_{\mathrm{i}}\right)=\frac{n}{(2 \pi \nu \tau)^{\frac{d}{2}}} \Delta\left(x_{\mathrm{f}} \mid x_{\mathrm{i}}\right)( & \left.\alpha_{0}\left(x_{\mathrm{f}} \mid x_{\mathrm{i}}\right)-\tau \frac{\nu}{2} \alpha_{1}\left(x_{\mathrm{f}} \mid x_{\mathrm{i}}\right)+\mathcal{O}\left(\tau^{2}\right)\right) \times \\
& \times \exp \left(-\frac{1}{\nu \tau} \sigma\left(x_{\mathrm{f}} \mid x_{\mathrm{i}}\right)-\frac{\nu \tau}{2} \bar{V}\left(x_{\mathrm{f}} \mid x_{\mathrm{i}}\right)\right),
\end{aligned}
$$

where $\alpha_{0}\left(x_{\mathrm{f}} \mid x_{\mathrm{i}}\right)$ should satisfy

$$
\alpha_{0}(x \mid x)=1
$$

Here $\Delta\left(x_{\mathrm{f}} \mid x_{\mathrm{i}}\right)$ is Van Vleck-Morette determinant (see (B.13)).

The terms proportional to $\sqrt{\tau}$ disappeared during integration, thanks to the odd power of $\overrightarrow{\boldsymbol{x}}$. The particular behaviour a coincidence limit of the coefficient $\alpha_{0}$ will be needed for a proper normalization in (1.5). To obtain this behaviour we need the mentioned $\tau$-dependence of the measure, which can be expressed by condition

$$
\mathfrak{M}^{F}{ }_{*}(\tau, x \mid x) \operatorname{Det}\left(\frac{\delta^{2} I_{-1}(\overline{\boldsymbol{x}}(x \mid x))}{2 \pi \nu \tau}\right)^{-\frac{1}{2}}=\frac{1}{(2 \pi \nu \tau)^{\frac{d}{2}}}
$$


I.e., the meassure must satisfy this condition to conlude proof of equation (1.8).

Coefficients in front of powers of $\tau$ could be expressed in terms of variations of the action and the measure. But because we did not specify the measure precisely yet, we can do it now by fixing these coefficients. I.e., we can define the measure $\mathfrak{M}^{F}$ by choosing functions $\alpha_{0}\left(x_{\mathrm{f}} \mid x_{\mathrm{i}}\right)$ and $\alpha_{1}\left(x_{\mathrm{f}} \mid x_{\mathrm{i}}\right)$.

In the following we will prove that a form of the operator $\mathcal{F}$ in (1.8) depends only on the coincidence limits of $\alpha_{1}$ and the first two derivatives of $\alpha_{0}$. So we can ignore terms with higher power of $\tau$ in eq. (1.20). As discussed before, equations (1.8) together with composition law (1.7) determines the propagator $K$, i.e. also all important information hidden in the measure $\mathfrak{M}^{F}$. This means that a knowledge of the mentioned coincidence limits concludes our definition of the measure and path integral itself.

Let us note that this argument is some kind of justification of the usual time-discretization of the path integral and of a priori choice of the short time amplitude in the form (1.20). But in principle it would be possible to define the measure $\mathfrak{M}^{F}$ in some more compact way and compute exactly the form of the functions $\alpha_{0}$ and $\alpha_{1}$ in terms of variation of the action and the measure.

\section{Short time behavior of the heat kernel}

Now we continue with the proof of equation (1.8). We show that for small $\tau$ the amplitude (1.20) has the desired behavior in a distributional sense. Most of the technical work is done in appendix A where it is shown that for small $\tau$ the following expansion holds (equation (A.8))

$$
\begin{aligned}
\frac{n}{(2 \pi \nu \tau)^{\frac{d}{2}}} \int_{x, z \in M} \mathfrak{g}^{\frac{1}{2}}(x) \mathfrak{g}^{\frac{1}{2}}(z) \Delta(x \mid z) & \exp \left(-\frac{1}{\nu \tau} \sigma(x \mid z)\right) \varphi(x) \psi(z)= \\
& =\varphi \bullet \mathcal{G} \bullet \psi-\tau \frac{\nu}{2} \varphi \bullet \mathcal{L} \bullet \psi+\mathcal{O}\left(\tau^{2}\right),
\end{aligned}
$$

where $\varphi$ and $\psi$ are smooth test functions and $\mathcal{L}$ is the Laplace operator quadratic form

$$
\begin{aligned}
\varphi \cdot \mathcal{L} \bullet \psi & =\int_{M} \mathfrak{g}^{\frac{1}{2}}(\mathrm{~d} \varphi) \cdot g^{-1} \cdot(\mathrm{d} \psi)= \\
& =-\int_{M} \mathfrak{g}^{\frac{1}{2}} \varphi\left(\nabla^{2} \psi\right)=-\int_{M} \mathfrak{g}^{\frac{1}{2}} \psi\left(\nabla^{2} \varphi\right) .
\end{aligned}
$$

Let us remember that now we are discussing the case of a manifold without boundary, and therefore we do not have to worry about boundary conditions for the Laplace operator and integration by parts.

Using this result it is easy to show that

$$
\begin{aligned}
& \varphi \cdot \mathcal{G} \bullet K(\tau) \cdot \mathcal{G} \bullet \psi= \\
& =\frac{n}{(2 \pi \nu \tau)^{\frac{d}{2}}} \int_{x, z \in M} \mathfrak{g}^{\frac{1}{2}}(x) \mathfrak{g}^{\frac{1}{2}}(z) \Delta(x \mid z)\left(\alpha_{0}(x \mid z)-\tau \frac{\nu}{2} \alpha_{1}(x \mid z)+\mathcal{O}\left(\tau^{2}\right)\right) \times \\
& \times \exp \left(-\frac{1}{\nu \tau} \sigma(x \mid z)-\frac{\nu \tau}{2} \bar{V}(x \mid z)\right) \varphi(x) \psi(z)= \\
& =\varphi \bullet \mathcal{G} \bullet \psi- \\
& -\tau \frac{\nu}{2} \varphi \bullet\left(\mathcal{L}+(V \mathcal{G})+\left(g^{-1 \mu \nu}\left[\mathrm{d}_{1 \mu} \mathrm{d}_{\mathrm{r} \nu} \alpha_{0}\right] \mathcal{G}\right)+\left(\left[\alpha_{1}\right] \mathcal{G}\right)\right) \bullet \psi- \\
& -\tau \frac{\nu}{2} \varphi \cdot\left(\left(\tilde{\mathrm{d}}_{\boldsymbol{\mu}} g^{-1 \mu \nu}\left[\mathrm{d}_{1 \nu} \alpha_{0}\right]\right) \cdot \mathcal{G}+\mathcal{G} \bullet\left(\left[\mathrm{d}_{\boldsymbol{r} \boldsymbol{\mu}} \alpha_{0}\right] g^{-1 \mu \nu} \stackrel{\mathrm{d}}{\nu}_{\nu}\right)\right) \bullet \psi+ \\
& +\mathcal{O}\left(\tau^{2}\right) \text {, }
\end{aligned}
$$


where we used $[\bar{V}]=V$ and $\left[\alpha_{0}\right]=1$. Here the $[A]$ denotes a coincidence limit of a bitensor $A, \mathrm{~d}_{\mathrm{r}} A$ and $\mathrm{d}_{1} A$ are derivatives with respect of the right and left arguments and the bi-distributions $\tilde{\mathrm{d}}$ and $\widetilde{d}$ are derivatives acting to the left and to the right

If the condition

$$
\left[\mathrm{d}_{\mathrm{r}} \alpha_{0}\right]=\left[\mathrm{d}_{1} \alpha_{0}\right]=0
$$

is satisfied, we see that the propagator $K(\tau)$ has really the form (1.8) with

$$
\begin{gathered}
\mathcal{F}=\mathcal{L}+\mathcal{V}, \\
\mathcal{V}=\left(V+\left[\alpha_{1}\right]+g^{-1 \mu \nu}\left[\mathrm{d}_{1 \mu} \mathrm{d}_{r \nu} \alpha_{0}\right]\right) \mathcal{G} .
\end{gathered}
$$

I.e., $\mathcal{F}$ is a Laplace operator with a potential term which include the original potential $V$ from the action and additional parts depending on the choice of the measure.

A common choice for $\alpha_{0}$ is a power of the Van Vleck-Morette determinant

$$
\alpha_{0}=\Delta^{-p},
$$

which satisfies the condition (1.25). It leads to an additional part in the potential,

$$
g^{-1 \mu \nu}\left[\mathrm{d}_{1 \mu} \mathrm{d}_{r \nu} \Delta^{-p}\right]=\frac{p}{3} R,
$$

where $R$ is a scalar curvature of the metric $g$.

The condition (1.25) is actually a consequence of (1.21) and an assumption of the symmetry of $\alpha_{0}$

$$
\alpha_{0}(x \mid z)=\alpha_{0}(z \mid x)
$$

It is a natural assumption in the case when the theory is symmetric under trajectory reversal. However this condition does not have to be satisfied if there is a preferred path direction as for example in the case of interaction with an electromagnetic field. But we will not discuss such a situation, and in the following we will assume that the conditions $(1.30)$ and $(1.25)$ are satisfied.

In summary, we have seen that for small $\tau$ the propagator $K(\tau)$ has the behavior given by $(1.8)$. If the measure is defined using the decomposition property (1.7) and the short time amplitude (1.20), the operator $\mathcal{F}$ is fixed by knowledge of the coincidence limits of $\alpha_{1}$ and the first two derivatives of $\alpha_{0}$.

\section{Feynman Green Function}

For the relativistic particle the inner time is physically undetectable and therefore any physical set of histories will include elementary histories with all possible inner times. Therefore we are interested in the amplitude called the Feynman Green function $\frac{1}{n} G^{F}\left(x_{\mathrm{f}} \mid x_{\mathrm{i}}\right)$ associated with the set of histories restricted only by the initial and final points $x_{\mathrm{f}}, x_{\mathrm{i}}$. We can obtain it from the propagator $\frac{1}{n} K\left(\tau, x_{\mathrm{f}} \mid x_{\mathrm{i}}\right)$ by summing over all possible inner times $\tau$ using the measure $\frac{\nu}{2} d \tau$

$$
\frac{1}{n} G^{F}\left(x_{\mathrm{f}} \mid x_{\mathrm{i}}\right)=\int_{\mathbb{R}^{+}} \frac{1}{n} K\left(\tau, x_{\mathrm{f}} \mid x_{\mathrm{i}}\right) \frac{\nu}{2} d \tau
$$

Using eq. (1.6) we immediately get that the Feynman Green function is the inverse of the wave operator $\mathcal{F}$. 


\section{Boundary conditions}

In this section we completely ignored the question of boundary conditions using as excuse that we are working in a manifold without boundary. Certainly this is correct, if the manifold $M$ is compact. But it is also correct in the case of a non-compact manifold with a sufficiently "nice" metric $g$ at infinities. In such cases there exists a canonical choice of boundary conditions for differential operators used above, and these boundary conditions usually allow us to integrate by parts. But problem arise for the relativistic particle when the manifold $M$ is Lorentzian and operators as $\mathcal{L}$, $\mathcal{F}$ are hyperbolic. In this case the choice of boundary conditions at the temporal infinities plays an important physical role, and it is worth further investigation. First let us note that in a special situations (e.g. existence of a time-like Killing vector in the distant past and future) a canonical choice of boundary conditions still exists. But canonical here essentially means the most natural physical choice. In a general spacetime we do not have this special choice, and we have to address the question of the boundary conditions. To deal with this problem, in the next section we will investigate our theory in a bounded domain $\Omega$ of the manifold $M$. 


\section{Particle in a curved space with boundary}

\section{General consideration}

In this section our goal is a better understanding of the physical meaning of boundary conditions for the differential operators in the equation for the propagator and Green function. Therefore we restrict ourselves to a domain $\Omega$ in the manifold $M$ which is bounded "in all physically interesting directions". This means that we will investigate the boundary conditions on boundaries which are not at infinity.

If the target space metric is positive definite (i.e. in Euclidian and non-relativistic version of the theory) all problems with infinities could be solved by a restriction to a compact domain. But in the case of relativistic particle, which we are mostly intersted in, we also allow domains which do not have to be compact, we allow the domain $\Omega$ to be unbounded if we know that its infinity is "safe".

The situation we have in the mind is the Lorentzian globally hyperbolic manifold with asymptotically flat spatial infinity. In this case we can ignore spatial infinity because it makes a sense to restrict ourselves to situations in which spacetime is "empty" sufficiently far in space directions. But because of the hyperbolic nature of the evolution equation we cannot ignore boundary conditions in the time directions. They represent "initial" and "final" conditions of the system. And we want to understand exactly this relationship.

Therefore in the case of a Lorentzian globally hyperbolic manifold, the typical choice of the domain will be a sandwich domain between two Cauchy surfaces. But whole discussion is also valid for Euclidian version of the theory restricted to a compact domain.

\section{Restriction to a domain - naive approach}

Let us start with a straighforward restriction to a domain $\Omega$. We want to compute an amplitude $K_{\mathrm{o}}\left(\tau, x_{\mathrm{f}} \mid x_{\mathrm{i}}\right)$ which corresponds to a set of histories with inner time $\tau$, endpoints $x_{\mathrm{i}}, x_{\mathrm{f}}$ and which wholly belong to the domain $\Omega$. We can repeat the derivation of the short time amplitude $(1.20)$, at least for $x_{\mathrm{f}}, x_{\mathrm{i}}$ sufficiently far from the boundary, because for small $\tau$ only trajectories near to the geodesic between $x_{\mathrm{f}}$ and $x_{\mathrm{i}}$ contribute to the amplitude.

If we do this calculation, we find that there is a new term in the expansion. As can be seen in eq. (A.7), the smoothed short time amplitude leads to a Gauss integration in a variable $Z$ from a tangent space at a point $x$, and in the case of a space without boundary the integration of odd powers of $Z$ disappears. But in the case of a domain with a boundary for a point $x$ near the boundary the Gauss integration is not always over the whole tangent space and therefore the integral of odd powers of $Z$

does not disappear. As shown in appendix A (equation A.26), the correct asymptotic expansion of the leading term of the short time amplitude (equivalent of (1.23)) is given by

$$
\begin{aligned}
\frac{n}{(2 \pi \nu \tau)^{\frac{d}{2}}} \int_{x, z \in M} \mathfrak{g}^{\frac{1}{2}}(x) & \mathfrak{g}^{\frac{1}{2}}(z) \Delta(x \mid z) \exp \left(-\frac{1}{\nu \tau} \sigma(x \mid z)\right) \varphi(x) \psi(z)= \\
& =\varphi \cdot\left(\mathcal{G}+\sqrt{\tau}\left(-\frac{1}{n} \sqrt{\frac{\nu}{2 \pi}}\right) \mathcal{Q}-\tau \frac{\nu}{2} \stackrel{m}{\mathcal{L}}+\mathcal{O}\left(\tau^{\frac{3}{2}}\right)\right) \bullet \psi,
\end{aligned}
$$


where $\mathcal{Q}[\partial \Omega]$ is a delta bi-distribution localized on the boundary normalized to the boundary volume element $\mathfrak{q}^{\frac{1}{2}}$ understood as a distribution on spacetime,

$$
\varphi \cdot \mathcal{Q} \bullet \psi=\int_{\partial \Omega} \varphi \psi \mathfrak{q}^{\frac{1}{2}},
$$

and $\stackrel{\text { m }}{\mathcal{L}}$ is a particular ordering of the Laplace operator given by

$$
\begin{aligned}
& \stackrel{m}{\mathcal{L}}=\frac{1}{2}(\stackrel{\dddot{\mathcal{L}}}{\mathrm{L}}+\stackrel{\breve{\mathcal{L}}}{)}) \\
& \varphi \cdot \tilde{\mathcal{L}} \bullet \psi=-\int_{\Omega} \varphi\left(\nabla^{2} \psi\right) \mathfrak{g}^{\frac{1}{2}}
\end{aligned}
$$

Using this result it is easy to show that the expansion of the propagator $K_{\mathrm{o}}$ is

$$
\mathcal{G} \bullet K_{\mathrm{o}}(\tau) \bullet \mathcal{G}=\mathcal{G}+\sqrt{\tau}\left(-\frac{1}{n} \sqrt{\frac{\nu}{2 \pi}}\right) \mathcal{Q}-\tau \frac{\nu}{2} \stackrel{\mathfrak{F}}{\mathcal{F}}+\mathcal{O}\left(\tau^{\frac{3}{2}}\right)
$$

and $\stackrel{\mathfrak{F}}{\mathcal{F}}$ is a Laplace-like quadratic form with potential,

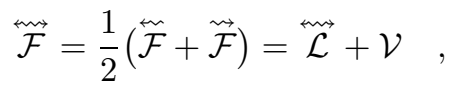

$$
\begin{aligned}
& \stackrel{\sim}{\mathcal{F}}^{\top}=\stackrel{\mathcal{F}}{=} \tilde{\mathcal{L}}+\mathcal{V} .
\end{aligned}
$$

The corrected potential $\mathcal{V}$ is given again by the expression $(1.27)$ and we have assumed that the condition (1.25) is satisfied.

We see that the expansion of the propagator has an additional term localized on the boundary $\partial \Omega$ proportional to $\sqrt{\tau}$. This $\tau$-dependence causes a problem because $\dot{K}_{\mathrm{o}}(0)$ is singular on the boundary. An origin of the singular term on the boundary lies in our careless approximation of the propagator by the short time amplitude (1.20). This approximation is correct only for endpoints sufficiently far from the boundary. For points near the boundary we have to investigate the structure of the propagator more thoroughly.

\section{Boundary correction term}

The short time amplitude (1.20) represents the dominant contribution to the heat kernel from trajectories near the geodesic joining endpoints $x_{\mathrm{f}}$ and $x_{\mathrm{i}}$. But in the case of a sum over trajectories restricted to the domain $\Omega$ there are other dominant terms given by contributions of trajectories near extremal paths which reflect on the boundary.

In general we should take into account trajectories with an arbitrary number of reflections on the boundary and compute the dominant contributions from all of them. However, for endpoints sufficiently far from the boundary the contributions from the reflected paths are negligible compared to the straight geodesic - for small $\tau$ only short paths contribute to the sum, and any trajectory with a reflection on the boundary is too long (see figure 2.1).

But for endpoints near to the boundary the contributions from the reflected trajectories can be comparable with the leading term. For the endpoints near a smooth boundary there exists exactly one extreme trajectory $\overline{\boldsymbol{x}}_{\mathrm{b}}(x \mid z)$ ) with one reflection which gives a contribution comparable to the contribution from the straight geodesic $\overline{\boldsymbol{x}}(x \mid z)$ ) (see figure 2.1).

The reflected extreme trajectory $\left.\overline{\boldsymbol{x}}_{\mathrm{b}}(x \mid z)\right)$ is an extremum of the leading term of the action (1.13) with the additional condition that the trajectory reflects on the boundary. Let's denote the point of 

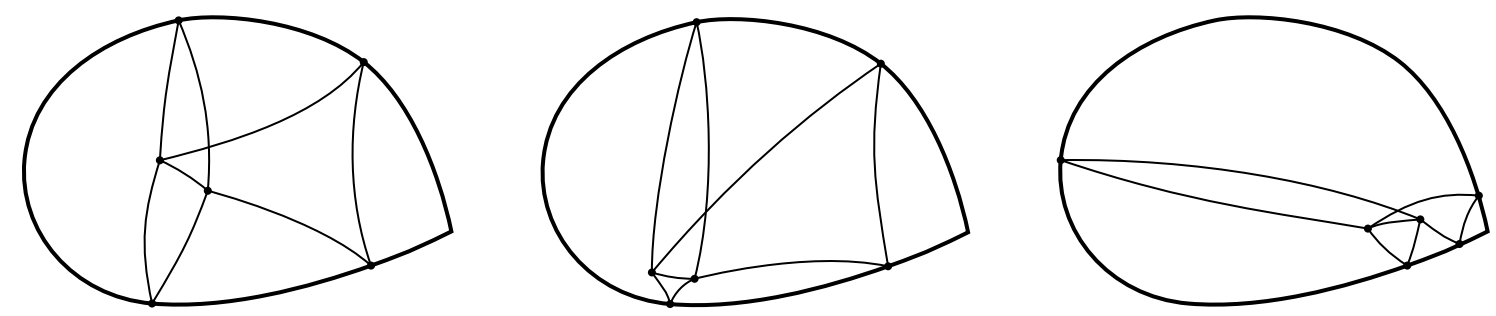

Figure 2.1: Example of extremal trajectories.

Dominant terms to sum over trajectories are given by trajectories near to extreme trajectories, possibly reflected from the boundary. If close endpoints are sufficiently far from the boundary, the reflected geodesics are longer then the straight geodesic. If the endpoints are near the boundary, there is a reflected geodesic with the length comparable to the length of straight one. Near the corner there are more reflected geodesics with comparable length.

the reflection $b(x \mid z)$ and the parameter for which the reflection occurs $\lambda_{\mathrm{r}}(x \mid z)$ and its complement $\lambda_{\mathrm{l}}(x \mid z)$. Clearly the trajectory is a joining of two geodesics

$$
\left[\tau, \overline{\boldsymbol{x}}_{\mathrm{b}}(x \mid z)\right]=\left[\lambda_{\mathrm{l}}(x \mid z) \tau, \overline{\boldsymbol{x}}(x \mid b(x \mid z))\right] \odot\left[\lambda_{\mathrm{r}}(x \mid z) \tau, \overline{\boldsymbol{x}}(b(x \mid z) \mid z)\right] .
$$

Using additivity of the action we get the value of its leading term

$$
\sigma_{\mathrm{b}}(x \mid z) \stackrel{\text { def }}{=} \nu I_{-1}\left(\overline{\boldsymbol{x}}_{\mathrm{b}}(x \mid z)\right)=\frac{\sigma_{\mathrm{l}}(x \mid z)}{\lambda_{\mathrm{l}}(x \mid z)}+\frac{\sigma_{\mathrm{r}}(x \mid z)}{\lambda_{\mathrm{r}}(x \mid z)},
$$

where, following the convention (B.77),

$$
\sigma_{1}(x \mid z)=\sigma(x \mid b(x \mid z)) \quad, \quad \sigma_{\mathrm{r}}(x \mid z)=\sigma(b(x \mid z) \mid z) \quad .
$$

The extremum requirement gives us conditions on $b$ and $\lambda_{\mathrm{l}}, \lambda_{\mathrm{l}}$,

$$
\begin{gathered}
\frac{\mathrm{D} \sigma(x \mid b(x \mid z))}{\lambda_{1}(x \mid z)}+\frac{\mathrm{D} \sigma(b(x \mid z) \mid z)}{\lambda_{\mathrm{r}}(x \mid z)}=0, \\
\frac{\sigma_{1}}{\lambda_{1}^{2}}=\frac{\sigma_{\mathrm{r}}}{\lambda_{\mathrm{r}}^{2}},
\end{gathered}
$$

where D denotes the orthogonal projection of the gradient on the boundary (the gradient with respect of the argument on the boundary). See appendix B for more details and other quantities defined on the boundary.

Now we can estimate the contribution from the trajectories near to the reflected geodesic $\overline{\boldsymbol{x}}_{\mathrm{b}}(x \mid z)$ ). Using reasoning similar to that used for deriving (1.20), we can write an approximation of the short time amplitude associated with the reflected geodesic as

$$
K_{\mathrm{b}}\left(\tau, x_{\mathrm{f}} \mid x_{\mathrm{i}}\right)=\frac{n}{(2 \pi \nu \tau)^{\frac{d}{2}}} \Delta_{\mathrm{b}}^{1-p}\left(x_{\mathrm{f}} \mid x_{\mathrm{i}}\right) \beta\left(\tau, x_{\mathrm{f}} \mid x_{\mathrm{i}}\right) \exp \left(-\frac{1}{\nu \tau} \sigma_{\mathrm{b}}\left(x_{\mathrm{f}} \mid x_{\mathrm{i}}\right)\right),
$$

where $\Delta_{\mathrm{b}}$ is Van Vleck-Morette determinant associated with the reflected geodesic (see $(\mathrm{B} .89)$ ). The coefficient $\beta$ is an analog of the coefficients $\alpha_{0}, \alpha_{1}$, only in this case we have to expect an expansion in powers of $\sqrt{\tau}$ :

$$
\beta(\tau, x \mid z)=\beta_{0}(x \mid z)+\sqrt{\tau} \beta_{\frac{1}{2}}(x \mid z)+\mathcal{O}(\tau)
$$


As we will see, the right normalization relative to the leading term $K_{\mathrm{o}}$ requires

$$
\beta_{0}(x \mid x)=1 .
$$

We did not bother to write down a potential term, because terms of order $\mathcal{O}(\tau)$ are negligible in the approximation we need, as can be seen in the calculation in appendix A. We also already anticipated an arbitrary power of the Van Vleck-Morette determinant, similarly to the choice (1.28).

Fixing this short time amplitude (i.e. specification of coefficients $p$ and $\beta$, or more precisely its coincidence limits as we will see below) together with amplitude (1.20) concludes the definition of the path integral in the domain with a smooth boundary.

\section{Short time behavior of the heat kernel}

Next we proceed to derive the short time behavior of the propagator. Again, the technical work is done in appendix A, where it is shown that for small $\tau$ we have the expansion (see eq. A.46)

$$
\begin{aligned}
\varphi \bullet \mathcal{G} \bullet K_{\mathrm{b}}(\tau) & \bullet \mathcal{G} \bullet \psi= \\
= & \sqrt{\tau} \frac{1}{n} \sqrt{\frac{\nu}{2 \pi}} \varphi \cdot \mathcal{Q} \bullet \psi- \\
& -\tau \frac{\nu}{2} \frac{1}{2} \varphi \bullet\left(\tilde{d \mathcal{F}}_{\tilde{n}}+\tilde{d \mathcal{F}}_{\tilde{n}}\right) \bullet \psi- \\
& -\tau \frac{\nu}{2} \varphi \cdot\left(\frac{1+p}{3} k+\beta \text {-terms }\right) \mathcal{Q} \bullet \psi+\mathcal{O}\left(\tau^{\frac{3}{2}}\right) .
\end{aligned}
$$

The $\beta$-terms contains coincidence limits of the first two derivatives of the coefficient $\beta$ on the boundary, and the exact form can be found in $(\mathrm{A.56}) . k$ is the trace of the external curvature $(\mathrm{B} .50)$ and $\tilde{d \mathcal{F}}_{\tilde{n}}, \tilde{d \mathcal{F}}_{\tilde{n}}$ are defined in (2.25). The normalization (2.15) ensures that the $\sqrt{\tau}$-term in $K_{\mathrm{b}}$ cancels exactly with such a term in $K_{\mathrm{o}}$.

So, if we add both dominant terms we get

$$
\begin{gathered}
K_{k}(\tau)=K_{\mathrm{o}}(\tau)+K_{\mathrm{b}}(\tau), \\
\mathcal{G} \bullet K_{\mathrm{k}}(\tau) \bullet \mathcal{G}=\mathcal{G}-\tau \frac{\nu}{2} \mathcal{F}_{\tilde{\mathrm{k}}}+\mathcal{O}\left(\tau^{\frac{3}{2}}\right),
\end{gathered}
$$

where $\mathcal{F}_{\tilde{k}}$ is the quadratic form of Laplace-like operator with the boundary conditions given by the choice of $\beta$ coefficients 6

$$
\begin{aligned}
& \mathcal{F}_{\tilde{k}}=\mathcal{F}_{\tilde{n}}-\Theta_{k} \quad, \\
& \mathcal{F}_{\tilde{n}}=\stackrel{\mathrm{d}}{\boldsymbol{\alpha}}_{\boldsymbol{\alpha}} \bullet\left(\chi g^{-1 \boldsymbol{\alpha} \boldsymbol{\beta}} \mathcal{G}\right) \cdot \widetilde{\mathrm{d}}_{\boldsymbol{\beta}}+(\chi \mathcal{V}), \\
& \Theta_{k} \stackrel{\text { def }}{=}-\left(\frac{1+p}{3} k+\beta \text {-terms }\right) \mathcal{Q},
\end{aligned}
$$

where $\chi$ is the characteristic function of the domain $\Omega$. Here $\mathcal{F}_{\tilde{n}}$ is the standard quadratic form which appears in the action for non-interacting scalar field with a potential evaluated on the domain $\Omega$ and $\Theta_{k}$ is a bi-distribution localized on the boundary.

The short time behavior (2.18) together with the composition law again prove the heat equation for the propagator:

$$
-\mathcal{G} \bullet \dot{K}_{k}(\tau)=\frac{\nu}{2} \mathcal{F}_{\tilde{k}} \bullet K_{k}(\tau)
$$

The quadratic form $\mathcal{F}_{\tilde{k}}$ identifies what kind of boundary conditions the propagator $K_{\mathrm{k}}$ satisfies. If we compare $\mathcal{F}_{\tilde{k}}$ with the operator $\widetilde{\mathcal{F}}$ (all derivatives act to the right) we find

$$
\begin{aligned}
\mathcal{F}_{\tilde{k}} & =\breve{\mathcal{F}}+\widetilde{d \mathcal{F}}_{\tilde{k}}, \\
\tilde{d \mathcal{F}}_{\tilde{k}} & =\widetilde{d \mathcal{F}}_{\tilde{n}}-\Theta_{k},
\end{aligned}
$$


where the bi-distribution $\mathfrak{d \mathcal { F }}_{\tilde{n}}$ is essentially integration of a value of the left argument and a momenta of the right argument over boundary of the domain

$$
\varphi \cdot \widetilde{d \mathcal{F}}_{\tilde{n}} \bullet \psi=\int_{\partial \Omega} \varphi \vec{n}^{\alpha} \mathrm{d}_{\boldsymbol{\alpha}} \psi \mathfrak{q}^{\frac{1}{2}} .
$$

To see clearly what kind of boundary conditions the propagator satisfies, we write the heat equation in the following way

$$
-\mathcal{G} \bullet \dot{K}_{k}(\tau)=\frac{\nu}{2} \tilde{\mathcal{F}}_{\mathfrak{F}} K_{k}(\tau)+\frac{\nu}{2} \tilde{d \mathcal{F}}_{\tilde{k}} \bullet K_{k}(\tau)
$$

The solution of the heat equation is smooth for non-zero time $\tau$. Therefore the left-hand side is smooth, as well as the first term on the right-hand side. The second term is localized on the boundary and therefore it has to vanish. So we find

$$
\widetilde{d \mathcal{F}}_{\tilde{\mathrm{k}}} \bullet K_{\mathrm{k}}(\tau)=0
$$

Or, if we define maps $\underline{\varphi}$ and $\underline{\pi}$ which assign a value $\varphi=\underline{\varphi} \bullet \phi$ and a momenta $\pi=\underline{\pi} \bullet \phi$ on the boundary to a spacetime function $\phi$, we can writel

$$
\begin{gathered}
\widetilde{d \mathcal{F}}_{\tilde{n}}=\varphi \cdot \underline{\pi}, \\
\Theta_{k}=-\varphi \cdot\left(\left(\frac{1+p}{3} k+\beta \text {-terms }\right) \mathfrak{q}^{\frac{1}{2}} \delta\right) \cdot \underline{\varphi}
\end{gathered}
$$

and the boundary conditions get the form

$$
\left(\underline{\pi}+\left(\frac{1+p}{3} k+\beta \text {-terms }\right) \underline{\varphi}\right) \bullet K_{k}(\tau)=0 .
$$

We see that $K_{k}$ satisfies Robin-like boundary conditions.

Let us summarize. We have found that the propagator given by the sum of amplitudes over histories in the domain $\Omega$ with fixed endpoints and inner time $\tau$ is a solution of the heat equation with specific boundary conditions. The boundary conditions depend on the definition of the path integral through the coincidence limits of derivatives of coefficients $\beta$ in the short time amplitude (2.13). In general, they are Robin-like conditions with a non-degenerate coefficient in front of momentum.

\section{Green function}

In the case of a relativistic particle the inner time is an unphysical quantity, and all physically distinguishable sets of histories should contain histories with all possible inner times. Therefore we will compute the amplitude associated with the set of histories with fixed endpoints but withouta restriction on the inner time. As before, we will call this amplitude the Feynman Green function 3

$$
\frac{1}{n} G_{k}^{F}(x \mid z)=\int_{\tau \in \mathbb{R}^{+}} \frac{1}{n} K_{k}(\tau, x \mid z) \frac{\nu}{2} d \tau .
$$

Using the heat equation and initial conditions for the heat kernel, the integration gives

$$
\mathcal{F}_{\tilde{k}} \bullet G_{k}^{F}=\delta .
$$

So, $G_{k}^{F}$ is the inverse of $\mathcal{F}_{\tilde{k}}$ and restricted to smooth sources it satisfies the same boundary conditions as the propagator $K_{\mathrm{k}}$. 


\section{Amplitude of particles emitted by a source}

Let us compute the amplitude $Z_{\mathrm{k}}^{(1)}(J)$ of a set of histories which end at a given point $x$ and are emitted by a source described by a spacetime dependent amplituded $n J$. We will call it the oneparticle amplitude. It is clearly given by

$$
Z_{\mathrm{k}}^{(1)}(J)=G_{\mathrm{k}}^{F} \bullet J=\bar{\phi}_{\mathrm{k}}(J)
$$

It satisfies the same boundary conditions as the Feynman Green function.

We will interpret the boundary conditions as a consequence of the fact that we have not allowed particles to start on the boundary. More precisely, we have allowed the smooth source to be non-zero up to the boundary, but we have not allowed an emission of particles from the boundary comparable to an emission from a finite volume.

We can ask why some particular boundary conditions means that no particles are emitted from the boundary. What about different boundary conditions? Why is the choice of the conditions above special? We are touching a question of what kind of particles we are dealing with. What does it mean that no particles are emitted (or absorbed - for scalar particle the meanings are interchangeable if we do not distinguish initial and final parts of the boundary).

First we have to realize that the statement "no particles on the boundary" has to be interpreted as a result of a measurement on the boundary. We have to arrange apparatuses on the whole boundary which are sensitive to particles, and when all these devices measure no particle we can speak about no emission or absorption. Clearly this is very complicated global measurement. It depends on an exact arrangement of experimental devices on the whole boundary and on an interaction of particles with devices. We have hidden this dependence in the definition of the path integral through the non-specified $\beta$-terms. Therefore we see that we cannot expect a unique canonical meaning for the statement "no particles on the boundary". Only if we specify the kind of measurement we are performing we do have a meaning for this statement. And necessary information about experimental devices can be phenomenologically characterized by the choice of boundary conditions of the type we encountered above.

In the following paper [9] we will discuss what interaction of particles with boundary leads to different boundary conditions.

So, the different boundary conditions correspond to different type of detections of particles on the boundary and in the following paper we will see that it can correspond to different definitions of vacua in the standard particle theory - in the scalar field theory in curved spacetime.

\section{Emission from the boundary}

Of course, we can ask what is the amplitude to find a particle at a point $x$ if we allow an emission from the boundary. Let us assume that the amplitude of the emission from the boundary is given by a density $n j$ on the boundary manifold, which we call the boundary source. The amplitude $Z_{\mathrm{k}}^{(1)}(\tau ; j)$ associated with the set of one-particle histories which are emitted by this boundary source and end in time $\tau$ at a point $x$, can be written using the boundary propagator $K_{\mathrm{k}}^{-}$

$$
Z_{\mathrm{k}}^{(1)}(\tau ; j)=K_{\mathrm{k}}^{-1}(\tau) \cdot j
$$

The boundary propagator propagates between points inside of the domain and boundary sources. It has the character of a function on the domain $\Omega$ in the left argument and the function on the boundary manifold $\partial \Omega$ in the right argument.

Clearly, the boundary propagator satisfies a composition law similar to (1.7)

$$
K_{\mathrm{k}}^{\dashv}(\tau)=K_{\mathrm{k}}(\tau-\epsilon) \cdot \mathcal{G} \bullet K_{\mathrm{k}}^{-1}(\epsilon)
$$


We can take a limit $\epsilon \rightarrow 0$ and get

$$
\begin{gathered}
K_{\mathrm{k}}^{\dashv}(\tau)=K_{\mathrm{k}}(\tau) \bullet \stackrel{\leftrightarrow}{K}_{\mathrm{k}}, \\
\stackrel{\mathrm{K}}{\mathrm{k}}_{\mathrm{k}} \stackrel{\text { def }}{=} \mathcal{G} \bullet K_{\mathrm{k}}^{-\dashv}(0) .
\end{gathered}
$$

We see that the amplitude is given by the propagator $K_{k}(\tau)$ with no emission from the boundary, and by the boundary term $\stackrel{\aleph}{K}_{k}$ which "translates" between the space of sources on the boundary and amplitudes in the domain. Similarly, if we sum over all possible inner times we get

$$
Z_{\mathrm{k}}^{(1)}(j)=G_{\mathrm{k}}^{F} \bullet \stackrel{\leftrightarrow}{K}_{k} \cdot j
$$

The boundary term $\stackrel{\leftrightarrow}{K}_{k}$ is a zero-time amplitude, so it is straightforward to estimate it. The short time amplitude approximation similar to (1.20) for the boundary propagator is

$$
\begin{aligned}
& \phi \bullet \mathcal{G} \bullet K_{k}^{-1}(\tau) \cdot j= \\
& \quad=\frac{n}{(2 \pi \nu \tau)^{\frac{d}{2}}} \int_{\substack{x \in \Omega \\
\hat{y} \in \partial \Omega}} \mathfrak{g}^{\frac{1}{2}}(x) \phi(x) j(\widehat{y}) \Delta(x \mid \widehat{y}) \exp \left(-\frac{1}{\nu \tau} \sigma(x \mid \widehat{y})\right)(1+\mathcal{O}(\sqrt{\tau}))= \\
& \quad=\int_{\widehat{y} \in \partial \Omega} \phi(\widehat{y}) j(\widehat{y})(1+\mathcal{O}(\sqrt{\tau}))=\phi \bullet \underline{\varphi} \cdot j(1+\mathcal{O}(\sqrt{\tau})) .
\end{aligned}
$$

Therefore, for zero inner time we get

$$
\stackrel{\dddot{K}}{k}_{\mathrm{k}}=\varphi
$$

It means that the emission from the boundary is equivalent to the emission of particles inside of the domain but with a distributional source $\partial J=j \cdot \underline{\varphi}$ with support on the boundary

Allowing both boundary sources and sources inside of the domain, we find that the one-particle amplitude is

$$
Z_{\mathrm{k}}^{(1)}(J, \partial J)=G_{\mathrm{k}}^{F} \bullet(J+\partial J)=\bar{\phi}_{\mathrm{k}}(J+\partial J)
$$

A careful discussion of the distributional character of introduced boundary sources, differential operators and Green functions shows (see [13]) that it satisfies the equation of motion in the expected form

$$
\mathcal{F}_{\tilde{k}} \bullet \bar{\phi}_{\mathrm{k}}(J+\partial J)=J+\partial J
$$

with boundary conditions fixed by the boundary source

$$
\partial J=\widetilde{d \mathcal{F}}_{\tilde{k}} \bullet \bar{\phi}_{k}(J+\partial J)
$$




\section{Conclusion}

In this paper we studied sum-over-histories quantization of relativistic particle on a bounded domain of the spacetime. We showed that we have to modify a definition of the path integral by adding terms corresponding to paths reflected on the boundary of the domain. Such contributions can be dominant in the short time approximation near the boundary in addition to the usual dominant contributions from the straight geodesic. They compensate other terms localized on the boundary which arise from restriction of non-reflected paths to the domain. They also specify the exact form of the boundary conditions for the propagator and Green functions. We found that boundary conditions have Robin-like form and their exact form depends on the details of the definition of the path integral - a non-uniqueness is hidden in the specification of $\beta$-coefficients in the short time amplitude (2.13).

We interpreted the specific boundary conditions as a consequence of an interaction with apparatuses localized on the boundary which allow us to define a notion of particles. Because of the non-uniqueness of the definition of the path integral we do not have a uniqueness in the definition of particles. In this correspondence the boundary condition can be also viewed as a phenomenological description of the specific kind of particles.

In the next paper 9 we will discuss a freedom in boundary conditions in more detail. We will show that inclusion of some additional interaction with measurement apparatuses on the boundary can lead to general boundary conditions which include the conditions corresponding to a definition of particles in the quantum scalar field theory. 


\section{A Asymptotic expansion of the leading term in the heat kernel}

\section{Vector space}

In a vector space $V$ equipped with a positive nondegenerate quadratic form $g$ a simple Gaussian integration gives

$$
\begin{aligned}
& \frac{1}{(2 \pi \nu \tau)^{\frac{d}{2}}} \int_{X, Z \in V} \mathfrak{g}^{\frac{1}{2}}(X) \mathfrak{g}^{\frac{1}{2}}(Z) \varphi(X) \psi(Z) \exp \left(-\frac{1}{2 \nu \tau}(X-Z) \cdot g \cdot(X-Z)\right)= \\
& =\frac{1}{(2 \pi \nu)^{\frac{d}{2}}} \int_{X \in V} \mathfrak{g}^{\frac{1}{2}}(X) \varphi(X) \int_{Y \in V} \mathfrak{g}^{\frac{1}{2}}(Y) \psi(X+\sqrt{\tau} Y) \exp \left(-\frac{1}{2 \nu} Y \cdot g \cdot Y\right)= \\
& =\frac{1}{(2 \pi \nu)^{\frac{d}{2}}} \int_{X \in V} \mathfrak{g}^{\frac{1}{2}}(X) \varphi(X) \sum_{k \in \mathbb{N}_{0}} \tau^{\frac{k}{2}}\left[\partial_{\boldsymbol{\alpha}_{1}} \ldots \partial_{\boldsymbol{\alpha}_{\boldsymbol{k}}} \psi\right](X) \times \\
& \quad \times \frac{1}{k !} \int_{Y \in V} \mathfrak{g}^{\frac{1}{2}}(Y) Y^{\boldsymbol{\alpha}_{1}} \ldots Y^{\boldsymbol{\alpha}_{\boldsymbol{k}}} \exp \left(-\frac{1}{2 \nu} Y \cdot g \cdot Y\right)= \\
& =\int_{X \in V} \mathfrak{g}^{\frac{1}{2}}(X) \varphi(X) \sum_{m \in \mathbb{N}_{0}} \frac{(2 m-1) !}{(2 m) !}(\tau \nu)^{m}\left[\left(g^{-1 \boldsymbol{\alpha} \boldsymbol{\beta}} \partial_{\boldsymbol{\alpha}} \partial_{\boldsymbol{\beta}}\right)^{m} \psi\right](X)= \\
& =\sum_{m \in \mathbb{N}_{0}} \frac{1}{m !}\left(-\frac{\nu \tau}{2}\right)^{m} \varphi \cdot \mathcal{L}^{m} \bullet \psi .
\end{aligned}
$$

Here $\varphi, \psi$ are test functions, $\mathfrak{g}^{\frac{1}{2}}$ is the constant volume element of the metric $g, \nu$ is a number $\left(\operatorname{Re} \frac{1}{\nu} \geqslant 0\right)$ and $\mathcal{L}^{m}$ represents a bi-distribution

$$
\varphi \cdot \mathcal{L}^{m} \bullet \psi=\int_{V} \mathfrak{g}^{\frac{1}{2}} \varphi\left[\left(g^{-1 \boldsymbol{\alpha} \boldsymbol{\beta}} \partial_{\boldsymbol{\alpha}} \partial_{\boldsymbol{\beta}}\right)^{m} \psi\right]=\int_{V} \mathfrak{g}^{\frac{1}{2}} \psi\left[\left(g^{-1 \boldsymbol{\alpha} \boldsymbol{\beta}} \partial_{\boldsymbol{\alpha}} \partial_{\boldsymbol{\beta}}\right)^{m} \varphi\right] .
$$

It can be viewed as an " $m$-th" power of the Laplace quadratic form $\mathcal{L}$ associated with the metric $g$ on the vector space $V$.

So we can write the asymptotic expansion for small $\tau$ as

$$
\frac{n}{(2 \pi \nu \tau)^{\frac{d}{2}}} \mathfrak{g}^{\frac{1}{2}}(X) \mathfrak{g}^{\frac{1}{2}}(Z) \exp \left(-\frac{1}{2 \nu \tau}(X-Z) \cdot g \cdot(X-Z)\right)=\sum_{m \in \mathbb{N}_{0}} \frac{1}{m !}\left(-\frac{\tau \nu}{2}\right)^{m} \mathcal{L}^{m} .
$$

Here we allowed the metric $g$ to be Lorentzian - this case can be obtained by analytical continuation in the phase factor $n$ which characterizes the signature of the metric.

In the case of the vector space the expression on the left side of eq. A.3 is the heat kernel of the operator $\mathcal{L}$. Because the right side is formally the exponential we see that the expansion is

exact. To say more about convergence it is necessary to specify functional spaces on which all the operators act and we will not do this here. But see e.g. [14] for some details. 


\section{Manifold without a boundary}

Now we would like to find the expansion of the similar expression in a general manifold $M$ without boundary. More precisely, we want to expand

$$
\frac{n}{(2 \pi \nu \tau)^{\frac{d}{2}}} \Delta(x \mid z) \mathfrak{g}^{\frac{1}{2}}(x) \mathfrak{g}^{\frac{1}{2}}(z) \exp \left(-\frac{1}{\nu \tau} \sigma(x \mid z)\right)
$$

for small $\tau$.

We smooth both arguments with test functions $\varphi, \psi$ and note that for a small $\tau$ the integration over $x$ and $z$ is dominated by a diagonal $x \approx z$ thanks to $\sigma(x \mid z) \approx 0$ for $x \approx z$. Therefore for a fixed $x$ we can restrict integration over $z$ to a normal neighborhood of $x$. In this neighborhood we can change variables $z \rightarrow Z$ with

$$
z=\boldsymbol{u}_{x}(\sqrt{\tau} Z) \approx x+\sqrt{\tau} Z
$$

where $\boldsymbol{u}_{x}(\epsilon Z)$ is a geodesic with an origin $x$ and initial tangent vector $Z$ (see (B.1)). This is the exact meaning of "adding" of a vector to a point as mentioned after equation (1.16).

The Jacobian associated with this change of variables is given by Van-Vleck Morette determinant $(\operatorname{see}(B .43))$

$$
\left(\boldsymbol{u}_{x}^{-1 \star} \mathfrak{g}^{\frac{1}{2}}\right)(\sqrt{\tau} Z)=\tau^{\frac{d}{2}} \mathfrak{g}^{\frac{1}{2}}(x)[Z] \Delta^{-1}(x \mid z),
$$

where $\mathfrak{g}^{\frac{1}{2}}(x)[Z]$ is understood as a constant measure on the target vector space $\mathbf{T}_{x} M$. After a change of variables, using (B.16), expanding $\psi$ and performing a Gaussian integration, we get

$$
\begin{aligned}
& \frac{n}{(2 \pi \nu \tau)^{\frac{d}{2}}} \int_{x, z \in M} \Delta(x \mid z) \mathfrak{g}^{\frac{1}{2}}(x) \mathfrak{g}^{\frac{1}{2}}(z) \varphi(x) \psi(z) \exp \left(-\frac{1}{\nu \tau} \sigma(x \mid z)\right)= \\
& =\frac{n}{(2 \pi \nu \tau)^{\frac{d}{2}}} \int_{x \in M} \mathfrak{g}^{\frac{1}{2}}(x) \varphi(x) \int_{Z \in \mathbf{T}_{x} M} \mathfrak{g}^{\frac{1}{2}}(x)[Z] \times \\
& \quad \times\left(\sum_{k \in \mathbb{N}_{0}} \frac{1}{k !} \tau^{\frac{k}{2}} \psi_{k \boldsymbol{\alpha}_{1} \ldots \boldsymbol{\alpha}_{\boldsymbol{k}}}(x) Z^{\boldsymbol{\alpha}_{1}} \ldots Z^{\boldsymbol{\alpha}_{k}}\right) \exp \left(-\frac{1}{\nu \tau} Z \cdot g(x) \cdot Z\right)= \\
& =\int_{M} \mathfrak{g}^{\frac{1}{2}} \varphi\left(\psi+\frac{\nu \tau}{2} g^{-1 \boldsymbol{\alpha} \boldsymbol{\beta}} \psi_{2} \boldsymbol{\alpha} \boldsymbol{\beta}+\mathcal{O}\left(\tau^{2}\right)\right) .
\end{aligned}
$$

Using (B.27) we have $\psi_{2}=\nabla \mathrm{d} \psi$, so we get

$$
\frac{n}{(2 \pi \nu \tau)^{\frac{d}{2}}} \Delta(x \mid z) \mathfrak{g}^{\frac{1}{2}}(x) \mathfrak{g}^{\frac{1}{2}}(z) \exp \left(-\frac{1}{\nu \tau} \sigma(x \mid z)\right)=\mathcal{G}-\frac{\nu \tau}{2} \mathcal{L}+\mathcal{O}\left(\tau^{2}\right)
$$

\section{Half line}

Next we will investigate the simplest case of the manifold with the boundary — half line $\mathbb{R}^{+}$. We assume it is equipped with a special coordinate $\eta$ which selects a measure and derivative

$$
\mu=d \eta \quad, \quad \mathcal{M}=\mu \delta \quad, \quad \partial=\frac{\partial}{\partial \eta} .
$$

We define bi-distributions of the $m$-th derivative

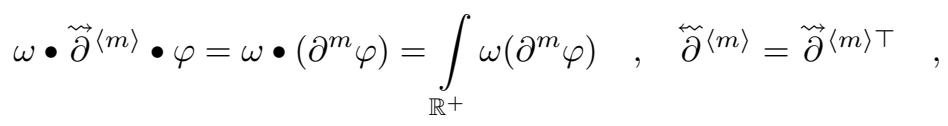


and

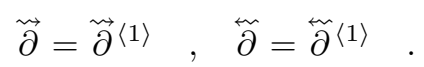

We can define also a boundary delta bi-distribution $\mathcal{D}$ as (the boundary is one point now)

$$
\varphi \bullet \mathcal{D} \bullet \psi=\left.(\varphi \psi)\right|_{\text {boundary }} .
$$

Integration by parts can be expressed by the relation

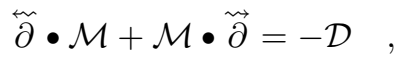

$$
\begin{aligned}
& \tilde{\partial}^{\langle m+1\rangle} \bullet \mathcal{M}+(-1)^{m} \mathcal{M} \bullet \dddot{\partial}^{\langle m+1\rangle}=-\sum_{k=0, \ldots, m} \dddot{\partial}^{\langle m-k\rangle} \bullet \mathcal{D} \cdot \dddot{\partial}^{\langle k\rangle} \text {. }
\end{aligned}
$$

Next we define quadratic forms of powers of the Laplace operator

$$
\begin{aligned}
& \ddot{\mathcal{L}}=-\mathcal{M} \bullet \dddot{\partial}^{\langle 2\rangle} \quad, \quad \tilde{\mathcal{L}}^{\langle m\rangle}=(-1)^{m} \mathcal{M} \bullet \tilde{\partial}^{\langle 2 m\rangle},
\end{aligned}
$$

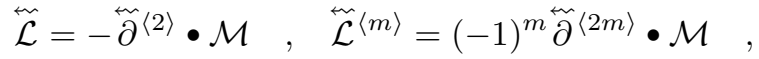

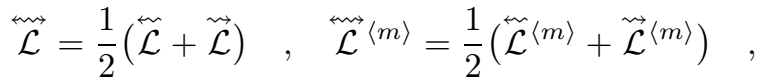

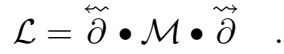

The symplectic form on the boundary is

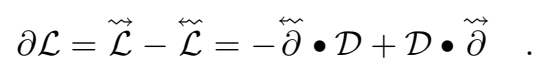

It is straightforward to check that

$$
\widetilde{\mathcal{L}}^{\langle m\rangle}-\stackrel{\tilde{\mathcal{L}}}{ }^{\langle m\rangle}=(-1)^{m} \sum_{\substack{k, l \in \mathbb{N}_{0} \\ k+l+1=2 m}} \tilde{\partial}^{\langle k\rangle} \bullet \mathcal{D} \bullet \stackrel{\partial}{\partial}^{\langle l\rangle} .
$$

Now we prove the following expansion for small $\tau$

$$
\begin{aligned}
& \frac{1}{\sqrt{2 \pi \tau \nu}} \exp \left(-\frac{1}{2 \tau \nu}(\xi-\zeta)^{2}\right) \mu(\xi) \mu(\zeta)= \\
& =\sum_{m \in \mathbb{N}_{0}} \frac{1}{\Gamma\left(\frac{m}{2}+1\right)}\left(\frac{\tau \nu}{2}\right)^{\frac{m}{2}} \frac{1}{2}\left(\stackrel{\tilde{\partial}}{\partial}^{\langle m\rangle} \bullet \mathcal{M}+\mathcal{M} \cdot \stackrel{\check{\partial}}{\partial}^{\langle m\rangle}\right)= \\
& =\sum_{m \in \mathbb{N}_{0}} \frac{(-1)^{m}}{m !}\left(\frac{\tau \nu}{2}\right)^{m} \stackrel{m}{\mathcal{L}}\langle m\rangle+
\end{aligned}
$$

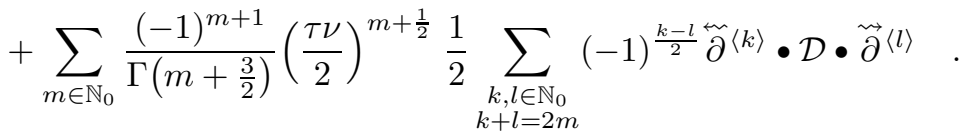

Here $\nu$ is a complex number such that $\frac{1}{\nu}$ has non-negative real part. Strictly speaking, the following derivation needs a positive real part; but for an imaginary value of $\nu$ the relation can be obtained by limiting procedure. Because only a combination the $\tau \nu$ appears in the equation, we drop $\nu$ in the following derivation - it can be easily restored by inspection of the $\tau$-dependence. 
Clearly, the second equality follows from integration by parts (A.13). To prove the first one we smooth it with test functions $\varphi$ and $\psi$ and get

$$
\begin{aligned}
& \frac{1}{\sqrt{2 \pi \tau}} \int_{\xi, \zeta \in \mathbb{R}^{+}} \exp \left(-\frac{1}{2 \tau}(\xi-\zeta)^{2}\right) \varphi(\xi) \psi(\zeta) \mu(\xi) \mu(\zeta)= \\
& =\frac{1}{\sqrt{2 \pi \tau}} \int_{\xi \in\langle 0, \epsilon\rangle} d \xi \int_{\zeta \in \mathbb{R}^{+}} d \zeta \varphi(\xi) \psi(\zeta) \exp \left(-\frac{1}{2 \tau}(\xi-\zeta)^{2}\right)+ \\
& \quad+\frac{1}{\sqrt{2 \pi \tau}} \int_{\xi \in\langle\epsilon, \infty\rangle} d \xi \int_{\zeta \in \mathbb{R}^{+}} d \zeta \varphi(\xi) \psi(\zeta) \exp \left(-\frac{1}{2 \tau}(\xi-\zeta)^{2}\right)
\end{aligned}
$$

for some $\epsilon \in \mathbb{R}^{+}$.

For a small $\tau$ the exponential suppresses any contribution except from $\xi \approx \zeta$. Therefore for small $\epsilon$ only small values of $\xi$ and $\zeta$ contribute to the first term of the last equation. We can rescale variables by factor $\sqrt{\tau}$ and expand $\varphi$ and $\psi$ at zero and obtain

$$
\begin{aligned}
& \frac{1}{\sqrt{2 \pi \tau}} \int_{\xi \in\langle 0, \epsilon\rangle} d \xi \int_{\zeta \in \mathbb{R}^{+}} d \zeta \varphi(\xi) \psi(\zeta) \exp \left(-\frac{1}{2 \tau}(\xi-\zeta)^{2}\right)= \\
& =\frac{\tau}{\sqrt{2 \pi \tau}} \int_{\xi \in\left\langle 0, \frac{\epsilon}{\sqrt{\tau}}\right\rangle} d \xi \varphi(\sqrt{\tau} \xi) \sum_{l \in \mathbb{N}_{0}} \tau^{\frac{l}{2}} \psi^{\langle l\rangle}(0) \frac{1}{l !} \int_{l \in \mathbb{R}^{+}} d \zeta \zeta^{l} \exp \left(-\frac{1}{2}(\xi-\zeta)^{2}\right)= \\
& =\frac{1}{\sqrt{2 \pi}} \sum_{k, l \in \mathbb{N}_{0}} \tau^{\frac{k+l+1}{2}} \varphi^{\langle k\rangle}(0) \psi^{\langle l\rangle}(0) \frac{1}{k !} \int_{\xi \in\left\langle 0, \frac{\epsilon}{\sqrt{\tau}}\right\rangle} d \xi \xi^{k} \mathrm{R}_{l+1}(\xi)
\end{aligned}
$$

where we have used the definition of special functions $\mathrm{R}_{l}$ (C.1). Properties of these special functions are summarized in appendix C. Using equation (C.17) the last expression gives

$$
\begin{aligned}
& \frac{1}{\sqrt{2 \pi}} \sum_{k, l \in \mathbb{N}_{0}} \tau^{\frac{k+l+1}{2}} \varphi^{\langle k\rangle}(0) \psi^{\langle l\rangle}(0) \times \\
& \times\left((-1)^{l+1} \mathrm{R}_{k+l+2}(0)+\right. \\
& \quad+\frac{\sqrt{2 \pi}}{l ! k !} \sum_{\substack{m \in \mathbb{N}_{0} \\
2 m \leqslant l}} \frac{(2 m-1) ! !}{k+l-2 m+1}\left(\begin{array}{c}
l \\
2 m
\end{array}\right)\left(\frac{\epsilon}{\sqrt{\tau}}\right)^{k+l-2 m+1}+ \\
& \left.\quad+(-1)^{l} \sum_{m=0, \ldots, k} \frac{1}{m !}\left(\frac{\epsilon}{\sqrt{\tau}}\right)^{m} \mathrm{R}_{k+l-m+2}\left(-\frac{\epsilon}{\sqrt{\tau}}\right)\right)= \\
& =\sum_{m \in \mathbb{N}_{0}} \tau^{\frac{m+1}{2}}\left(2^{\frac{m+1}{2}} \Gamma\left(\frac{m+1}{2}+1\right)\right)^{-1} \frac{1}{2} \sum_{l=0, \ldots, m}(-1)^{l+1} \varphi^{\langle m-l\rangle}(0) \psi^{\langle l\rangle}(0)+ \\
& \quad+\sum_{m \in \mathbb{N}_{0}} \frac{\tau^{m}}{(2 m) ! !} \sum_{k, l \in \mathbb{N}_{0}} \frac{\epsilon^{k+l+1}}{k ! l !(k+l+1)} \varphi^{\langle k\rangle}(0) \psi^{\langle l+2 m\rangle}(0)+ \\
& \quad+\exp \left(-\frac{1}{2} \frac{\epsilon^{2}}{\tau}\right) \mathcal{O}(\tau)=
\end{aligned}
$$




$$
\begin{aligned}
=- & \sum_{m \in \mathbb{N}} \frac{\tau^{m}}{(2 m) ! !} \frac{1}{2} \sum_{l=0, \ldots, 2 m-1}(-1)^{l} \varphi^{\langle 2 m-l-1\rangle}(0) \psi^{\langle l\rangle}(0)+ \\
& +\sum_{m \in \mathbb{N}_{0}} \frac{\tau^{m+\frac{1}{2}}}{(2 m+1) ! !} \frac{-1}{\sqrt{2 \pi}} \sum_{l=0, \ldots, 2 m}(-1)^{l} \varphi^{\langle 2 m-l\rangle}(0) \psi^{\langle l\rangle}(0)+ \\
& +\sum_{m \in \mathbb{N}_{0}} \frac{\tau^{m}}{(2 m) ! !} \sum_{k, l \in \mathbb{N}_{0}} \frac{\epsilon^{k+l+1}}{k ! l !(k+l+1)} \varphi^{\langle k\rangle}(0) \psi^{\langle l+2 m\rangle}(0)+ \\
& +\exp \left(-\frac{1}{2} \frac{\epsilon^{2}}{\tau}\right) \mathcal{O}(\tau) .
\end{aligned}
$$

Here we have used relations $(\overline{C .10})$ and $(\mathrm{C.14})$.

In the second term of the expression A.18) we change variables $\zeta \rightarrow \eta=\frac{1}{\sqrt{\tau}}(\xi-\zeta)$ using again the fact that only the contribution from $\zeta \approx \xi$ is not suppressed by the exponential. Next we expand $\psi$ around $\eta=0$ and obtain

$$
\begin{aligned}
\frac{1}{\sqrt{2 \pi \tau}} & \int_{\xi \in\langle\epsilon, \infty\rangle} d \xi \int_{\zeta \in \mathbb{R}^{+}} d \zeta \varphi(\xi) \psi(\zeta) \exp \left(-\frac{1}{2 \tau}(\xi-\zeta)^{2}\right)= \\
= & \frac{1}{\sqrt{2 \pi \tau}} \int_{\xi \in\langle\epsilon, \infty\rangle} d \xi \varphi(\xi) \sum_{k \in \mathbb{N}_{0}} \psi^{\langle k\rangle}(\xi) \tau^{\frac{k}{2}} \times \\
& \times\left(\frac{1}{k !} \int_{\eta \in \mathbb{R}} d \eta \eta^{k} \exp \left(-\frac{1}{2} \eta^{2}\right)-\frac{1}{k !} \int_{\eta \in\left\langle-\infty, \frac{\xi}{\sqrt{\tau}}\right\rangle} d \eta \eta^{k} \exp \left(-\frac{1}{2} \eta^{2}\right)\right)= \\
= & \sum_{m=\mathbb{N}_{0}} \frac{\tau^{m}}{(2 m) ! !} \int_{\xi \in\langle\epsilon, \infty\rangle} d \xi \varphi(\xi) \psi^{\langle 2 m\rangle}(\xi)- \\
& -\frac{1}{\sqrt{2 \pi \tau}} \sum_{k \in \mathbb{N}_{0}} \tau^{\frac{k}{2}} \sum_{l=0, \ldots, k} \frac{(-1)^{k}}{l !} \int_{\langle\epsilon, \infty\rangle} d \xi\left(\frac{\xi}{\sqrt{\tau}}\right)^{l} \mathrm{R}_{k-l+1}\left(-\frac{\xi}{\sqrt{\tau}}\right) \varphi(\xi) \psi^{\langle k\rangle}(\xi)= \\
= & \sum_{m=\mathbb{N}_{0}} \frac{\tau^{m}}{(2 m) ! !} \int_{\xi \in \mathbb{R}^{+}} d \xi \varphi(\xi) \psi^{\langle 2 m\rangle}(\xi)-\sum_{m=\mathbb{N}_{0}} \frac{\tau^{m}}{(2 m) ! !} \int_{\xi \in\langle 0, \epsilon\rangle} d \xi \varphi(\xi) \psi^{\langle 2 m\rangle}(\xi)+ \\
& +\exp \left(-\frac{1}{2} \frac{\epsilon^{2}}{\tau}\right) \mathcal{O}(\tau) .
\end{aligned}
$$

Here we used $(\overline{\mathrm{C} .15}),(\overline{\mathrm{C} .11}),(\overline{\mathrm{C} .16})$ and $(\overline{\mathrm{C} .14})$. For small $\epsilon$ we can expand $\varphi$ and $\psi^{\langle 2 m\rangle}$ about zero in the second term of the last expression. Performing also an integration by parts in the first term transforms the last expression to

$$
\begin{aligned}
& \sum_{m \in \mathbb{N}_{0}} \frac{\tau^{m}}{(2 m) ! !} \frac{1}{2} \int_{\xi \in \mathbb{R}^{+}} d \xi\left(\varphi^{\langle 2 m\rangle}(\xi) \psi(\xi)+\varphi(\xi) \psi^{\langle 2 m\rangle}(\xi)\right)+ \\
& \quad+\sum_{m \in \mathbb{N}} \frac{\tau^{m}}{(2 m) ! !} \frac{1}{2} \sum_{l=0, \ldots, 2 m-1}(-1)^{l} \varphi^{\langle 2 m-l-1\rangle}(0) \psi^{\langle l\rangle}(0)- \\
& \quad-\sum_{m \in \mathbb{N}_{0}} \frac{\tau^{m}}{(2 m) ! !} \sum_{k, l \in \mathbb{N}_{0}} \frac{\epsilon^{k+l+1}}{k ! l !(k+l+1)} \varphi^{\langle k\rangle}(0) \psi^{\langle l+2 m\rangle}(0)+ \\
& \quad+\exp \left(-\frac{1}{2} \frac{\epsilon^{2}}{\tau}\right) \mathcal{O}(\tau) .
\end{aligned}
$$


Substituting equations $(\overline{\mathrm{A} .20})$ and $(\mathrm{A.22})$ to equation $(\mathrm{A} .18)$ and ignoring exponentially suppressed terms $\exp \left(-\frac{1}{2} \frac{\epsilon^{2}}{\tau}\right) \mathcal{O}(\tau)$ we obtain the desired relation A.17.

Next we will prove another expansion for small $\tau$

$$
\begin{aligned}
& \frac{1}{\sqrt{2 \pi \nu \tau}} \omega\left(\frac{\xi \zeta}{\xi+\zeta}\right) \exp \left(-\frac{1}{2 \nu \tau}(\xi+\zeta)^{2}\right) \mu(\xi) \mu(\zeta)= \\
& \quad=\sum_{n \in \mathbb{N}_{0}}\left(\frac{\tau \nu}{2}\right)^{\frac{n+1}{2}} \frac{1}{\Gamma\left(\frac{n+1}{2}\right)} \sum_{\substack{m, k, l \in \mathbb{N}_{0} \\
k+l+m=n}} \frac{\omega^{\langle m\rangle}(0)}{n+m+1} \frac{\left(\begin{array}{c}
m+k \\
k
\end{array}\right)\left(\begin{array}{c}
m+l \\
l
\end{array}\right)}{\left(\begin{array}{c}
n+m \\
m
\end{array}\right)} \dddot{\partial}^{\langle k\rangle} \bullet \mathcal{D} \bullet \breve{\partial}^{\langle l\rangle},
\end{aligned}
$$

where $\omega$ is some smooth function. As a corollary for $\omega=1$ we get

$$
\begin{aligned}
& \frac{1}{\sqrt{2 \pi \nu \tau}} \exp \left(-\frac{1}{2 \nu \tau}(\xi+\zeta)^{2}\right) \mu(\xi) \mu(\zeta)=
\end{aligned}
$$

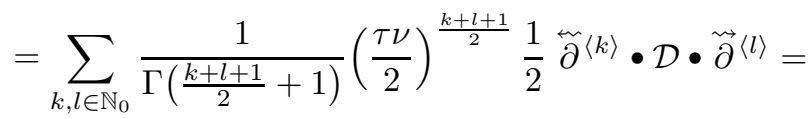

$$
\begin{aligned}
& =\sum_{m \in \mathbb{N}} \frac{1}{m !}\left(\frac{\tau \nu}{2}\right)^{m} \frac{1}{2} \sum_{\substack{k, l \in \mathbb{N}_{0} \\
k+l+1=2 m}} \tilde{\partial}^{\langle k\rangle} \bullet \mathcal{D} \bullet \stackrel{\tilde{\partial}}{ }^{\langle l\rangle}+ \\
& +\sum_{m \in \mathbb{N}_{0}} \frac{1}{\Gamma\left(m+\frac{3}{2}\right)}\left(\frac{\tau \nu}{2}\right)^{m+\frac{1}{2}} \frac{1}{2} \sum_{\substack{k, l \in \mathbb{N}_{0} \\
k+l=2 m}} \stackrel{\ddot{\partial}}{\langle k\rangle}^{\langle k} \mathcal{D} \cdot \stackrel{\check{\partial}}{ }^{\langle l\rangle} .
\end{aligned}
$$

As before, we drop the factor $\nu$ in the proof because it can easily be restored from the $\tau$ dependence. Again, we smooth the relation with test functions $\varphi$ and $\psi$. Thanks to the exponential suppression, only small values of $\xi$ and $\zeta$ contributes to the integrals. Therefore we can rescale $\xi$ and $\zeta$ by $\sqrt{\tau}$, expand $\varphi, \psi$, and $\omega$ about zero, and get

$$
\begin{gathered}
\frac{1}{2 \pi \tau} \int_{\xi, \zeta \in \mathbb{R}^{+}} \varphi(\xi) \psi(\zeta) \omega\left(\frac{\xi \zeta}{\xi+\zeta}\right) \exp \left(-\frac{1}{2 \tau}(\xi+\zeta)^{2}\right) d \xi d \zeta= \\
=\frac{1}{2 \pi \tau} \sum_{n \in \mathbb{N}_{0}} \tau^{\frac{n}{2}+1} \sum_{\substack{k, l, m \in \mathbb{N}_{0} \\
k+l+m=n}} \frac{n !}{k ! l ! m !} \varphi^{\langle k\rangle}(0) \psi^{\langle l\rangle}(0) \omega^{\langle m\rangle}(0) \times \\
\times \frac{1}{n !} \int_{\xi, \zeta \mathbb{R}^{+}} d \xi d \zeta \frac{\xi^{m+k} \zeta^{m+l}}{(\xi+\zeta)^{m}} \exp \left(-\frac{1}{2}(\xi+\zeta)^{2}\right)= \\
=\sum_{n \in \mathbb{N}_{0}}\left(\frac{\tau}{2}\right)^{\frac{n+1}{2}} \frac{1}{\Gamma\left(\frac{n+1}{2}\right)} \sum_{\substack{k, l, m \in \mathbb{N}_{0} \\
k+l+m=n}} \frac{\omega^{\langle m\rangle}(0)}{n+m+1} \frac{\left(\begin{array}{c}
m+k \\
k
\end{array}\right)\left(\begin{array}{c}
m+l \\
l
\end{array}\right)}{\left(\begin{array}{c}
n+m \\
m
\end{array}\right)} \varphi^{\langle k\rangle}(0) \psi^{\langle l\rangle}(0)
\end{gathered}
$$

what proves the relation (A.23). Here we have used the integral (C.18).

\section{Manifold with boundary - no reflection contribution}

Now we find an expansion of the contribution to the short time amplitude from the trajectories near the geodesic without reflection in the domain $\Omega$ with boundary $\partial \Omega$. We will prove for small $\tau$

$$
\begin{aligned}
& \frac{n}{(2 \pi \nu \tau)^{\frac{d}{2}}} \mathfrak{g}^{\frac{1}{2}}(x) \mathfrak{g}^{\frac{1}{2}}(z) \Delta(x \mid z) \exp \left(-\frac{1}{\nu \tau} \sigma(x \mid z)\right)= \\
&=\mathcal{G}+\sqrt{\tau}\left(-\frac{1}{n} \sqrt{\frac{\nu}{2 \pi}}\right) \mathcal{Q}-\tau \frac{\nu}{2} \stackrel{\sim m}{\mathcal{L}}+\mathcal{O}\left(\tau^{\frac{3}{2}}\right) .
\end{aligned}
$$


As usual, we will be proving a smoothed version of this relation - we multiply the expression by test functions $\varphi(x)$ and $\psi(z)$, and integrate over $x$ and $z$. Thanks to the exponential suppression the only non-trivial contribution is from $x \approx z$. Therefore it is sufficient to prove the relation locally. Clearly, for $\varphi$ and $\psi$ with support inside of interior of the domain $\Omega$ the boundary does not have any influence and the relation reduces to the case without boundary. Therefore we will investigate only the case when $\varphi$ and $\psi$ are localized near the boundary. Thanks to locality we can also, without losing generality, assume that the test functions are localized on the neighborhood $U \subset \Omega$ of the boundary with topology $\mathbb{R} \times \partial \Omega$ on which the geodesics normal to the boundary do not cross. In such a neighborhood we can use the method described in appendix $B$ and change the integration over a neighborhood to integration over the boundary $\partial \Omega$ and geodesic distance from the boundary $(\operatorname{see}(\mathrm{B} .60))$

$$
\begin{array}{ll}
x \rightarrow \widehat{x}, \xi \quad, \quad x=\widehat{\boldsymbol{w}}(\widehat{x}, \xi) \\
z \rightarrow \bar{z}, \zeta \quad, \quad y=\widehat{\boldsymbol{w}}(\widehat{z}, \zeta)
\end{array}
$$

The Jacobian associated with this change of variables is

$$
\mathfrak{g}^{\frac{1}{2}}(\widehat{x})=d \xi \mathfrak{q}^{\frac{1}{2}}(\widehat{x}, \xi)=d \xi \hat{j}(\widehat{x}, \xi) \mathfrak{q}^{\frac{1}{2}}
$$

Here we use the convention (B.62) - we denote the spacetime dependent object $A(x)$ in variables $\widehat{x}, \xi$ and tensor indices moved to the boundary as $\hat{A}(\widehat{x}, \xi)$. Changing variables we get

$$
\begin{aligned}
& \frac{n}{(2 \pi \nu \tau)^{\frac{d}{2}}} \int_{x, z \in \Omega} \mathfrak{g}^{\frac{1}{2}}(x) \mathfrak{g}^{\frac{1}{2}}(z) \Delta(x \mid z) \exp \left(-\frac{1}{\nu \tau} \sigma(x \mid z)\right) \varphi(x) \psi(z)= \\
& =\frac{n}{(2 \pi \nu \tau)^{\frac{d}{2}}} \int_{\substack{\widehat{x}, \bar{z} \in \partial \Omega \\
\xi, \zeta \in \mathbb{R}^{+}}} d \xi d \zeta \hat{j}(\widehat{x}, \xi) \mathfrak{q}^{\frac{1}{2}}(\widehat{x}) \hat{j}(\widehat{z}, \zeta) \mathfrak{q}^{\frac{1}{2}}(z) \hat{\Delta}(\widehat{x}, \xi \mid \bar{z}, \zeta) \times \\
& \quad \times \exp \left(-\frac{1}{\nu \tau} \hat{\sigma}(\widehat{x}, \xi \mid \bar{z}, \zeta)\right) \hat{\varphi}(\widehat{x}, \xi) \hat{\psi}(\bar{z}, \zeta)= \\
& =\int_{\widehat{x} \in \partial \Omega} \mathfrak{q}^{\frac{1}{2}(\widehat{x})} \frac{n}{(2 \pi \nu \tau)^{\frac{1}{2}}} \int_{\xi, \zeta \in \mathbb{R}^{+}} d \xi d \zeta \hat{\varphi}(\widehat{x}, \xi) \times \\
& \quad \times \frac{1}{(2 \pi \nu \tau)^{\frac{d}{2}}} \int_{z \in \partial \Omega} \mathfrak{q}^{\frac{1}{2}}(\bar{z}) \Delta(\widehat{x} \mid \bar{z}) \hat{\psi}(\widehat{z}, \zeta) \exp \left(-\frac{1}{\nu \tau} \hat{\sigma}(\widehat{x}, \xi \mid \bar{z}, \zeta)+\hat{l}(\widehat{x}, \xi \mid \bar{z}, \zeta)\right) .
\end{aligned}
$$

Here we have defined

$$
\hat{l}(\widehat{x}, \xi \mid \bar{z}, \zeta)=\ln \left(\hat{j}(\widehat{x}, \xi) \frac{\widehat{\Delta}(\widehat{x}, \xi \mid \bar{z}, \zeta)}{\Delta(\widehat{x} \mid \bar{z})} \hat{j}(\widehat{z}, \zeta)\right),
$$

where $\Delta(\widehat{x} \mid \bar{z})$ is the Van-Vleck Morette determinant of the metric $q$ on the boundary manifold.

Exponential suppression ensures again that the only contributions comes from $\widehat{x} \approx \bar{z}$. So we can change variables $\widehat{x}, \widehat{z} \rightarrow \widehat{y}, Y$

$$
\widehat{y}=\widehat{x} \quad, \quad Y \in \mathbf{T}_{\widehat{y}} \partial \Omega \quad, \quad \widehat{z}=\boldsymbol{v}_{\widehat{y}}(\sqrt{\tau} Y),
$$

with the exponential map $\boldsymbol{v}_{\widehat{y}}(Y)$ defined as in (B.1) but on the boundary manifold. The Jacobian for this change of variables is given by an equation similar to (B.43), only with the Van-Vleck 
Morette determinant $\Delta(\widehat{x}, \widehat{y})$ defined using the metric $q$ on the boundary manifold. We use covariant expansions

$$
\begin{aligned}
\hat{\sigma}(\widehat{y}, \xi \mid \hat{y}, \sqrt{\tau} Y, \zeta) & =\tau n^{2} \frac{1}{2}(\xi-\zeta)^{2}+\sum_{k=2,3, \ldots} \tau^{\frac{k}{2}} \frac{1}{k !} \widehat{\sigma}_{0, k} \boldsymbol{\mu}_{1} \ldots \boldsymbol{\mu}_{k}(\widehat{y} ; \xi, \zeta) Y^{\boldsymbol{\mu}_{1}} \ldots Y^{\boldsymbol{\mu}_{k}} \\
\widehat{l}(\widehat{y}, \xi \mid \widehat{y}, \sqrt{\tau} Y, \zeta) & =\sum_{k=\mathbb{N}_{0}} \tau^{\frac{k}{2}} \frac{1}{k !} \widehat{l}_{0, k} \boldsymbol{\mu}_{1} \ldots \boldsymbol{\mu}_{k}(\widehat{y} ; \xi, \zeta) Y^{\boldsymbol{\mu}_{1}} \ldots Y^{\boldsymbol{\mu}_{k}}, \\
\hat{\psi}(\widehat{y}, \sqrt{\tau} Y, \zeta) & =\sum_{k=\mathbb{N}_{0}} \tau^{\frac{k}{2}} \frac{1}{k !} \widehat{\psi}_{k \boldsymbol{\mu}_{1} \ldots \boldsymbol{\mu}_{k}}(\widehat{y} ; \zeta) Y^{\boldsymbol{\mu}_{1}} \ldots Y^{\boldsymbol{\mu}_{k}}
\end{aligned}
$$

with coefficients given by expressions (B.99 B.103), (B.104 B.106), and (B.27). Expanding the exponential, gathering all terms up to order $\mathcal{O}(\tau)$, and performing a Gaussian integration over $Y$ lead to

$$
\int_{\widehat{y} \in \partial \Omega} \frac{n}{(2 \pi \nu \tau)^{\frac{1}{2}}} \int_{\xi, \zeta \in \mathbb{R}^{+}} d \xi d \zeta \exp \left(-\frac{n^{2}}{2 \nu \tau}(\zeta-\xi)^{2}\right) \tilde{j}(\widehat{y} ; \xi, \zeta)\left(\omega_{0}(\widehat{y} ; \xi, \zeta)+\tau \omega_{1}(\hat{y} ; \xi, \zeta)+\mathcal{O}\left(\tau^{2}\right)\right)
$$

with

$$
\begin{aligned}
& \tilde{j}(\widehat{y} ; \xi \zeta)=\hat{j}(\widehat{y}, \xi) \widehat{\Delta}(\widehat{y}, \xi \mid \widehat{y}, \zeta) \hat{j}(\widehat{y}, \zeta) \mathfrak{q}^{\frac{1}{2}}(\widehat{y})\left(\operatorname{Det}_{\| \prime} \widehat{\sigma}_{0,2}(\widehat{y} ; \xi, \zeta)\right)^{-\frac{1}{2}}= \\
& =(\hat{j}(\widehat{y}, \xi) \widehat{\Delta}(\widehat{y}, \xi \mid \widehat{y}, \zeta) \hat{j}(\widehat{y}, \zeta))^{\frac{1}{2}}, \\
& \omega_{0}(\widehat{y} ; \xi, \zeta)=\widehat{\varphi}(\widehat{y}, \xi) \hat{\psi}(\widehat{y}, \zeta) \\
& \omega_{1}(\widehat{y} ; \xi, \zeta)=\nu \hat{\varphi}(\widehat{y}, \xi) \widehat{\psi}(\widehat{y}, \zeta)\left(-\frac{1}{8} \widehat{\sigma}_{0,4 \mu \nu \kappa \boldsymbol{\lambda}} \widehat{\sigma}_{0,2}^{-1 \mu \nu} \widehat{\sigma}_{0,2}^{-1 \kappa \boldsymbol{\lambda}}+\frac{1}{2} \widehat{l}_{0,2 \mu \nu} \widehat{\sigma}_{0,2}^{-1 \mu \nu}+\right. \\
& +\frac{1}{8} \widehat{\sigma}_{0,3 \boldsymbol{\mu} \boldsymbol{\nu} \boldsymbol{\alpha}} \widehat{\sigma}_{0,3 \boldsymbol{\kappa} \boldsymbol{\lambda} \boldsymbol{\beta}} \widehat{\sigma}_{0,2}^{-1 \boldsymbol{\mu \nu}} \widehat{\sigma}_{0,2}^{-1 \boldsymbol{\alpha} \boldsymbol{\beta}} \widehat{\sigma}_{0,2}^{-1 \kappa \boldsymbol{\lambda}}-\frac{1}{2} \widehat{l}_{0,1 \boldsymbol{\mu}} \widehat{\sigma}_{0,3 \boldsymbol{\kappa} \boldsymbol{\lambda} \boldsymbol{\nu}} \widehat{\sigma}_{0,2}^{-1 \boldsymbol{\mu} \boldsymbol{\nu}} \widehat{\sigma}_{0,2}^{-1 \kappa \boldsymbol{\lambda}}+ \\
& \left.+\frac{1}{12} \widehat{\sigma}_{0,3 \boldsymbol{\mu} \boldsymbol{\alpha} \boldsymbol{\alpha}} \widehat{\sigma}_{0,3 \boldsymbol{\nu} \boldsymbol{\lambda} \boldsymbol{\beta}} \widehat{\sigma}_{0,2}^{-1 \boldsymbol{\mu} \boldsymbol{\nu}} \widehat{\sigma}_{0,2}^{-1 \boldsymbol{\alpha} \boldsymbol{\beta}} \widehat{\sigma}_{0,2}^{-1 \boldsymbol{\kappa} \boldsymbol{\lambda}}+\frac{1}{2} \widehat{l}_{0,1 \boldsymbol{\mu}} \widehat{l}_{0,1 \boldsymbol{\nu}} \widehat{\sigma}_{0,2}^{-1 \mu \nu}\right)(\hat{y} ; \xi, \zeta)+ \\
& +\nu \hat{\varphi}(\widehat{y} ; \xi) \widehat{\psi}_{1 \boldsymbol{\mu}}(\widehat{y} ; \zeta) \widehat{\sigma}_{0,2}^{-1 \mu \nu}\left(\widehat{l}_{0,1 \nu}-\frac{1}{2} \widehat{\sigma}_{0,3 \kappa \lambda \nu} \widehat{\sigma}_{0,2}^{-1 \kappa \lambda}\right)(\widehat{y} ; \xi, \zeta)+ \\
& +\nu \widehat{\varphi}(\widehat{y} ; \xi) \widehat{\psi}_{2 \mu \nu}(\widehat{y} ; \zeta) \widehat{\sigma}_{0,2}^{-1 \mu \nu}(\widehat{y} ; \xi, \zeta) \text {. }
\end{aligned}
$$

The equality in (A.36) is proved in (B.76). Applying the expansion in (A.17) in A.35) and using A.6. we get

$$
\begin{aligned}
& \int_{y \in \Omega} \mathfrak{g}^{\frac{1}{2}}(y) \varphi(y) \psi(y)+ \\
& \quad+\sqrt{\tau}\left(-\frac{1}{n} \sqrt{\frac{\nu}{2 \pi}}\right) \int_{\widehat{y} \in \Omega} \mathfrak{q}^{\frac{1}{2}}(\widehat{y}) \varphi(\widehat{y}) \psi(\widehat{y})+ \\
& \quad+\tau \int_{\substack{\hat{y} \in \partial \Omega \\
\eta \in \mathbb{R}^{+}}} d \eta \mathfrak{q}^{\frac{1}{2}}(\widehat{y}) \hat{j}(\hat{y} ; \eta)\left(\frac{\nu}{4 n^{2}} \tilde{j}^{-1}\left(\tilde{j} \omega_{0}\right)^{1,1 \prime}+\frac{\nu}{4 n^{2}} \tilde{j}^{-1}\left(\tilde{j} \omega_{0}\right)^{\mathrm{r} \mathbf{r} \prime}+\omega_{1}\right)(\hat{y} ; \eta, \eta)+ \\
& +\mathcal{O}\left(\tau^{\frac{3}{2}}\right)
\end{aligned}
$$


where $f^{1 \prime}(\xi, \zeta)$ (or $f^{\text {r' }}(\xi, \zeta)$ ) means derivative of a function $f$ with respect of the left (or right) argument.

We see that we have proved already the desired expansion up to order $\sqrt{\tau}$. Now we proceed to prove it in the order $\tau$. We split the integrand of the last term to pieces and compute each of them. First we note that (see (B.101))

$$
\left[\widehat{\sigma}_{0,2}\right]=\widehat{q} \quad,
$$

where by a coincidence limit of a function $f(\xi, \zeta)$ depending on two real parameters we mean $[f](\eta)=$ $f(\eta, \eta)$. Using (B.107) and (B.108) we get

$$
\begin{aligned}
\frac{1}{n}\left[(\ln \tilde{j})^{r^{\prime}}\right] & =\frac{1}{2} n \widehat{k}, \\
\frac{1}{n^{2}}\left[(\ln \tilde{j})^{r / r^{\prime}}\right] & =\frac{1}{2 n^{2}}(\ln \hat{j})^{r / r^{\prime}}+\frac{1}{2 n^{2}}\left[(\ln \widehat{\Delta})^{r / r^{\prime}}\right]=-\frac{n^{2}}{6} \widehat{\mathrm{K}}^{2}+\frac{1}{3} \widehat{k}^{\prime},
\end{aligned}
$$

and therefore

$$
\frac{1}{n^{2}}\left[\tilde{j}^{-1}\left(\tilde{j} \omega_{0}\right)^{r^{\prime} r^{\prime}}\right]=\frac{1}{n^{2}} \widehat{\varphi} \widehat{\psi}^{\prime \prime}+\widehat{k} \widehat{\varphi} \widehat{\psi}^{\prime}+\left(\frac{1}{3} \widehat{k}^{\prime}-\frac{1}{6} n^{2} \widehat{\mathrm{K}}^{2}+\frac{1}{4} \widehat{k}^{2}\right) \widehat{\varphi} \widehat{\psi}
$$

Here $\hat{k}$ is a trace of extrinsic curvature and $\widehat{\mathrm{K}}^{2}$ is a square of the extrinsic curvature (eq. (B.51)). Next, using (B.27), (B.105), and (B.102), transforming the connection $\nabla$ to $\vec{\nabla}$ (eq. (B.64) and performing an integration by parts gives

$$
\begin{gathered}
\frac{\nu}{2} \int_{\partial \Omega} \mathfrak{q}^{\frac{1}{2}} \hat{j} \hat{\varphi}\left(\hat{q}^{-1 \mu \nu} \widehat{\psi}_{2 \mu \nu}+\widehat{\psi}_{1 \mu} \widehat{q}^{-1 \mu \nu}\left(\left[\widehat{l}_{0,1 \nu}\right]-\frac{1}{2}\left[\widehat{\sigma}_{0,3 \kappa \lambda \nu}\right] \widehat{q}^{-1 \kappa \lambda}\right)\right)= \\
=-\frac{\nu}{2} \int_{\partial \Omega} \mathfrak{q}^{\frac{1}{2}} \mathrm{D}_{\boldsymbol{\mu}} \hat{\varphi} \widehat{q}^{-1 \mu \nu} \mathrm{D}_{\boldsymbol{\nu}} \widehat{\psi} .
\end{gathered}
$$

Substituting for $\left[\widehat{\sigma}_{0, k}\right]$ and $\left[\hat{l}_{0, k}\right]$ in remaining terms of $\left[\omega_{1}\right]$, a straightforward long calculation gives

$$
\begin{aligned}
& -\frac{1}{8}\left[\widehat{\sigma}_{0,4 \mu \nu \kappa \lambda}\right] \widehat{q}^{-1 \mu \nu} \widehat{q}^{-1 \kappa \lambda}+\frac{1}{2}\left[\widehat{l}_{0,2 \mu \nu}\right] \widehat{q}^{-1 \mu \nu}+ \\
& +\frac{1}{8}\left[\widehat{\sigma}_{0,3 \boldsymbol{\mu \nu} \boldsymbol{\alpha}}\right]\left[\widehat{\sigma}_{0,3 \boldsymbol{\kappa} \boldsymbol{\lambda} \boldsymbol{\beta}}\right] \widehat{q}^{-1 \boldsymbol{\mu \nu}} \widehat{q}^{-1 \boldsymbol{\alpha} \boldsymbol{\beta}} \widehat{q}^{-1 \kappa \boldsymbol{\lambda}}+\frac{1}{2}\left[\widehat{l}_{0,1 \boldsymbol{\mu}}\right]\left[\widehat{l}_{0,1 \boldsymbol{\nu}}\right] \widehat{q}^{-1 \boldsymbol{\mu \nu}}+
\end{aligned}
$$

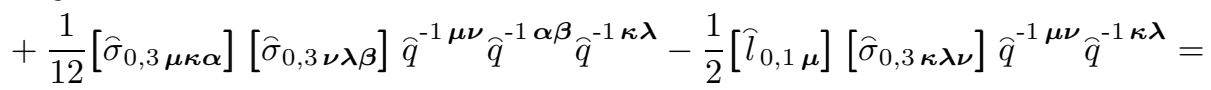

$$
\begin{aligned}
& =\nu\left(-\frac{1}{6} \widehat{k}^{\prime}+\frac{n^{2}}{12} \widehat{\mathrm{K}}^{2}-\frac{n^{2}}{8} \widehat{k}^{2}\right) \text {. }
\end{aligned}
$$

Putting together A.42 A.44, and integrating by parts we find

$$
\begin{aligned}
& \int_{\substack{y \in \partial \Omega \\
\eta \in \mathbb{R}^{+}}} d \eta \mathfrak{q}^{\frac{1}{2}}(\widehat{y}) \hat{j}(\widehat{y} ; \eta)\left(\frac{\nu}{4 n^{2}} \tilde{j}^{-1}\left(\tilde{j} \omega_{0}\right)^{1 / 1 \prime}+\frac{\nu}{4 n^{2}} \tilde{j}^{-1}\left(\tilde{j} \omega_{0}\right)^{\mathrm{r} / \mathrm{r} \prime}+\omega_{1}\right)(\hat{y} ; \eta, \eta)= \\
& =\nu \int_{\mathbb{R}^{+}} d \eta \int_{\partial \Omega} \mathfrak{q}^{\frac{1}{2}}\left(\frac{1}{4 n^{2}}\left(\widehat{\varphi}^{\prime \prime} \hat{\psi}+\widehat{\varphi} \widehat{\psi}^{\prime \prime}+n^{2} \widehat{k}\left(\widehat{\varphi}^{\prime} \hat{\psi}+\widehat{\varphi} \widehat{\psi}^{\prime}\right)\right)-\frac{1}{2} \mathrm{D}_{\boldsymbol{\mu}} \widehat{\varphi} \widehat{q}^{-1 \mu \nu} \mathrm{D}_{\boldsymbol{\nu}} \widehat{\psi}\right)= \\
& =-\frac{\nu}{2} \int_{\mathbb{R}^{+}} d \eta \int_{\partial \Omega} n \widehat{\mathfrak{q}}^{\frac{1}{2}}\left(\frac{1}{n^{2}} \widehat{\varphi}^{\prime} \widehat{\psi}^{\prime}+\mathrm{D}_{\boldsymbol{\mu}} \hat{\varphi} \widehat{q}^{-1 \mu \nu} \mathrm{D}_{\boldsymbol{\nu}} \widehat{\psi}\right)-\frac{\nu}{4 n^{2}} \int_{\partial \Omega} \mathfrak{q}^{\frac{1}{2}}\left(\widehat{\varphi}^{\prime} \hat{\psi}+\hat{\varphi} \widehat{\psi}^{\prime}\right)=
\end{aligned}
$$




$$
\begin{aligned}
& =-\frac{\nu}{2} \int_{\Omega} \mathfrak{g}^{\frac{1}{2}} \mathrm{~d}_{\boldsymbol{\mu}} \varphi g^{-1 \mu \nu} \mathrm{d}_{\boldsymbol{\nu}} \psi-\frac{\nu}{4 n^{2}} \int_{\partial \Omega} \mathfrak{q}^{\frac{1}{2}}\left(\psi \vec{n}^{\mu} \mathrm{d}_{\boldsymbol{\mu}} \varphi+\varphi \vec{n}^{\mu} \mathrm{d}_{\boldsymbol{\mu}} \psi\right)=
\end{aligned}
$$

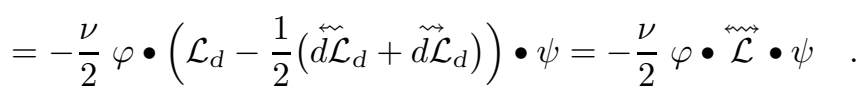

This concludes the proof of the expansion in (A.26).

\section{Manifold with boundary — reflection contribution}

Finally we will prove the last expansion we have needed in the main text:

$$
\begin{aligned}
& \frac{n}{(2 \pi \nu \tau)^{\frac{d}{2}}} \Delta_{\mathrm{b}}^{1-p}(x \mid z) \beta(\tau, x \mid z) \exp \left(-\frac{1}{\nu \tau} \sigma_{\mathrm{b}}(x \mid z)\right)= \\
& \quad=\sqrt{\tau} \frac{1}{n} \sqrt{\frac{\nu}{2 \pi}} \mathcal{Q}-\tau \frac{\nu}{2} \frac{1}{2}\left(\tilde{d \mathcal{F}}_{d}+\widetilde{d \mathcal{F}}_{d}\right)-\tau \frac{\nu}{2}\left(\frac{1+p}{3} k+\beta \text {-terms }\right)+\mathcal{O}\left(\tau^{\frac{3}{2}}\right) .
\end{aligned}
$$

Similarly to the previous section, we smooth this expression with test functions, perform a change of variables (A.27) and consequently (A.31), and use the covariant expansions (A.34),

$$
\begin{aligned}
\widehat{\beta}(\tau, \widehat{x}, \xi \mid \bar{z}, \zeta) & =\widehat{\beta}_{0}(\widehat{x}, \xi \mid \widehat{z}, \zeta)+\sqrt{\tau} \widehat{\beta}_{\frac{1}{2}}(\widehat{x}, \xi \mid \bar{z}, \zeta)+\mathcal{O}(\tau) \\
\hat{\beta}_{0}(\widehat{y}, \xi \mid \widehat{y}, \sqrt{\tau} Y, \zeta) & =\sum_{k=\mathbb{N}_{0}} \tau^{\frac{k}{2}} \frac{1}{k !} \widehat{\beta}_{0 ; 0, k} \boldsymbol{\mu}_{1} \ldots \boldsymbol{\mu}_{k}(\widehat{y} ; \xi, \zeta) Y^{\boldsymbol{\mu}_{1}} \ldots Y^{\boldsymbol{\mu}_{k}}
\end{aligned}
$$

similarly for $\beta_{\frac{1}{2}}(x \mid z)$; and

$$
\begin{aligned}
& \widehat{\sigma}_{\mathrm{b}}(\widehat{y}, \xi \mid \widehat{y}, \sqrt{\tau} Y, \zeta)=\tau n^{2} \frac{1}{2}(\xi+\zeta)^{2}+\sum_{k=2,3, \ldots} \tau^{\frac{k}{2}} \frac{1}{k !} \widehat{\sigma}_{\mathrm{b} 0, k \boldsymbol{\mu}_{1} \ldots \boldsymbol{\mu}_{k}}(\widehat{y} ; \xi, \zeta) Y^{\boldsymbol{\mu}_{1}} \ldots Y^{\boldsymbol{\mu}_{k}} \\
& \widehat{l}_{\mathrm{b}}(\widehat{y}, \xi \mid \widehat{y}, \sqrt{\tau} Y, \zeta)=\sum_{k=\mathbb{N}_{0}} \tau^{\frac{k}{2}} \frac{1}{k !} \widehat{l}_{\mathrm{b} 0, k \boldsymbol{\mu}_{1} \ldots \boldsymbol{\mu}_{k}}(\widehat{y} ; \xi, \zeta) Y^{\boldsymbol{\mu}_{1}} \ldots Y^{\boldsymbol{\mu}_{k}}
\end{aligned}
$$

where

$$
\widehat{l}_{\mathrm{b}}(\widehat{x}, \xi \mid \widehat{z}, \zeta)=\ln \left(\hat{j}(\widehat{x}, \xi) \frac{\widehat{\Delta}_{\mathrm{b}}^{1-p}(\widehat{x}, \xi \mid \bar{z}, \zeta)}{\Delta(\widehat{x} \mid \bar{z})} \hat{j}(\bar{z}, \zeta)\right) .
$$

This leads to a Gaussian integration in the variable $Y \in \mathbf{T}_{\widehat{y}} \partial \Omega$ in which only leading terms in expansions survive and we get

$$
\begin{aligned}
& \frac{n}{(2 \pi \nu \tau)^{\frac{d}{2}}} \int_{x, z \in \Omega} \mathfrak{g}^{\frac{1}{2}}(x) \mathfrak{g}^{\frac{1}{2}}(z) \varphi(x) \psi(z) \Delta_{\mathrm{b}}^{1-p}(x \mid z) \beta(\tau, x \mid z) \exp \left(-\frac{1}{\nu \tau} \sigma_{\mathrm{b}}(x \mid z)\right)= \\
& =\int_{\widehat{y} \in \partial \Omega} \frac{n}{(2 \pi \nu \tau)^{\frac{1}{2}}} \mathfrak{q}^{\frac{1}{2}}(\widehat{y}) \int_{\xi, \zeta \in \mathbb{R}^{+}} d \xi d \zeta \exp \left(-\frac{n^{2}}{2 \nu \tau}(\xi+\zeta)^{2}\right) \hat{\varphi}(\widehat{y}, \xi) \hat{\psi}(\widehat{y}, \zeta) \times \\
& \times \tilde{j}_{\mathrm{b}}\left(\hat{y} ; \xi, \frac{\xi \zeta}{\xi+\zeta}, \zeta\right)\left(\tilde{\beta}_{0}\left(\hat{y} ; \xi, \frac{\xi \zeta}{\xi+\zeta}, \zeta\right)+\sqrt{\tau} \tilde{\beta}_{\frac{1}{2}}\left(\hat{y} ; \xi, \frac{\xi \zeta}{\xi+\zeta}, \zeta\right)+\mathcal{O}(\tau)\right)
\end{aligned}
$$

where

$$
\begin{aligned}
\tilde{j}_{\mathrm{b}}\left(\widehat{y} ; \xi, \frac{\xi \zeta}{\xi+\zeta}, \zeta\right) & =\widehat{j}(\widehat{y}, \xi) \widehat{\Delta}_{\mathrm{b}}^{1-p}(\widehat{y} ; \xi \mid \widehat{y}, \zeta) \widehat{j}(\widehat{y}, \zeta) \mathfrak{q}^{\frac{1}{2}}(\widehat{y})\left(\operatorname{Det}_{\| 1} \widehat{\sigma}_{\mathrm{b} 0,2}(\widehat{y} ; \xi, \zeta)\right)^{-\frac{1}{2}}= \\
& =\left(\widehat{j}(\widehat{y}, \xi) \widehat{\Delta}_{\mathrm{b}}^{1-2 p}(\widehat{y} ; \xi \mid \widehat{y}, \zeta) \widehat{j}(\widehat{y}, \zeta)\right)^{\frac{1}{2}}, \\
\tilde{\beta}_{0}\left(\widehat{y} ; \xi, \frac{\xi \zeta}{\xi+\zeta}, \zeta\right) & =\widehat{\beta}_{0}(\widehat{y}, \xi \mid \widehat{y}, \zeta)
\end{aligned}
$$


with $\tilde{j}_{\mathrm{b}}$ and $\tilde{\beta}_{0}$ depending analyticaly on their three real arguments. $\tilde{\beta}_{\frac{1}{2}}$ is defined in similar way as $\tilde{\beta}_{0}$. Here we anticipate that $\widehat{\Delta}_{\mathrm{b}}$ and $\widehat{\beta}$ can have more complicated analytical dependence on $\xi$ and $\zeta$. The equality in (A.52) follows from (B.98). Using the expansion A.23 we obtain

$$
\begin{aligned}
\int_{\widehat{y} \in \partial \Omega} \mathfrak{q}^{\frac{1}{2}}(\widehat{y})( & \sqrt{\tau} \frac{1}{n} \sqrt{\frac{\nu}{2 \pi}} \tilde{\beta}_{0}(\widehat{y} ; 0,0,0) \varphi(\widehat{y}) \psi(\widehat{y})+ \\
& +\tau \frac{\nu}{4 n^{2}} \tilde{\beta}_{0}(\widehat{y} ; 0,0,0)\left(\hat{\varphi}^{\prime}(\widehat{y}, 0) \hat{\psi}(\widehat{y}, 0)+\hat{\varphi}(\widehat{y}, 0) \hat{\psi}^{\prime}(\widehat{y}, 0)\right)+ \\
& +\tau\left(\frac{\nu}{4 n^{2}}\left(\tilde{j}_{\mathrm{b}} \tilde{\beta}_{0}\right)^{1 \prime}+\frac{\nu}{4 n^{2}}\left(\tilde{j}_{\mathrm{b}} \tilde{\beta}_{0}\right)^{\mathrm{r} \prime}+\right. \\
& \left.+\frac{\nu}{12 n^{2}}\left(\tilde{j}_{\mathrm{b}} \tilde{\beta}_{0}\right)^{\mathrm{m} \prime}+\frac{1}{n} \sqrt{\frac{\nu}{2 \pi}} \tilde{\beta}_{\frac{1}{2}}\right)(\widehat{y} ; 0,0,0) \varphi(\widehat{y}) \psi(\widehat{y})+ \\
& \left.+\mathcal{O}\left(\tau^{\frac{3}{2}}\right)\right)
\end{aligned}
$$

Using the expansion (B.116) of $\tilde{j}_{\mathrm{b}}$ and obvious relations for $\tilde{\beta}_{0}(\hat{y} ; 0,0,0)$ and $\tilde{\beta}_{\frac{1}{2}}(\hat{y} ; 0,0,0)$, this expression is equal to

$$
\begin{aligned}
\int_{\widehat{y} \in \partial \Omega} \mathfrak{q}^{\frac{1}{2}}(\widehat{y})( & \sqrt{\tau} \frac{1}{n} \sqrt{\frac{\nu}{2 \pi}} \beta(0, \widehat{y} \mid \widehat{y}) \varphi(\widehat{y}) \psi(\widehat{y})+ \\
& +\tau \frac{\nu}{4 n^{2}} \beta(0, \widehat{y} \mid \widehat{y})\left(\widehat{\varphi}^{\prime}(\widehat{y}, 0) \widehat{\psi}(\widehat{y}, 0)+\widehat{\varphi}(\widehat{y}, 0) \widehat{\psi}^{\prime}(\widehat{y}, 0)\right)+ \\
& +\frac{\tau \nu}{2}\left(-\frac{1+6}{3} \beta(0, \widehat{y} \mid \widehat{y}) k(\widehat{y})+\frac{2}{n} \sqrt{\frac{1}{2 \pi \nu}} \dot{\beta}(0, \widehat{y} \mid \widehat{y})+\right. \\
& \left.+\left(\frac{1}{2 n^{2}} \tilde{\beta}_{0}{ }^{1 \prime}+\frac{1}{2 n^{2}} \tilde{\beta}_{0}{ }^{\prime \prime}+\frac{1}{6 n^{2}} \tilde{\beta}_{0}{ }^{\mathrm{m} \prime}\right)(\widehat{y} ; 0,0,0)\right) \varphi(\widehat{y}) \psi(\widehat{y})+ \\
& \left.+\mathcal{O}\left(\tau^{\frac{3}{2}}\right)\right)
\end{aligned}
$$

Using the normalization condition 2.15) concludes the proof of the expansion (A.46). By inspection we see that the $\beta$-terms have form

$$
\beta \text {-terms }=-\frac{2}{n} \sqrt{\frac{1}{2 \pi \nu}} \dot{\beta}(0, \widehat{y} \mid \widehat{y})-\left(\frac{1}{2 n^{2}} \tilde{\beta}_{0}{ }^{1 \prime}+\frac{1}{2 n^{2}} \tilde{\beta}_{0}{ }^{\mathrm{r} \prime}+\frac{1}{6 n^{2}} \tilde{\beta}_{0}{ }^{\mathrm{m} \prime}\right)(\widehat{y} ; 0,0,0)
$$




\section{B Geodesic theory}

\section{Basic definitions}

In this appendix we review some facts from geodesic theory and list a number of useful expansion, some of which we have used in this work. The material related to a manifold without boundary is well known - see for example the classical works [15, 16] and [17]. The theory of expansion near a boundary is less known. Some material can be found in 18 20]. The calculations are usually straightforward but cumbersome. We will present mostly only results. But all expressions presented were computed and checked by the author (see [21]).

We start with introducing the covariant expansion in a curved manifold. We would like to expand a sufficiently smooth tensor field $A_{\boldsymbol{\beta} \ldots}^{\boldsymbol{\alpha} \ldots}$ on a manifold around a point $x$. First we change the dependence on a point $z$ in the manifold $M$ to the dependence on a vector $Z$ from $\mathbf{T}_{x} M$, then we transform vector indices from different tangent spaces to one common tensor space, and finally we do the usual Taylor expansion of a linear-space-valued function on a vector space.

To transform the tensor field on the manifold to a linear-space-valued function we need to know how to move tensors from one tangent point to another. We assume that we have given a metric $g$ which define a parallel transport. It allows us to transform tensors from the tangent space at point $z$ in a normal neighborhood of the point $x$ to the space $\mathbf{T}_{x} M$ along the geodetic joining these two points. In the normal neighborhood of $x$ we can parametrize a geodesic by its tangent vector at $x$, i.e. we can define an exponential map $\boldsymbol{u}_{x}$

$$
\begin{gathered}
\boldsymbol{u}_{x}: \mathbf{T}_{x} M \rightarrow M \\
\frac{\nabla}{d \tau} \frac{\mathrm{D}}{d \tau} \boldsymbol{u}_{x}(\tau X)=0 \quad,\left.\quad \frac{\mathrm{D}}{d \tau} \boldsymbol{u}_{x}(\tau X)\right|_{\tau=0}=X
\end{gathered}
$$

If $f(z)$ is some manifold dependent function, we use notation $f(x ; Z)=f\left(\boldsymbol{u}_{x}(Z)\right)$. This transformation concludes our first step. Next we parallel transform vector indices of the tensor field to the space $\mathbf{T}_{x} M$ along the geodesics starting from $x$. We define the tensor of geodesic transport $\iota(x \mid z)$ from $z$ to $x$

$$
\iota^{\boldsymbol{\mu}}{ }_{\nu}(x \mid z) \in \mathbf{T}_{x} M \otimes \mathbf{T}_{z}^{\star} M \quad, \quad \frac{\nabla}{d \tau} \iota\left(x, \boldsymbol{u}_{x}(\tau X)\right)=0 \quad,
$$

and its version with indices up and down

$$
\bar{\iota}=\iota \cdot g^{-1} \quad, \quad \underline{\iota}=g \cdot \iota \quad .
$$

Using this tensor we can write down the tensor field $A$ with transported indices explicitly. We obtain the linear-space-valued function on a linear space

$$
A_{\nu \ldots}^{\boldsymbol{\mu} \ldots}(x ; .) \iota^{\boldsymbol{\alpha}}{ }_{\boldsymbol{\mu}}(x \mid x ; .) \ldots \iota^{-1 \boldsymbol{\nu}}{ }_{\boldsymbol{\beta}}(x \mid x ; .) \ldots \quad: \mathbf{T}_{x} M \rightarrow \mathbf{T}_{x l}^{k} M .
$$

Finally we can write the covariant expansion

$$
A_{\boldsymbol{\nu} \ldots}^{\boldsymbol{\mu} \ldots}(x ; Z) \iota^{\boldsymbol{\alpha}}{ }_{\boldsymbol{\mu}}(x \mid x ; Z) \ldots \iota^{-1 \boldsymbol{\nu}}{ }_{\boldsymbol{\beta}}(x \mid x ; Z) \ldots=\sum_{k \in \mathbb{N}_{0}} \frac{1}{k !} A_{k} \underset{\boldsymbol{\beta} \ldots \boldsymbol{\mu}_{1} \ldots \boldsymbol{\mu}_{k}}{\boldsymbol{\alpha} \ldots}(x) Z^{\boldsymbol{\mu}_{1}} \ldots Z^{\boldsymbol{\mu}_{k}} .
$$


We call $A_{k}(x)$ the coefficients of the covariant expansion of the field $A$ at $x$. They are tensors at $x$ symmetric in indices $\boldsymbol{\mu}_{1}, \ldots, \boldsymbol{\mu}_{k}$.

To compute these coefficients we need to develop geodesic theory to greater detail. First we define the world function $\sigma(x \mid z)$ of the metric $g$. It is given by half of the squared geodesic distance between points $x$ and $z$ - see (1.18). For time-like separated points it is negative. The geodesic distance is then given by

$$
s(x \mid z)=|2 \sigma(x \mid z)|^{\frac{1}{2}} \quad .
$$

We define geodesic tangent vectors $\vec{\sigma}, \overleftarrow{\sigma}$

$$
\vec{\sigma}(x \mid z)=g^{-1}(x) \cdot \mathrm{d}_{1} \sigma(x \mid z) \quad, \quad \overleftarrow{\sigma}(x \mid z)=\mathrm{d}_{\mathrm{r}} \sigma(x \mid z) \cdot g^{-1}(z)
$$

Here, as before, $\mathrm{d}_{\mathrm{l}} f$ or $\mathrm{d}_{\mathrm{r}} f$ denote the gradient in the left or right argument of a bi-function $f(x \mid z)$.

The basic properties of the world function (see e.g. [16]) are that its gradient vector $\vec{\sigma}(x \mid z)$ is really tangent to the geodesic between $x$ and $z$ and it is normalized to the length of the geodesic. I.e.

$$
-Z=\vec{\sigma}(x \mid x ; Z)
$$

We also introduce a special notation for the second derivatives of the world function

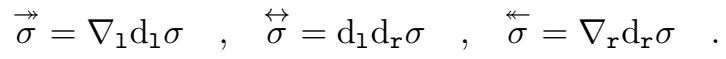

To conclude our definition we introduce also determinants of $\iota, \underline{\iota}, \stackrel{\leftrightarrow}{\sigma}$. They are well-defined objects — bi-densities on $M$

$$
\begin{gathered}
\mathfrak{i}(x \mid z)=\frac{1}{n^{2}} \operatorname{Det} \iota(x \mid z)=\mathfrak{g}^{-\frac{1}{2}}(x) \mathfrak{g}^{\frac{1}{2}}(z) \quad, \quad \underline{\mathfrak{i}}(x \mid z)=\frac{1}{n^{2}} \operatorname{Det} \underline{\iota}(x \mid z)=\mathfrak{g}^{\frac{1}{2}}(x) \mathfrak{g}^{\frac{1}{2}}(z), \\
\mathfrak{s}(x \mid z)=\frac{1}{n^{2}} \operatorname{Det}(-\stackrel{\leftrightarrow}{\sigma}(x \mid z)) .
\end{gathered}
$$

Finally we define Van-Vleck Morette determinant

$$
\Delta(x \mid z)=\mathfrak{s}(x \mid z) \underline{\mathfrak{i}}^{-1}(x \mid z)=\mathfrak{s}(x \mid z) \mathfrak{g}^{-\frac{1}{2}}(x) \mathfrak{g}^{-\frac{1}{2}}(z) .
$$

For a bi-tensor $F(x \mid z)$ on the manifold - a tensor object depending on two points in the manifold - we denote the coincidence limit

$$
[F](x)=F(x \mid x) \quad .
$$

The generalized Synge's theorem (see e.g. [22]) tells us that

$$
\nabla[F]=\left[\nabla_{1} F\right]+\left[\nabla_{\mathrm{r}} F\right] .
$$

\section{Coincidence limits and covariant expansions}

Equation (B.9) gives]

$$
\sigma=\frac{1}{2} \mathrm{~d}_{\mathrm{r}} \sigma \cdot g^{-1} \cdot \mathrm{d}_{\mathrm{r}} \sigma=\frac{1}{2} \mathrm{~d}_{\mathrm{l}} \sigma \cdot g^{-1} \cdot \mathrm{d}_{\mathrm{l}} \sigma=\frac{1}{2} \vec{\sigma} \cdot g \cdot \vec{\sigma}=\frac{1}{2} \overleftarrow{\sigma} \cdot g \cdot \overleftarrow{\sigma}
$$

Taking repeatedly derivatives of this expression in both arguments we can derive

$$
\vec{\sigma} \cdot g=\vec{\sigma} \cdot \vec{\sigma}=\overleftrightarrow{\sigma} \cdot \overleftarrow{\sigma} \quad, \quad g \cdot \overleftarrow{\sigma}=\overleftrightarrow{\sigma} \cdot \overleftarrow{\sigma}=\vec{\sigma} \cdot \overleftrightarrow{\sigma}
$$


and following identities

$$
\begin{aligned}
& \vec{\sigma}^{\mu} \mathrm{d}_{1} \boldsymbol{\mu} \sigma=2 \sigma, \\
& \vec{\sigma}^{\mu} \nabla_{1 \mu} \vec{\sigma}^{\alpha}=\vec{\sigma}^{\alpha} \\
& \vec{\sigma}^{\mu} \nabla_{1 \mu} \overleftarrow{\sigma}^{\alpha}=\overleftarrow{\sigma}^{\alpha} \\
& \vec{\sigma}^{\mu} \nabla_{1 \boldsymbol{\mu}} \vec{\sigma}_{\boldsymbol{\alpha} \boldsymbol{\beta}}=\vec{\sigma}_{\boldsymbol{\alpha} \boldsymbol{\beta}}-\vec{\sigma}_{\boldsymbol{\alpha} \boldsymbol{\mu}} \vec{\sigma}_{\boldsymbol{\beta} \boldsymbol{\nu}} g^{-1 \mu \nu}-\mathrm{R}_{\boldsymbol{\alpha} \boldsymbol{\mu} \boldsymbol{\beta} \boldsymbol{\nu}} \vec{\sigma}^{\boldsymbol{\mu}} \vec{\sigma}^{\nu},
\end{aligned}
$$

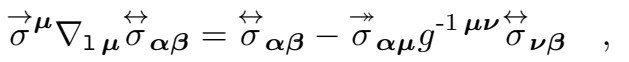

$$
\begin{aligned}
& \vec{\sigma}^{\mu} \nabla_{1} \boldsymbol{\mu} \overleftrightarrow{\sigma}_{\boldsymbol{\alpha} \boldsymbol{\beta}}=\stackrel{\leftarrow}{\sigma}_{\boldsymbol{\alpha} \boldsymbol{\beta}}-g^{-1} \boldsymbol{\mu \nu} \stackrel{\leftrightarrow}{\sigma}_{\boldsymbol{\mu} \boldsymbol{\alpha}} \stackrel{\leftrightarrow}{\sigma}_{\boldsymbol{\nu} \boldsymbol{\beta}} \\
& \vec{\sigma}^{\mu} \nabla_{1 \boldsymbol{\alpha}} \nabla_{1 \boldsymbol{\beta}} \nabla_{1 \boldsymbol{\mu}} \sigma=\vec{\sigma}_{\boldsymbol{\alpha} \boldsymbol{\beta}}-\vec{\sigma}_{\boldsymbol{\alpha} \boldsymbol{\mu}} \vec{\sigma}_{\boldsymbol{\beta} \boldsymbol{\nu}} g^{-1 \mu \nu} \text {. }
\end{aligned}
$$

Similar, more complicated relations hold for higher derivatives. Using the fact that coincidence limits of the world function and tangent geodesic vector are zero, taking coincidence limits of relations above and similar relations for higher derivatives, and using the Synge's theorem, we get

$$
\begin{aligned}
& {[\sigma]=0,} \\
& {\left[\mathrm{~d}_{\mathrm{l}} \sigma\right]=\left[\mathrm{d}_{\mathrm{r}} \sigma\right]=0} \\
& {\left[\nabla_{1} \nabla_{1} \sigma\right]=-\left[\nabla_{1} \nabla_{\mathrm{r}} \sigma\right]=\left[\nabla_{\mathrm{r}} \nabla_{\mathrm{r}} \sigma\right]=g \quad,} \\
& {\left[\nabla_{1} \nabla_{1} \nabla_{1} \sigma\right]=\left[\nabla_{1} \nabla_{1} \nabla_{\mathrm{r}} \sigma\right]=\left[\nabla_{1} \nabla_{\mathrm{r}} \nabla_{\mathrm{r}} \sigma\right]=\left[\nabla_{\mathrm{r}} \nabla_{\mathrm{r}} \nabla_{\mathrm{r}} \sigma\right]=0} \\
& {\left[\nabla_{1 \boldsymbol{\alpha}} \nabla_{1 \boldsymbol{\beta}} \nabla_{1 \boldsymbol{\mu}} \nabla_{1 \boldsymbol{\nu}} \sigma\right]=-\left[\nabla_{1 \boldsymbol{\beta}} \nabla_{1 \boldsymbol{\mu}} \nabla_{1 \boldsymbol{\nu}} \nabla_{\mathrm{r} \boldsymbol{\alpha}} \sigma\right]=\left[\nabla_{1 \boldsymbol{\mu}} \nabla_{1 \boldsymbol{\nu}} \nabla_{\mathrm{r} \boldsymbol{\beta}} \nabla_{\mathrm{r} \boldsymbol{\alpha}} \sigma\right]=} \\
& =-\left[\nabla_{\mathrm{I} \nu} \nabla_{\mathrm{r} \boldsymbol{\mu}} \nabla_{\mathrm{r} \boldsymbol{\beta}} \nabla_{\mathrm{r} \boldsymbol{\alpha}} \sigma\right]=\left[\nabla_{\mathrm{r} \boldsymbol{\nu}} \nabla_{\mathrm{r} \boldsymbol{\mu}} \nabla_{\mathrm{r} \boldsymbol{\beta}} \nabla_{\mathrm{r} \boldsymbol{\alpha}} \sigma\right]=-\frac{1}{3}\left(\mathrm{R}_{\boldsymbol{\alpha} \boldsymbol{\mu} \beta \boldsymbol{\nu}}+\mathrm{R}_{\boldsymbol{\alpha} \boldsymbol{\nu} \boldsymbol{\beta} \boldsymbol{\mu}}\right) .
\end{aligned}
$$

Similar relation for the fifth and sixth derivative can be found in [21].

Now we are prepared to compute at least some coefficients of covariant expansion. We start with the simplest case of the covariant expansion of a function $f$ on the manifold. In this case we do not have problems with tensor nature of $f$ and we do not have to worry about parallel transport of tensor indices. The equation (B.6) can be rewritten using (B.9) as

$$
f(z)=\sum_{k \in \mathbb{N}_{0}} \frac{(-1)^{k}}{k !} f_{k} \boldsymbol{\mu}_{1} \ldots \boldsymbol{\mu}_{k}(x) \vec{\sigma}^{\boldsymbol{\mu}_{1}}(x, z) \ldots \vec{\sigma}^{\boldsymbol{\mu}_{k}}(x, z) .
$$

Taking derivatives of this equation and coincidence limits we can find that the coefficients are given by

$$
f_{k \mu_{1} \ldots \mu_{k}}=\nabla_{\left(\mu_{1} \ldots \nabla_{\left.\mu_{k}\right)} f\right.} .
$$

To do a similar calculation for a general tensor field $A$ we need to know the coincidence limits of the geodesic transport tensor. They can be calculated from the equation

$$
\vec{\sigma}=-\iota \cdot \overleftarrow{\sigma}
$$

by taking derivatives and coincidence limits. We give only a list of some of them (see [17,22] or [21]).

$$
\begin{aligned}
& {[\underline{l}]=g \text {, }} \\
& {\left[\nabla_{1} \underline{\underline{L}}\right]=\left[\nabla_{\mathrm{r} \underline{\imath}}\right]=0} \\
& -\left[\nabla_{1 \boldsymbol{\beta}} \nabla_{1 \boldsymbol{\alpha} \underline{\iota}_{\mu \nu}}\right]=\left[\nabla_{1 \boldsymbol{\alpha}} \nabla_{\mathrm{r} \boldsymbol{\beta} \underline{\iota}_{\mu \nu}}\right]=-\left[\nabla_{\mathrm{r} \boldsymbol{\alpha}} \nabla_{\mathrm{r} \boldsymbol{\beta} \underline{\iota}_{\mu \nu}}\right]=\frac{1}{2} \mathrm{R}_{\boldsymbol{\alpha} \boldsymbol{\beta} \mu \nu}, \\
& {\left[\nabla_{1 \gamma} \nabla_{1 \beta} \nabla_{1 \alpha \underline{\mu}}\right]=-\frac{1}{3} \nabla_{\gamma} R_{\alpha \beta \mu \nu}-\frac{1}{3} \nabla_{\beta} R_{\alpha \gamma \mu \nu},}
\end{aligned}
$$




$$
\begin{aligned}
& {\left[\nabla_{1 \delta} \nabla_{1 \gamma} \nabla_{1 \beta} \nabla_{1 \alpha \underline{\iota}}\right]=} \\
& -\frac{1}{4} \nabla_{\delta} \nabla_{\gamma} \mathrm{R}_{\mu \nu \alpha \beta}-\frac{1}{4} \nabla_{\delta} \nabla_{\beta} \mathrm{R}_{\mu \nu \alpha \gamma}-\frac{1}{4} \nabla_{\gamma} \nabla_{\beta} \mathrm{R}_{\mu \nu \alpha \delta}+ \\
& +\frac{1}{8} \mathrm{R}_{\mu \nu \delta \lambda} \mathrm{R}_{\kappa \boldsymbol{\alpha} \beta \gamma} g^{-1 \kappa \lambda}+\frac{1}{8} \mathrm{R}_{\mu \nu \gamma \lambda} \mathrm{R}_{\kappa \alpha \beta \delta} g^{-1 \kappa \lambda}+\frac{1}{8} \mathrm{R}_{\mu \nu \beta \lambda} \mathrm{R}_{\kappa \alpha \gamma \delta} g^{-1 \kappa \lambda}+ \\
& +\frac{1}{24} \mathrm{R}_{\mu \nu \delta \boldsymbol{\lambda}} \mathrm{R}_{\kappa \beta \alpha \gamma} g^{-1 \kappa \boldsymbol{\lambda}}+\frac{1}{24} \mathrm{R}_{\mu \nu \gamma \lambda} \mathrm{R}_{\kappa \beta \boldsymbol{\alpha} \delta} g^{-1 \kappa \boldsymbol{\lambda}}+\frac{1}{24} \mathrm{R}_{\mu \nu \delta \boldsymbol{\lambda}} \mathrm{R}_{\kappa \gamma \boldsymbol{\alpha} \boldsymbol{\beta}} g^{-1 \kappa \boldsymbol{\lambda}}+ \\
& +\frac{1}{8} \mathrm{R}_{\nu \lambda \gamma \delta} \mathrm{R}_{\kappa \mu \alpha \beta} g^{-1 \kappa \lambda}+\frac{1}{8} \mathrm{R}_{\nu \lambda \beta \delta} \mathrm{R}_{\kappa \mu \alpha \gamma} g^{-1 \kappa \lambda}+\frac{1}{8} \mathrm{R}_{\nu \lambda \beta \gamma} \mathrm{R}_{\kappa \mu \alpha \delta} g^{-1 \kappa \lambda}+ \\
& +\frac{1}{8} \mathrm{R}_{\nu \lambda \alpha \delta} \mathrm{R}_{\kappa \mu \beta \gamma} g^{-1 \kappa \lambda}+\frac{1}{8} \mathrm{R}_{\nu \lambda \alpha \gamma} \mathrm{R}_{\kappa \mu \beta \delta} g^{-1 \kappa \lambda}+\frac{1}{8} \mathrm{R}_{\nu \lambda \alpha \beta} \mathrm{R}_{\kappa \mu \gamma \delta} g^{-1 \kappa \lambda}+ \\
& +\frac{1}{24} \mathrm{R}_{\mu \nu \kappa \gamma} \mathrm{R}_{\boldsymbol{\alpha} \beta \delta \boldsymbol{\lambda}} g^{-1 \kappa \boldsymbol{\lambda}}+\frac{1}{24} \mathrm{R}_{\mu \nu \kappa \beta} \mathrm{R}_{\boldsymbol{\alpha} \gamma \boldsymbol{\delta} \boldsymbol{\lambda}} g^{-1 \kappa \boldsymbol{\lambda}}+\frac{1}{24} \mathrm{R}_{\mu \nu \kappa \beta} \mathrm{R}_{\boldsymbol{\alpha} \delta \boldsymbol{\gamma} \boldsymbol{\lambda}} g^{-1 \kappa \boldsymbol{\lambda}}+ \\
& +\frac{1}{24} \mathrm{R}_{\mu \nu \kappa \alpha} \mathrm{R}_{\boldsymbol{\beta} \gamma \delta \boldsymbol{\lambda}} g^{-1 \kappa \boldsymbol{\lambda}}+\frac{1}{24} \mathrm{R}_{\mu \nu \kappa \alpha} \mathrm{R}_{\boldsymbol{\beta} \delta \gamma \boldsymbol{\lambda}} g^{-1 \kappa \boldsymbol{\lambda}}+\frac{1}{8} \mathrm{R}_{\nu \mu \kappa \boldsymbol{\alpha}} \mathrm{R}_{\boldsymbol{\beta} \boldsymbol{\gamma} \boldsymbol{\delta}} g^{-1 \kappa \boldsymbol{\lambda}}
\end{aligned}
$$

Derivatives in other argument can be obtained using Synge's theorem and commuting covariant derivatives.

Now it is straightforward to compute the coefficients in a covariant expansion of a general field. It can be done by taking covariant derivatives and coincidence limits of the rewritten equation (B.6)

$$
A_{\boldsymbol{\nu} \ldots}^{\boldsymbol{\mu} \ldots}(z) \iota_{\boldsymbol{\mu}}^{\boldsymbol{\alpha}}(x \mid z) \ldots \iota^{-1} \boldsymbol{\boldsymbol { \nu }}(x \mid z) \ldots=\sum_{k \in \mathbb{N}_{0}} \frac{(-1)^{k}}{k !} A_{k \boldsymbol{\beta} \ldots \boldsymbol{\mu}_{1} \ldots \boldsymbol{\mu}_{k}}^{\boldsymbol{\alpha} \ldots)} \vec{\sigma}^{\boldsymbol{\mu}_{1}}(x \mid z) \ldots \vec{\sigma}^{\boldsymbol{\mu}_{k}}(x \mid z)
$$

We will not list explicit results.

We can also expand a bi-tensor $A(x \mid z)$ in both its arguments around some point $y$. We denote coefficients of such expansion $A_{k, l}(y)$. I.e.

$$
\iota^{\star}(y \mid y ; X) \iota^{\star}(y \mid y ; Z) A(y ; X \mid y ; Z)=\sum_{k, l \in \mathbb{N}} A_{k, l} \boldsymbol{\mu}_{1} \ldots \boldsymbol{\mu}_{k} \boldsymbol{\nu}_{1} \ldots \boldsymbol{\nu}_{l}(y) X^{\boldsymbol{\mu}_{1}} \ldots X^{\boldsymbol{\mu}_{k}} Z^{\boldsymbol{\nu}_{1}} \ldots Z^{\boldsymbol{\nu}_{k}}
$$

where by $\iota^{\star}(y \mid z) A(z)$ we mean a parallel transport of all indices from $z$ to $y$. In the case of a bi-scalar $f(x \mid z)$, similarly to (B.27) we can derive that

$$
f_{k, l} \boldsymbol{\mu}_{1} \ldots \boldsymbol{\mu}_{k} \boldsymbol{\nu}_{1} \ldots \boldsymbol{\nu}_{l}=\left[\nabla_{\left(1 \boldsymbol{\mu}_{1}\right.} \ldots \nabla_{\left.1 \boldsymbol{\mu}_{k}\right)} \nabla_{\left(\mathrm{r} \boldsymbol{\nu}_{1}\right.} \ldots \nabla_{\left.\mathrm{r} \boldsymbol{\nu}_{l}\right)} f\right]
$$

For calculations in appendix $\mathrm{A}$ we need the covariant expansion of the world function $\sigma(x \mid z)$. When we expand both its arguments at point $y$ using the method described above we obtain

$$
\sigma(y ; X \mid y ; Z)=\frac{1}{2}(X-Z)^{\mu} g_{\mu \nu}(y)(X-Z)^{\nu}-\frac{1}{6} X^{\mu} X^{\nu} Z^{\kappa} Z^{\boldsymbol{\lambda}} \mathrm{R}_{\mu \kappa \nu \boldsymbol{\lambda}}(y)+\ldots .
$$

Clearly, the expansion of the world function at one of its arguments is given by equation (B.16).

Similarly, it is possible to derive (see [21, 22]) that coincidence limits of derivatives of the VanVleck Morette determinant are

$$
\begin{gathered}
{[\Delta]=1,} \\
{\left[\mathrm{~d}_{1} \Delta\right]=\left[\mathrm{d}_{\mathrm{r}} \Delta\right]=0,} \\
{\left[\nabla_{\iota_{\mu}} \nabla_{l \nu} \Delta\right]=-\left[\nabla_{\mathrm{l} \mu} \nabla_{\mathrm{r} \nu} \Delta\right]=\left[\nabla_{\mathrm{r} \mu} \nabla_{\mathrm{r} \nu} \Delta\right]=\frac{1}{3} \operatorname{Ric}_{\mu \nu} .}
\end{gathered}
$$

and the covariant expansion

$$
\Delta(y ; X \mid y ; Z)=1+\frac{1}{6}(X-Z) \cdot \operatorname{Ric} \cdot(X-Z)+\ldots .
$$


Finally let us note that the Jacobian associated with a map

$$
\boldsymbol{u}_{x}^{-1}: z \rightarrow Z=-\vec{\sigma}(x \mid z)
$$

is given by

$$
\left|\operatorname{Det} \mathrm{D} \boldsymbol{u}_{x}^{-1}(z)\right|=\left|\operatorname{Det}\left(g^{-1}(x) \cdot \stackrel{\leftrightarrow}{\sigma}(x \mid z)\right)\right|=\mathfrak{g}^{-1}(x) \mathfrak{s}(x \mid z)=\mathfrak{i}(x \mid z) \Delta(x \mid z)
$$

\section{$(d-1)+1$ splitting near a boundary}

Now we turn to investigate the domain $\Omega$ with a boundary. We will study this situation locally - i.e. we will work on a neighborhood of the boundary with topology $\mathbb{R} \times \Sigma$ where $\Sigma$ is part of boundary manifold. In such neighborhood we can perform a $(d-1)+1$ splitting which is discussed for example in [23]. It is given by a time function $t$ and time flow vector $\vec{t}$ such that $\vec{t} \cdot \mathrm{d} t=1$. We use notation of usual $3+1$ splitting of spacetime even if we do not necessarily assume that $t$ plays the role of a time coordinate. We assume that the condition $t=0$ defines the boundary and that $t>0$ inside of the domain $\Omega$. We denote $\Sigma_{t}$ hypersurfaces defined by conditions $t=$ const. We denote $n$ and $\vec{n}$ inside oriented normalized normal form and vector, $q$ orthogonal projection of the metric $g$ on the hypersurfaces $\Sigma_{t}$, and $\mathfrak{d}$ orthogonal projector to hypersurfaces $\Sigma_{t}$. I.e.

$$
g=n^{2} n n+q \quad, \quad g^{-1}=n^{-2} \vec{n} \vec{n}+q^{-1} \quad, \quad \delta=\vec{n} n+\mathfrak{d},
$$

where $q^{-1}$ is inverse of $g$ in the tangent space to the hypersurfaces. The phase factor $n$ governs the signature of the metric $g$ and the character of the hypersurfaces. We will use shorthands

$$
A_{\ldots \perp \ldots \perp \ldots}^{\ldots \ldots}=A_{\ldots \boldsymbol{\nu} \ldots}^{\ldots \boldsymbol{\beta} \ldots \ldots} \boldsymbol{\delta}_{\boldsymbol{\beta}}^{\boldsymbol{\alpha}} \vec{n}^{\nu} n_{\boldsymbol{\mu}}
$$

We also use

$$
\vec{\sigma}_{\|} \stackrel{\text { def }}{=} \vec{\sigma}_{\|\|} \quad, \quad \overleftrightarrow{\sigma}_{\|} \stackrel{\text { def }}{=} \overleftrightarrow{\sigma}_{\|\|} \quad, \quad \overleftrightarrow{\sigma}_{\|} \stackrel{\text { def }}{=} \overleftrightarrow{\sigma}_{\|\|}
$$

Decomposition of the time flow vector $\vec{t}$ defines lapse $N$ and shift $\vec{N}$

$$
\vec{t}=N \vec{n}+\vec{N} \quad, \quad \mathrm{~d} t=N n .
$$

We denote D the hypersurface gradient - an orthogonal projection of a spacetime gradient to the hypersurfaces $\Sigma_{t}$

$$
\mathrm{D} f=\mathfrak{d} \cdot \mathrm{d} f,
$$

and $\nabla$ the hypersurface connection of the metric $q$. It is related to the spacetime connection as

$$
\nabla A=\mathfrak{d}^{\star} \nabla A \quad \text { for } A \text { such that } \quad A=\mathfrak{d}^{\star} A .
$$

where by $\mathfrak{b}^{\star} A$ we mean projection of all tensor indices to the spaces tangent to the boundary. We denote by R, RIC, $\mathcal{R}$, and $\nabla^{2}$ the Riemann curvature tensor, Ricci tensor, scalar curvature and Laplace operator of the metric $q$.

The extrinsic curvature $\mathrm{K}$ is given by covariant derivative of the normal form

$$
\mathrm{K}=\mathfrak{d} \cdot \nabla n
$$

and we use shorthands

$$
\begin{gathered}
k=\mathrm{K}_{\mu \nu} g^{-1 \mu \nu} \\
\mathrm{K}^{2}=\mathrm{K}_{\kappa \mu} \mathrm{K}_{\lambda \nu} g^{-1 \kappa \lambda} g^{-1 \mu \nu} .
\end{gathered}
$$


We define the time derivative of a tensor field $A$ tangent to the hypersurfaces:

$$
A^{\prime}=\mathfrak{d}^{\star} \mathcal{L}_{\vec{t}} A \quad \text { for } A \text { such that } \quad A=\mathfrak{d}^{\star} A .
$$

Now we list a number of useful relations between spacetime quantities and "space" quantities, derivations of which are straightforward and for the case $n^{2}=-1$ can be mostly found, for example, in [23].

$$
\begin{gathered}
\vec{n} \cdot \nabla n=-\mathrm{D} \ln N \\
\nabla \cdot \vec{n}=n^{2} k \\
\vec{n} \cdot \nabla q=n(n(\mathrm{D} \ln N)+(\mathrm{D} \ln N) n) \\
\mathfrak{d}_{\boldsymbol{\gamma}}^{\mu} \nabla_{\boldsymbol{\mu}} q_{\boldsymbol{\alpha} \boldsymbol{\beta}}=-\mathrm{n}_{\boldsymbol{\alpha}} \mathrm{K}_{\boldsymbol{\beta} \boldsymbol{\gamma}}-\mathrm{n}_{\boldsymbol{\beta}} \mathrm{K}_{\boldsymbol{\alpha} \boldsymbol{\gamma}} \\
\nabla \cdot \mathfrak{d}=-n^{2} n k+\mathrm{D} \ln N \\
\mathfrak{d} \cdot(\nabla \mathfrak{d}) \cdot \mathfrak{d}=-\mathrm{K} \vec{n} \\
q^{\prime}=2 n^{2} N \mathrm{~K}+\mathcal{L}_{\vec{N}} q, \\
\mathrm{~K}^{\prime}=N(\vec{n} \cdot \nabla \mathrm{K})_{\| ! \prime}+2 n^{2} N \mathrm{~K} \cdot q^{-1} \cdot \mathrm{K}+\mathcal{L}_{\vec{N}} \mathrm{~K} \\
k^{\prime}=N \vec{n} \cdot \mathrm{d} k+\vec{N} k
\end{gathered}
$$

The curvature tensors of the spacetime metric $g$ and of the space metric $q$ are related by

$$
\begin{gathered}
\mathrm{R}_{\|\boldsymbol{\alpha}\| \boldsymbol{\beta}\|\boldsymbol{\gamma}\| \boldsymbol{\delta}}=\mathrm{R}_{\boldsymbol{\alpha} \boldsymbol{\beta} \boldsymbol{\gamma} \boldsymbol{\delta}}+n^{2}\left(\mathrm{~K}_{\boldsymbol{\alpha} \boldsymbol{\delta}} \mathrm{K}_{\boldsymbol{\beta} \boldsymbol{\gamma}}-\mathrm{K}_{\boldsymbol{\alpha} \boldsymbol{\gamma}} \mathrm{K}_{\boldsymbol{\beta} \boldsymbol{\delta}}\right) \\
\mathrm{R}_{\|\boldsymbol{\alpha}\| \boldsymbol{\beta} \boldsymbol{\gamma} \perp}=n^{2}\left(\nabla_{\boldsymbol{\alpha}} \mathrm{K}_{\boldsymbol{\beta} \boldsymbol{\gamma}}-\nabla_{\boldsymbol{\beta}} \mathrm{K}_{\boldsymbol{\alpha} \boldsymbol{\gamma}}\right), \\
\mathrm{R}_{\|\perp\| \perp}=n^{4}\left(\mathrm{~K} k-\mathrm{K} \cdot q^{-1} \cdot \mathrm{K}\right)-n^{2}\left(\nabla \nabla \ln N+(\mathrm{D} \ln N)(\mathrm{D} \ln N)+(\nabla \cdot(\vec{n} \mathrm{~K}))_{\| \Perp}\right)= \\
=n^{4} \mathrm{~K} \cdot q^{-1} \cdot \mathrm{K}-n^{2}\left(\nabla \nabla \ln N+(\mathrm{D} \ln N)(\mathrm{D} \ln N)+\frac{1}{N} \mathrm{~K}^{\prime}-\frac{1}{N} \mathcal{L}_{\vec{N}} \mathrm{~K}\right), \\
\operatorname{Ric}_{\|\|}=\mathrm{RIC}-\nabla \nabla \ln N-(\mathrm{D} \ln N)(\mathrm{D} \ln N)-(\nabla \cdot(\vec{n} \mathrm{~K}))_{\|\|}, \\
\operatorname{Ric}_{\| \perp}=n^{2}\left(\nabla \cdot q^{-1} \cdot \mathrm{K}-\mathrm{D} k\right) \quad, \\
\operatorname{Ric}_{\perp \perp}=n^{4}\left(k^{2}-\mathrm{K}^{2}\right)-n^{2} \nabla \cdot\left(\vec{n} k+q^{-1} \cdot(\mathrm{D} \ln N)\right)= \\
=-n^{4} \mathrm{~K}^{2}-n^{2}\left(\nabla^{2} \ln N+(\mathrm{D} \ln N) \cdot q^{-1} \cdot(\mathrm{D} \ln N)+\vec{n} \cdot \mathrm{d} k\right) \\
R=\mathcal{R}+n^{2}\left(k^{2}-\mathrm{K}^{2}\right)-2 \nabla \cdot\left(\vec{n} k+q^{-1} \cdot(\mathrm{D} \ln N)\right)= \\
=\mathcal{R}-n^{2}\left(k^{2}+\mathrm{K}^{2}\right)-2\left(\vec{n} \cdot \mathrm{d} k+\nabla^{2} \ln N+(\mathrm{D} \ln N) \cdot q^{-1} \cdot(\mathrm{D} \ln N)\right)
\end{gathered}
$$

\section{Geodesic theory near a boundary}

We can develop geodesic theory on a hypersurface $\Sigma$ similarly to what we did for the spacetime $M$. On the boundary we denote the exponential map $\boldsymbol{v}_{x}$, the tensor of geodesic transform and its determinant $\varsigma$ and $\mathfrak{j}$, the world function, its derivatives and its determinant $\rho, \vec{\rho}, \stackrel{\leftarrow}{\rho}, \vec{\rho}, \stackrel{\leftrightarrow}{\rho}, \stackrel{\leftarrow}{\rho}$ and $\mathfrak{r}$, and Van-Vleck Morette determinant $\triangle$. Finally, we denote $\{$.$\} the coincidence limit on the$ boundary.

In the neighborhood of a part of the boundary $\Sigma$ of the domain $\Omega$ in which geodesics orthogonal to the boundary do not cross we can also define the map $\hat{\boldsymbol{w}}$

$$
\begin{gathered}
\widehat{\boldsymbol{w}}: \Sigma \times \mathbb{R} \rightarrow M, \\
\widehat{\boldsymbol{w}}(x, \eta) \quad \text { is geodesic } \quad, \quad \widehat{\boldsymbol{w}}(x, 0)=x \quad, \quad \widehat{\boldsymbol{w}}^{\prime}(x, 0)=\vec{n} .
\end{gathered}
$$


It maps point $x$ on the boundary "orthogonally" to the domain $\Omega$ by the distance $\eta$. We denote $\Sigma_{\eta}$ the hypersurface which we obtain by shifting $\Sigma=\Sigma_{0}$ by the distance $\eta$. We also use the notation

$$
\begin{gathered}
\widehat{\boldsymbol{w}}_{\eta}: \Sigma \rightarrow \Sigma_{\eta} \quad, \quad \widehat{\boldsymbol{w}}_{\eta}(x)=\widehat{\boldsymbol{w}}(x, \eta) \\
\widehat{\boldsymbol{w}}_{\xi, \zeta}: \Sigma_{\xi} \rightarrow \Sigma_{\zeta} \quad, \quad \widehat{\boldsymbol{w}}_{\xi, \zeta}=\widehat{\boldsymbol{w}}_{\xi}\left(\widehat{\boldsymbol{w}}_{\zeta}^{-1}\right)
\end{gathered}
$$

This foliation is special case of the foliation discussed above. We obtain it for the choice of lapse and shift $N=1$ and $\vec{N}=0$.

For a tensor field $A(x)$ on spacetime we denote by $A(\widehat{x}, \xi)$ its dependence on $\widehat{x}$ and $\xi$, and $\hat{A}(\widehat{x}, \xi)$ the tensor field on the boundary manifold obtained by transformation of tensor indices of $A(\widehat{x}, \xi)$ to the boundary tangent bundle using $\widehat{\boldsymbol{w}}_{-\xi}^{\star}$

$$
A(\widehat{x}, \xi)=A(\widehat{\boldsymbol{w}}(\widehat{x}, \xi)) \quad, \quad \hat{A}(\widehat{x}, \xi)=\widehat{\boldsymbol{w}}_{-\xi}^{\star} A\left(\widehat{\boldsymbol{w}}_{\xi}(\widehat{x})\right) \quad,
$$

where $\widehat{\boldsymbol{w}}_{\xi}^{\star}$ is the induced transformation on tangent bundles. For a bi-tensor $A(x \mid z)$ we mean by the boundary coincidence limit

$$
\{A\}(\widehat{y} ; \xi, \zeta)=A(\widehat{y}, \xi \mid \widehat{y}, \zeta)
$$

Specially, we have a metric $\widehat{q}(\widehat{y}, \eta)$ (generally different from $q(\widehat{y})$ ) on the boundary manifold, volume element $\mathfrak{q}^{\frac{1}{2}}(\hat{y}, \eta)$, and associated connection $\widehat{\nabla}$. It is related to the connection $\nabla$ by

$$
\widehat{\nabla}=\nabla \oplus \hat{\gamma}
$$

The relation of corresponding curvature tensors is (see e.g. [23])

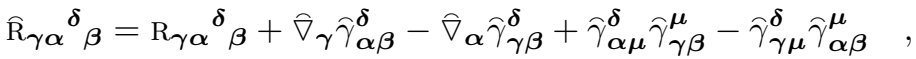

$$
\begin{aligned}
& \mathrm{RIC}_{\boldsymbol{\alpha} \boldsymbol{\beta}}=\mathrm{RIC}_{\boldsymbol{\alpha} \boldsymbol{\beta}}+\widehat{\nabla}_{\boldsymbol{\mu}} \widehat{\gamma}_{\boldsymbol{\alpha} \boldsymbol{\beta}}^{\boldsymbol{\mu}}-\widehat{\nabla}_{\boldsymbol{\alpha}} \hat{\gamma}_{\boldsymbol{\beta} \boldsymbol{\mu}}^{\boldsymbol{\mu}}+\widehat{\gamma}_{\boldsymbol{\alpha} \boldsymbol{\mu}}^{\nu} \hat{\gamma}_{\boldsymbol{\beta} \boldsymbol{\nu}}^{\boldsymbol{\mu}}-\widehat{\gamma}_{\boldsymbol{\mu} \nu}^{\nu} \hat{\gamma}_{\boldsymbol{\alpha} \boldsymbol{\beta}}^{\boldsymbol{\mu}} \text {. }
\end{aligned}
$$

From the definition of the map $\widehat{\boldsymbol{w}}$ we have

$$
\begin{gathered}
\{\vec{\sigma}\}(\widehat{y} ; \xi, \zeta)=(\xi-\zeta) \vec{n}(\widehat{y}, \xi) \\
\left\{\mathrm{D}_{1} \sigma\right\}=0
\end{gathered}
$$

Differentiating this equation we obtain the differential map D $\hat{\boldsymbol{w}}$

$$
\begin{aligned}
& \mathrm{D} \widehat{\boldsymbol{w}}_{\xi, \zeta}(x): \mathbf{T}_{x} \Sigma_{\xi} \rightarrow \mathbf{T}_{z} \Sigma_{\zeta} \quad, \quad z=\widehat{\boldsymbol{w}}_{\xi, \zeta}(x), \\
& \mathrm{D}_{\boldsymbol{\mu}}^{\nu} \widehat{\boldsymbol{w}}_{\xi, \zeta}(x)=-\left(\nabla_{1 \boldsymbol{\mu}} \nabla_{1 \boldsymbol{\kappa}} \sigma\right)(x \mid z) \stackrel{\leftrightarrow}{\sigma}_{\|}^{-1} \boldsymbol{\kappa} \boldsymbol{\nu}(x \mid z)
\end{aligned}
$$

In the special case $\xi=0$ we get

$$
\mathrm{D}_{\boldsymbol{\mu}}^{\boldsymbol{\nu}} \widehat{\boldsymbol{w}}_{\eta}(\widehat{y})=-\left(\eta n^{2} \mathrm{~K}_{\boldsymbol{\mu} \kappa}(\widehat{y})+\vec{\sigma}_{\| \boldsymbol{\mu} \kappa}(\widehat{y} \mid \widehat{y}, \eta)\right) \stackrel{\leftrightarrow}{\sigma}_{\|}^{-1} \boldsymbol{\kappa} \boldsymbol{\nu}(\widehat{y} \mid \widehat{y}, \eta)
$$

Here $\stackrel{\leftrightarrow}{\sigma}_{\|}^{-1}$ is the inverse of $\stackrel{\leftrightarrow}{\sigma}_{\|}$in spaces tangent to hypersurfaces $\Sigma_{\eta}$. Because $\mathrm{D} \widehat{\boldsymbol{w}}_{\xi, \zeta}=\mathrm{D} \widehat{\boldsymbol{w}}_{\zeta, \xi}^{-1}$ we have

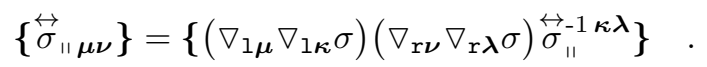

Using this relation, the definition of the Van-Vleck Morette determinant and

$$
\vec{n}(x) \cdot \stackrel{\leftrightarrow}{\sigma}(x \mid z)=-\vec{n}(z) \cdot g(z) \quad \text { for } \quad z=\widehat{\boldsymbol{w}}_{\xi, \zeta}(x)
$$


we get an expression for the Jacobian associated with the map $\widehat{\boldsymbol{w}}_{\xi, \zeta}$,

$$
\begin{aligned}
\hat{j}(\widehat{y} ; \xi, \zeta) & =\left|\operatorname{Det}_{\| 1} \operatorname{D}_{\xi, \zeta}\right|(\widehat{y}, \xi)= \\
& =\left\{\Delta^{-1} \mathfrak{q}^{-1}\left(\operatorname{Det}_{\|} \nabla_{\mathfrak{l}} \nabla_{\mathbf{l}} \sigma\right)\right\}(\widehat{y} ; \xi, \zeta)=\left\{\Delta \mathfrak{q}\left(\operatorname{Det}_{\|} \nabla_{\mathbf{r}} \nabla_{\mathbf{r}} \sigma\right)^{-1}\right\}(\widehat{y} ; \xi, \zeta)
\end{aligned}
$$

As special cases we have

$$
\hat{j}(\widehat{y}, \eta)=\hat{j}(\widehat{y} ; 0, \eta) \quad, \quad \hat{j}(\widehat{y} ; \xi, \zeta)=\hat{j}^{-1}(\widehat{y}, \xi) \hat{j}(\widehat{y}, \zeta) \quad .
$$

This also gives the expression for the Van-Vleck Morette determinant,

$$
\Delta(\widehat{y}, \xi \mid \widehat{y}, \zeta)=\mathfrak{q}^{-\frac{1}{2}}(\widehat{y}, \xi) \mathfrak{q}^{-\frac{1}{2}}(\widehat{y}, \zeta)\left(\left(\operatorname{Det}_{\|} \nabla_{\mathbf{l}} \nabla_{\mathbf{l}} \sigma\right)\left(\operatorname{Det}_{\|} \nabla_{\mathbf{r}} \nabla_{\mathbf{r}} \sigma\right)\right)^{\frac{1}{2}}(\widehat{y}, \xi \mid \widehat{y}, \zeta)
$$

Finally we can prove that

$$
\begin{aligned}
& \hat{j}(\widehat{y}, \xi) \hat{\Delta}(\widehat{y}, \xi \mid \widehat{y}, \zeta) \hat{j}(\widehat{y}, \zeta)= \\
& =\mathfrak{q}^{-1}(\widehat{y})\left(\operatorname{Det}_{\|} \nabla_{\mathfrak{l}} \nabla_{\mathbf{l}} \widehat{\sigma}\right)(\widehat{y}, \xi \mid \widehat{y}, \zeta)=\mathfrak{q}^{-1}(\widehat{y})\left(\operatorname{Det}_{\|} \nabla_{\mathbf{r}} \nabla_{\mathbf{r}} \widehat{\sigma}\right)(\widehat{y}, \xi \mid \widehat{y}, \zeta) .
\end{aligned}
$$

Thanks to (B.67), (B.72), and (B.73), the function $\tilde{j}$ defined in $(\mathrm{A} .36)$ is

$$
\begin{aligned}
\tilde{j}(\widehat{y} ; \xi, \zeta) & =\hat{j}(\widehat{y}, \xi) \widehat{\Delta}(\widehat{y}, \xi \mid \widehat{y}, \zeta) \hat{j}(\widehat{y}, \zeta) \mathfrak{q}^{\frac{1}{2}}(\widehat{y})\left(\operatorname{Det}_{\|} \nabla_{\mathbf{r}} \nabla_{\mathbf{r}} \widehat{\sigma}\right)^{-\frac{1}{2}}(\widehat{y}, \xi \mid \widehat{y}, \zeta)= \\
& =\hat{j}(\widehat{y}, \xi) \widehat{\Delta}(\hat{y}, \xi \mid \widehat{y}, \zeta) \mathfrak{q}^{\frac{1}{2}}(\widehat{y}, \zeta)\left(\operatorname{Det}_{\|} \widehat{\nabla}_{\mathbf{r}} \widehat{\nabla}_{\mathbf{r}} \widehat{\sigma}\right)^{-\frac{1}{2}}(\widehat{y}, \xi \mid \widehat{y}, \zeta)= \\
& =j(x) \Delta(x \mid z) \mathfrak{q}^{\frac{1}{2}}(z)\left(\operatorname{Det}_{\|} \nabla_{\mathbf{r}} \nabla_{\mathbf{r}} \sigma\right)^{-\frac{1}{2}}(x \mid z)=j(x) \Delta^{\frac{1}{2}}(x \mid z) \hat{j}(\widehat{y} ; \xi, \zeta)^{\frac{1}{2}}= \\
& =(\hat{j}(\widehat{y}, \xi) \hat{\Delta}(\widehat{y}, \xi \mid \widehat{y}, \zeta) \hat{j}(\widehat{y}, \zeta))^{\frac{1}{2}} .
\end{aligned}
$$

The equation previous to this one is a straightforward consequence.

\section{Reflection on the boundary}

In section 2 we have worked with the geodesic reflected on the boundary. We recall its definition here and list some useful properties which allows us to prove the relation (A.52).

We will study the geodesic $\overline{\boldsymbol{x}}_{\mathrm{b}}(x \mid z)$ between points $x$ and $z$ which is reflecting on the boundary at point $b(x \mid z)$ - an extreme trajectory of the functional given by the half of squared length, with the condition that it has to touch the boundary. We use the convention, that for any quantity depending on two spacetime points $f(x \mid z)$ we denote

$$
f_{1}(x \mid z)=f(x \mid b(x \mid z)) \quad, \quad f_{\mathrm{r}}(x \mid z)=f(b(x \mid z) \mid z) .
$$

If we denote the parameter at which the geodesic reflects on the boundary $\lambda_{\mathrm{r}}(x \mid z)$ and its complement $\lambda_{1}(x \mid z)$

$$
\begin{gathered}
b(x \mid z)=\left.\overline{\boldsymbol{x}}_{\mathrm{b}}(x \mid z)\right|_{\lambda_{\mathrm{r}}(x \mid z)} \in \partial \Omega, \\
1=\lambda_{\mathrm{l}}(x \mid z)+\lambda_{\mathrm{r}}(x \mid z)
\end{gathered}
$$

we can write the reflected geodesic as joining of two geodesics

$$
\left[\tau, \overline{\boldsymbol{x}}_{\mathrm{b}}\right]=\left[\lambda_{\mathrm{l}} \tau, \overline{\boldsymbol{x}}_{1}\right] \odot\left[\lambda_{\mathrm{r}} \tau, \overline{\boldsymbol{x}}_{\mathrm{r}}\right] .
$$


The extremum conditions on position of the reflection point and reflection parameter are

$$
\frac{(\mathrm{D} \sigma)_{1}}{\lambda_{\mathrm{l}}}+\frac{(\mathrm{D} \sigma)_{\mathrm{r}}}{\lambda_{\mathrm{r}}}=0 \quad, \quad \frac{\sigma_{\mathrm{l}}}{\lambda_{\mathrm{l}}^{2}}=\frac{\sigma_{\mathrm{r}}}{\lambda_{\mathrm{r}}^{2}},
$$

where $\mathrm{D}$ acts in the argument on the boundary.

We define reflection world function $\sigma_{\mathrm{b}}$ as

$$
\sigma_{\mathrm{b}}=\frac{\sigma_{\mathrm{l}}}{\lambda_{\mathrm{l}}}+\frac{\sigma_{\mathrm{r}}}{\lambda_{\mathrm{r}}}=\frac{\sigma_{1}}{\lambda_{\mathrm{l}}^{2}}=\frac{\sigma_{\mathrm{r}}}{\lambda_{\mathrm{r}}^{2}}
$$

Clearly

$$
\begin{gathered}
\lambda_{\mathrm{l}}=\sqrt{\frac{\sigma_{\mathrm{l}}}{\sigma_{\mathrm{b}}}}, \quad \lambda_{\mathrm{r}}=\sqrt{\frac{\sigma_{\mathrm{r}}}{\sigma_{\mathrm{b}}}}, \\
\sqrt{\sigma_{\mathrm{b}}}=\sqrt{\sigma_{\mathrm{l}}}+\sqrt{\sigma_{\mathrm{r}}} \quad, \quad 0=(\mathrm{D} \sqrt{\sigma})_{\mathrm{l}}+(\mathrm{D} \sqrt{\sigma})_{\mathrm{r}},
\end{gathered}
$$

Using the last equation we can get

$$
\mathrm{d}_{\mathrm{l}} \sqrt{\sigma_{\mathrm{b}}}=\left(\mathrm{d}_{\mathrm{l}} \sqrt{\sigma}\right)_{\mathrm{l}} \quad, \quad \mathrm{d}_{\mathrm{r}} \sqrt{\sigma_{\mathrm{b}}}=\left(\mathrm{d}_{\mathrm{r}} \sqrt{\sigma}\right)_{\mathrm{r}} .
$$

Similarly to the case without boundary we define

$$
\begin{gathered}
s_{\mathrm{b}}=\left|\sqrt{2 \sigma_{\mathrm{b}}}\right|=s_{1}+s_{\mathrm{r}} \\
\vec{\sigma}_{\mathrm{b}}=g^{-1} \cdot \mathrm{d}_{\mathrm{l}} \sigma_{\mathrm{b}}=\frac{\vec{\sigma}_{1}}{\lambda_{\mathrm{l}}} \quad, \quad \overleftarrow{\sigma}_{\mathrm{b}}=\mathrm{d}_{\mathrm{r}} \sigma_{\mathrm{b}} \cdot g^{-1}=\frac{\overleftarrow{\sigma}_{\mathrm{r}}}{\lambda_{\mathrm{r}}} \\
\vec{\sigma}_{\mathrm{b}}=\nabla_{1} \nabla_{\mathrm{l}} \sigma_{\mathrm{b}} \quad, \quad \stackrel{\leftrightarrow}{\sigma}_{\mathrm{b}}=\mathrm{d}_{\mathrm{l}} \mathrm{d}_{\mathrm{r}} \sigma_{\mathrm{b}} \quad, \quad \overleftarrow{\sigma}_{\mathrm{b}}=\nabla_{\mathrm{r}} \nabla_{\mathrm{r}} \sigma_{\mathrm{b}}
\end{gathered}
$$

Additionally we define

$$
s_{\perp}=-\frac{1}{\lambda_{\mathrm{l}}} \vec{n}(b) \cdot\left(\mathrm{d}_{\mathrm{r}} \sigma\right)_{\mathrm{l}}=-\frac{1}{\lambda_{\mathrm{r}}} \vec{n}(b) \cdot\left(\mathrm{d}_{\mathrm{l}} \sigma\right)_{\mathrm{r}}=\vec{n} \cdot\left(\mathrm{d}_{\mathrm{l}} \sigma_{\mathrm{b}}\right)=\vec{n} \cdot\left(\mathrm{d}_{\mathrm{r}} \sigma_{\mathrm{b}}\right),
$$

and we denote differentials of maps $x \rightarrow b(x \mid z)$ and $z \rightarrow b(x \mid z)$ as

$$
\vec{b}=\mathrm{D}_{1} b \quad, \quad \overleftarrow{b}=\mathrm{D}_{\mathrm{r}} b
$$

i.e. if we displace points $x$ and $z$ in directions $X$ and $Z$, the reflection point moves in direction $X \cdot b(x \mid z)+b(x \mid z) \cdot Z$. Finally we define the reflection Van-Vleck Morette determinant $\Delta_{\mathrm{b}}$

$$
\Delta_{\mathrm{b}}=\left|\operatorname{Det} \stackrel{\leftrightarrow}{\sigma}_{\mathrm{b}}\right| \underline{\mathfrak{i}}^{-1}
$$

Some long algebra gives

$$
\begin{aligned}
& \vec{\sigma}_{\mathrm{b}}=-\vec{b} \cdot B \cdot \vec{b}-\frac{1}{2 \sigma_{\mathrm{b}}} \frac{\lambda_{\mathrm{r}}}{\lambda_{\mathrm{l}}}\left(\mathrm{d}_{\mathrm{l}} \sigma_{\mathrm{b}}\right)\left(\mathrm{d}_{\mathrm{l}} \sigma_{\mathrm{b}}\right)+\frac{1}{\lambda_{\mathrm{l}}} \vec{\sigma}_{\mathrm{l}}, \\
& \overleftrightarrow{\sigma}_{\mathrm{b}}=-\vec{b} \cdot B \cdot \overleftarrow{b}+\frac{1}{2 \sigma_{\mathrm{b}}}\left(\mathrm{d}_{\mathrm{l}} \sigma_{\mathrm{b}}\right)\left(\mathrm{d}_{\mathrm{r}} \sigma_{\mathrm{b}}\right), \\
& \overleftarrow{\sigma}_{\mathrm{b}}=-\overleftarrow{b} \cdot B \cdot \overleftarrow{b}-\frac{1}{2 \sigma_{\mathrm{b}}} \frac{\lambda_{\mathrm{l}}}{\lambda_{\mathrm{r}}}\left(\mathrm{d}_{\mathrm{r}} \sigma_{\mathrm{b}}\right)\left(\mathrm{d}_{\mathrm{r}} \sigma_{\mathrm{b}}\right)+\frac{1}{\lambda_{\mathrm{r}}} \overleftarrow{\sigma}_{\mathrm{l}},
\end{aligned}
$$

where

$$
\left.\left.B=2 \sqrt{\sigma_{\mathrm{b}}}\left(\nabla_{\mathrm{r}} \nabla_{\mathrm{r}} \sqrt{\sigma}\right)_{1}+\nabla_{\mathrm{l}} \nabla_{\mathrm{l}} \sqrt{\sigma}\right)_{\mathrm{r}}\right)=\frac{\overleftarrow{\sigma}_{\|} \mathrm{l}}{\lambda_{\mathrm{l}}}+\frac{\vec{\sigma}_{\| \mathrm{r}}}{\lambda_{\mathrm{r}}}+\frac{1}{2} \frac{\sigma_{\mathrm{b}}}{\sigma_{\mathrm{l}} \sigma_{\mathrm{r}}}\left(\mathrm{D}_{1} \sigma\right)_{\mathrm{r}}\left(\mathrm{D}_{\mathrm{r}} \sigma\right)_{1}+2 s_{\perp} \mathrm{K}(b) .
$$


Using these relations, a more intricate calculation gives the space coincidence limits

$$
\begin{aligned}
& \left\{\stackrel{\leftrightarrow}{\sigma}_{\mathrm{bII}}\right\}=-\{\vec{b} \cdot B \cdot \overleftarrow{b}\} \\
& \left\{\frac{\overleftrightarrow{\sigma}_{\| 1}}{\lambda_{1}}\right\}=-\{\vec{b} \cdot B\} \quad, \quad\left\{\frac{\overleftrightarrow{\sigma}_{\| \mathrm{r}}}{\lambda_{\mathrm{r}}}\right\}=-\{B \cdot \overleftarrow{b}\}, \\
& \left\{\left(\nabla_{\mathrm{l}} \nabla_{\mathrm{l}} \sigma_{\mathrm{b}}\right)^{-1}\right\}=\left\{\stackrel{\leftrightarrow}{\sigma_{\text {bII }}^{-1}} \cdot\left(\nabla_{\mathrm{r}} \nabla_{\mathrm{r}} \sigma_{\mathrm{b}}\right)^{-1} \cdot \stackrel{\leftrightarrow}{\sigma}_{\text {bll }}^{-1}\right\}= \\
& =\left\{\stackrel{\leftrightarrow}{\sigma}_{\| 1}^{-1} \cdot\left(\nabla_{\mathrm{r}} \nabla_{\mathrm{r}} \sigma\right)_{1} \cdot\left(\lambda_{\mathrm{l}}\left(\nabla_{\mathrm{r}} \nabla_{\mathrm{r}} \sigma\right)_{1}^{-1}+\lambda_{\mathrm{r}}\left(\nabla_{\mathrm{l}} \nabla_{\mathbf{l}} \sigma\right)_{\mathrm{r}}^{-1}\right) \cdot\left(\nabla_{\mathrm{r}} \nabla_{\mathrm{r}} \sigma\right)_{1} \cdot \stackrel{\leftrightarrow}{\sigma}_{\| 1}^{-1}\right\}, \\
& \left\{\left(\nabla_{\mathrm{l}} \nabla_{\mathrm{l}} \sigma_{\mathrm{b}}\right)^{-1} \cdot \stackrel{\leftrightarrow}{\sigma}_{\mathrm{bII}}\right\}=\left\{\stackrel{\leftrightarrow}{\sigma}_{\mathrm{bII}}^{-1} \cdot\left(\nabla_{\mathrm{r}} \nabla_{\mathrm{r}} \sigma_{\mathrm{b}}\right)\right\}
\end{aligned}
$$

Here inverses are taken in the spaces tangent to the boundary. Taking the determinant of the last equation, we find

$$
\begin{aligned}
& \left\{\left(\text { Det }_{\|} \stackrel{\leftrightarrow}{\sigma}_{\text {bII }}\right)^{2}\right\}=\left\{\left(\text { Det }_{\|} \nabla_{1} \nabla_{1} \sigma_{\mathrm{b}}\right)\left(\text { Det }_{\|} \nabla_{\mathrm{r}} \nabla_{\mathrm{r}} \sigma_{\mathrm{b}}\right)\right\} \\
& \Delta_{\mathrm{b}}(\widehat{y}, \xi \mid \widehat{y}, \zeta)=\left(\mathfrak{q}^{-\frac{1}{2}}(\widehat{y}, \xi) \mathfrak{q}^{-\frac{1}{2}}(\widehat{y}, \zeta)\left(\operatorname{Det}_{\|} \nabla_{1} \nabla_{\mathrm{l}} \sigma_{\mathrm{b}}\right)\left(\operatorname{Det}_{\|} \nabla_{\mathrm{r}} \nabla_{\mathrm{r}} \sigma_{\mathrm{b}}\right)\right)^{\frac{1}{2}}(\widehat{y}, \xi \mid \widehat{y}, \zeta)
\end{aligned}
$$

and

$$
\begin{gathered}
\frac{j^{2}(x)}{\mathfrak{q}(x)}\left(\operatorname{Det}_{\|} \nabla_{\mathfrak{l}} \nabla_{\mathfrak{l}} \sigma_{\mathrm{b}}\right)(x \mid z)=\frac{j^{2}(z)}{\mathfrak{q}(z)}\left(\operatorname{Det}_{\|} \nabla_{\mathrm{r}} \nabla_{\mathrm{r}} \sigma_{\mathrm{b}}\right)(x \mid z)= \\
=\frac{j^{2}(\widehat{y})}{\mathfrak{q}(\widehat{y})}\left(\lambda_{\mathrm{l}}\left(\nabla_{\mathrm{r}} \nabla_{\mathrm{r}} \sigma\right)_{\mathrm{l}}^{-1}+\lambda_{\mathrm{r}}\left(\nabla_{\mathfrak{l}} \nabla_{\mathfrak{l}} \sigma\right)_{\mathrm{r}}^{-1}\right)(x \mid z)
\end{gathered}
$$

for $x=\widehat{\boldsymbol{w}}(\widehat{y}, \xi)$ and $z=\widehat{\boldsymbol{w}}(\widehat{y}, \zeta)$. Putting these relation together we obtain

$$
j(x) \Delta_{\mathrm{b}}(x \mid z) j(z)=\frac{j^{2}(x)}{\mathfrak{q}(x)}\left(\operatorname{Det}_{\|} \nabla_{\mathfrak{l}} \nabla_{\mathfrak{l}} \sigma_{\mathrm{b}}\right)(x \mid z)=\frac{j^{2}(z)}{\mathfrak{q}(z)}\left(\operatorname{Det}_{\|} \nabla_{\mathbf{r}} \nabla_{\mathbf{r}} \sigma_{\mathrm{b}}\right)(x \mid z)
$$

for $x=\widehat{\boldsymbol{w}}(\widehat{y}, \xi)$ and $z=\widehat{\boldsymbol{w}}(\widehat{y}, \zeta)$. The equality in (A.52) is a straightforward consequence of this relation.

\section{Covariant expansions near boundary}

Finally we will write down coefficients in covariant expansions (A.32) and (A.33) of the world function $\sigma$ and function $l$ defined in (A.30). These are expansions inside of the boundary manifold of $\widehat{\boldsymbol{w}}$-mapped functions $\widehat{\sigma}(\widehat{x}, \xi \mid \bar{z}, \zeta)$ and $\widehat{l}(\widehat{x}, \xi \mid \bar{z}, \zeta)$ around point $\widehat{x}$. The derivation is long and technical. It uses the general method discussed above and a transformation of the connection $\nabla$ to the connection $\vec{\nabla}$. Fortunately, we need only the spacetime coincidence limit of the coefficients (i.e. $\left.\widehat{\sigma}_{k, l}(\widehat{y} ; \eta, \eta)\right)$, which simplifies the calculations significantly. But even then the calculations is too long and uninteresting to be included it here. We list only the results. See also [18 20] for similar calculations.

The coefficients of the boundary covariant expansion of the spacetime world function $\widehat{\sigma}$ at some general point $\hat{y}$ (slight generalization of eq. (A.32)) are

$$
\begin{aligned}
& \widehat{\sigma}_{0,0}(\hat{y} ; \xi, \zeta)=\frac{1}{2} n^{2}(\xi-\zeta)^{2}, \\
& \widehat{\sigma}_{0,1}(\hat{y} ; \xi, \zeta)=\widehat{\sigma}_{1,0}(\hat{y} ; \xi, \zeta)=0 \quad \text {, } \\
& {\left[\widehat{\sigma}_{2,0}\right]=-\left[\widehat{\sigma}_{1,1}\right]=\left[\widehat{\sigma}_{0,2}\right]=\widehat{q},} \\
& {\left[\widehat{\sigma}_{3,0 \boldsymbol{\alpha} \boldsymbol{\beta} \gamma}\right]=\left[\widehat{\sigma}_{0,3 \boldsymbol{\alpha} \boldsymbol{\beta} \gamma}\right]=3 \hat{\gamma}_{(\boldsymbol{\alpha} \boldsymbol{\beta}}^{\boldsymbol{\mu}} \widehat{q}_{\boldsymbol{\gamma}) \boldsymbol{\mu}} \quad, \quad\left[\widehat{\sigma}_{2,1 \boldsymbol{\alpha} \boldsymbol{\beta} \boldsymbol{\kappa}}\right]=\left[\widehat{\sigma}_{1,2 \boldsymbol{\kappa} \boldsymbol{\alpha} \boldsymbol{\beta}}\right]=-\widehat{\gamma}_{\boldsymbol{\alpha} \boldsymbol{\beta}}^{\boldsymbol{\mu}} \widehat{q}_{\boldsymbol{\kappa} \boldsymbol{\mu}} \quad,}
\end{aligned}
$$




$$
\begin{aligned}
& {\left[\widehat{\sigma}_{4,0 \boldsymbol{\alpha} \boldsymbol{\beta} \gamma \boldsymbol{\delta}}\right]=\left[\widehat{\sigma}_{0,4 \boldsymbol{\alpha} \boldsymbol{\beta} \gamma \delta}\right]=} \\
& =-n^{2} \widehat{\mathrm{K}}_{(\boldsymbol{\alpha} \boldsymbol{\beta}} \widehat{\mathrm{K}}_{\boldsymbol{\gamma} \boldsymbol{\delta})}+4\left(\widehat{\nabla}_{(\boldsymbol{\alpha}} \hat{\gamma}_{\boldsymbol{\beta} \boldsymbol{\gamma}}^{\boldsymbol{\mu}}\right) \widehat{q}_{\boldsymbol{\delta}) \boldsymbol{\mu}}+8 \widehat{\gamma}_{\boldsymbol{\mu}(\boldsymbol{\alpha}}^{\nu} \widehat{\gamma}_{\boldsymbol{\beta} \boldsymbol{\gamma}}^{\boldsymbol{\mu}} \widehat{q}_{\boldsymbol{\delta}) \boldsymbol{\nu}}+3 \widehat{\gamma}_{(\boldsymbol{\alpha} \boldsymbol{\beta}}^{\boldsymbol{\mu}} \hat{\gamma}_{\boldsymbol{\gamma} \boldsymbol{\delta})}^{\boldsymbol{\nu}} \widehat{q}_{\boldsymbol{\mu \nu}}, \\
& {\left[\widehat{\sigma}_{3,1 \boldsymbol{\alpha} \boldsymbol{\beta} \gamma \boldsymbol{\kappa}}\right]=\left[\widehat{\sigma}_{1,3 \boldsymbol{\kappa} \boldsymbol{\alpha} \boldsymbol{\beta} \gamma}\right]=} \\
& =n^{2} \widehat{\mathrm{K}}_{(\boldsymbol{\alpha} \boldsymbol{\beta}} \hat{\mathrm{K}}_{\boldsymbol{\gamma}) \boldsymbol{\kappa}}-\left(\widehat{\nabla}_{(\boldsymbol{\alpha}} \hat{\gamma}_{\boldsymbol{\beta} \boldsymbol{\gamma})}^{\boldsymbol{\mu}}\right) \hat{q}_{\boldsymbol{\mu} \kappa}-\hat{\gamma}_{(\boldsymbol{\alpha} \boldsymbol{\beta}}^{\boldsymbol{\mu}} \hat{\gamma}_{\boldsymbol{\gamma}) \boldsymbol{\mu}}^{\boldsymbol{\nu}} \widehat{q}_{\boldsymbol{\nu} \kappa}, \\
& {\left[\widehat{\sigma}_{2,2 \boldsymbol{\alpha} \boldsymbol{\beta} \kappa \boldsymbol{\lambda}}\right]=-\frac{1}{3}\left(\mathrm{R}_{\boldsymbol{\alpha} \kappa \boldsymbol{\beta} \boldsymbol{\lambda}}+\mathrm{R}_{\boldsymbol{\alpha} \boldsymbol{\lambda} \boldsymbol{\beta} \kappa}\right)-} \\
& -\frac{1}{3} n^{2} \widehat{\mathrm{K}}_{\boldsymbol{\alpha} \kappa} \widehat{\mathrm{K}}_{\boldsymbol{\beta} \boldsymbol{\lambda}}-\frac{1}{3} n^{2} \widehat{\mathrm{K}}_{\boldsymbol{\alpha} \boldsymbol{\lambda}} \widehat{\mathrm{K}}_{\boldsymbol{\beta} \boldsymbol{\kappa}}-n^{2} \widehat{\mathrm{K}}_{\boldsymbol{\alpha} \boldsymbol{\beta}} \widehat{\mathrm{K}}_{\boldsymbol{\kappa} \boldsymbol{\lambda}}-\widehat{\gamma}_{\boldsymbol{\alpha} \boldsymbol{\beta}}^{\boldsymbol{\mu}} \widehat{\gamma}_{\boldsymbol{\kappa} \boldsymbol{\lambda}}^{\boldsymbol{\nu}} \widehat{q}_{\boldsymbol{\mu} \boldsymbol{\nu}} .
\end{aligned}
$$

The coefficients of the boundary covariant expansion of the function $l$ at some general point $\hat{y}$ (slight generalization of eq. (A.33)) are

$$
\begin{aligned}
& {\left[\widehat{l}_{0,0}\right]=2 \ln \hat{j} \quad,} \\
& {\left[\widehat{l}_{1,0 \boldsymbol{\alpha}}\right]=\left[\widehat{l}_{0,1 \boldsymbol{\alpha}}\right]=\hat{\gamma}_{\boldsymbol{\alpha} \boldsymbol{\mu}}^{\boldsymbol{\mu}},} \\
& {\left[\widehat{l}_{2,0 \boldsymbol{\alpha} \boldsymbol{\beta}}\right]=\left[\widehat{l}_{0,2 \boldsymbol{\alpha} \boldsymbol{\beta}}\right]=} \\
& =\frac{1}{3}\left(-\widehat{\mathrm{K}}_{\boldsymbol{\alpha} \boldsymbol{\beta}}^{\prime}+2 n^{2} \widehat{\mathrm{K}}_{\boldsymbol{\alpha} \boldsymbol{\mu}} \widehat{\mathrm{K}}_{\boldsymbol{\beta} \boldsymbol{\nu}} \widehat{q}^{-1 \mu \nu}-n^{2} \widehat{\mathrm{K}}_{\boldsymbol{\alpha} \boldsymbol{\beta}} \widehat{k}+\right. \\
& \left.+3 \widehat{\nabla}_{(\boldsymbol{\mu}} \widehat{\gamma}_{\boldsymbol{\alpha} \boldsymbol{\beta})}^{\boldsymbol{\mu}}+\widehat{\gamma}_{\boldsymbol{\alpha} \nu}^{\boldsymbol{\mu}} \widehat{\gamma}_{\boldsymbol{\beta} \boldsymbol{\mu}}^{\boldsymbol{\nu}}+2 \widehat{\gamma}_{\boldsymbol{\alpha} \boldsymbol{\beta}}^{\boldsymbol{\mu}} \widehat{\gamma}_{\boldsymbol{\mu \nu}}^{\boldsymbol{\nu}}\right), \\
& {\left[\widehat{l}_{1,1 \boldsymbol{\alpha} \boldsymbol{\beta}}\right]=\frac{1}{3}\left(\widehat{\mathrm{K}}_{\boldsymbol{\alpha} \boldsymbol{\beta}}^{\prime}-2 n^{2} \widehat{\mathrm{K}}_{\boldsymbol{\alpha} \boldsymbol{\mu}} \widehat{\mathrm{K}}_{\boldsymbol{\beta} \boldsymbol{\nu}} \widehat{q}^{-1 \mu \nu}+n^{2} \widehat{\mathrm{K}}_{\boldsymbol{\alpha} \boldsymbol{\beta}} \widehat{k}\right)}
\end{aligned}
$$

Computing normal derivatives we also get

$$
\begin{aligned}
& \frac{1}{n}(\ln \hat{j})^{\prime}=n \widehat{k}, \\
& \frac{1}{n^{2}}(\ln \hat{j})^{\prime \prime}=\widehat{k}^{\prime},
\end{aligned}
$$

and

$$
\begin{gathered}
\frac{1}{n}\left[(\ln \hat{j})^{\mathrm{r} \prime}\right]=0 \\
\frac{1}{n^{2}}\left[(\ln \hat{j})^{\mathrm{r} \mathbf{r} \prime}\right]=-\frac{1}{3}\left(\widehat{k}^{\prime}+n^{2} \widehat{\mathrm{K}}^{2}\right) .
\end{gathered}
$$

Finally, we have boundary coincidence limits

$$
\begin{array}{rll}
\left\{\sigma_{\mathrm{b}}\right\}(\widehat{y} ; \xi, \zeta)=\frac{1}{2} n^{2}(\xi+\zeta)^{2} & , & \left\{s_{\perp}\right\}(\widehat{y} ; \xi, \zeta)=n^{2}(\xi+\zeta) \\
\left\{\vec{\sigma}_{\mathrm{b}}\right\}(\widehat{y} ; \xi, \zeta)=(\xi+\zeta) \vec{n}(\widehat{y}, \xi) & , & \left\{\overleftarrow{\sigma}_{\mathrm{b}}\right\}(\widehat{y} ; \xi, \zeta)=(\xi+\zeta) \vec{n}(\widehat{y}, \zeta) \\
\left\{\lambda_{\mathrm{l}}\right\}(\widehat{y} ; \xi, \zeta)=\frac{\xi}{\xi+\zeta} & , & \left\{\lambda_{\mathrm{r}}\right\}(\widehat{y} ; \xi, \zeta)=\frac{\zeta}{\xi+\zeta}
\end{array}
$$

Using these relations, equations (B.92), (B.93), with help of (B.69) and

$$
\left\{\widehat{\vec{\sigma}}_{\| \mathrm{r}}\right\}(\widehat{y} ; \xi, \zeta)=q(\widehat{y})+\mathcal{O}\left(\zeta^{2}\right) \quad, \quad\left\{\widetilde{\widetilde{\sigma}}_{\| 1}\right\}(\widehat{y} ; \xi, \zeta)=q(\widehat{y})+\mathcal{O}\left(\xi^{2}\right)
$$

we can derive

$$
-\left\{\stackrel{\leftrightarrow}{\sigma}_{\mathrm{b} I I}\right\}(\widehat{y} ; \xi, \zeta)=q(\widehat{y})+\left(\xi+\zeta-2 \frac{\xi \zeta}{\xi+\zeta}\right) n^{2} \mathrm{~K}(\widehat{y})+\mathcal{O}\left((\xi+\zeta)^{2}\right) .
$$


From this follows

$$
\left\{\widehat{\Delta}_{\mathrm{b}}\right\}(\widehat{y} ; \xi, \zeta)=1+\left(\xi+\zeta-2 \frac{\xi \zeta}{\xi+\zeta}\right) n^{2} k(\widehat{y})+\mathcal{O}\left((\xi+\zeta)^{2}\right)
$$

Together with

$$
\hat{j}(\widehat{y}, \eta)=1+n^{2} k(\widehat{y}) \eta+\mathcal{O}\left(\eta^{2}\right)
$$

and the definition A.52), it finally gives the expansion for $\tilde{j}_{\mathrm{b}}$,

$$
\tilde{j}_{\mathrm{b}}(\widehat{y} ; \xi, \lambda, \zeta)=1+\left(\frac{\xi+\zeta}{2}-(1-2 p) \lambda\right) n^{2} k(\widehat{y})+\mathcal{O}\left((\xi+\zeta+\lambda)^{2}\right) .
$$




\section{Special functions $\mathbf{R}_{\nu}$}

In appendix A we have used various integrals of exponentials of quadratic exponent and integrals of such integrals. Here we summarize properties of such integrals. We will introduce a special function $\mathrm{R}_{\nu}$ closely related to error functions erfc $(x)$, the definition and properties of which can be found, for example, in [24]. The derivation of the properties below are not all simple, and we do not include them here.

We define the function $\mathrm{R}_{\nu}(x)$ for positive $\nu$ as

$$
\mathrm{R}_{\nu}(z)=\frac{1}{\Gamma(\nu)} \int_{\mathbb{R}^{+}} d x x^{\nu-1} \exp \left(-\frac{1}{2}(x-z)^{2}\right) .
$$

It is a solution of the differential equation

$$
\mathrm{R}_{\nu}^{\prime}(z)=\nu \mathrm{R}_{\nu+1}(z)-z \mathrm{R}_{\nu}(z) \quad, \quad \mathrm{R}_{\nu} \stackrel{z \rightarrow-\infty}{\longrightarrow} 0 .
$$

In the limit $\nu \rightarrow 0$ and for $\nu=1$ we have

$$
\begin{gathered}
\mathrm{R}_{0}(z)=\exp \left(-\frac{1}{2} z^{2}\right) \\
\mathrm{R}_{1}(z)=\sqrt{2 \pi}-\sqrt{\frac{\pi}{2}} \operatorname{erfc}\left(\frac{z}{\sqrt{2}}\right) .
\end{gathered}
$$

We also have the recurrence relation

$$
\mathrm{R}_{\nu+2}(z)=\frac{1}{\nu+1}\left(z \mathrm{R}_{\nu+1}(z)+\mathrm{R}_{\nu}(z)\right) .
$$

For $\nu \in \mathbb{N}$ these functions are combinations of $\mathrm{R}_{0}$ and $\mathrm{R}_{1}$ with polynomial coefficients

$$
\mathrm{R}_{n+1}=\mathrm{p}_{n} \mathrm{R}_{1}+\mathrm{q}_{n-1} \mathrm{R}_{0} \quad \text { for } \quad n \in \mathbb{N} \quad,
$$

where

$$
\begin{gathered}
\mathrm{p}_{n+1}(z)=\frac{1}{n+1}\left(z \mathrm{p}_{n}(z)+\mathrm{p}_{n-1}(z)\right) \quad, \quad \mathrm{p}_{0}=1 \quad, \quad \mathrm{p}_{1}=z \\
\mathrm{q}_{n+1}(z)=\frac{1}{n+2}\left(z \mathrm{q}_{n}(z)+\mathrm{q}_{n-1}(z)\right) \quad, \quad \mathrm{q}_{0}=1 \quad, \quad \mathrm{q}_{1}=\frac{1}{2} z
\end{gathered}
$$

These polynomials satisfy

$$
\mathrm{p}_{n}^{\prime}=\mathrm{p}_{n-1} \quad, \quad \mathrm{q}_{n}^{\prime}=(n+2) \mathrm{q}_{n+1}-\mathrm{p}_{n+1},
$$

and

$$
\sqrt{2 \pi} \mathrm{p}_{n}(z)=\mathrm{R}_{n+1}(z)+(-1)^{n} \mathrm{R}_{n+1}(-z)=\frac{\sqrt{2 \pi}}{i^{n} 2^{\frac{n}{2}} n !} \mathrm{H}_{n}\left(i \frac{z}{\sqrt{2}}\right)
$$


where $\mathrm{H}_{n}$ are the Hermite polynomials (see [24]).

Values at zero are

$$
\begin{aligned}
& \mathrm{R}_{\nu}(0)=2^{\frac{\nu}{2}} \frac{\Gamma\left(\frac{\nu}{2}+1\right)}{\Gamma(\nu+1)}=\frac{1}{2} \frac{\sqrt{2 \pi}}{2^{\frac{\nu-1}{2}} \Gamma\left(\frac{\nu-1}{2}+1\right)}=\left\{\begin{array}{ll}
\frac{1}{(\nu-1) ! !} & \text { for } \nu \text { natural and even } \\
\frac{1}{\nu ! !} \sqrt{\frac{\pi}{2}} & \text { for } \nu \text { natural and odd }
\end{array},\right. \\
& \mathrm{p}_{n}(0)=\left\{\begin{array}{ll}
\frac{1}{n ! !} & \text { for } n \text { even } \\
0 & \text { for } n \text { odd }
\end{array}, \quad \mathrm{p}_{n}^{\prime}=\left\{\begin{array}{ll}
0 & \text { for } n \text { even } \\
\frac{1}{(n-1) ! !} & \text { for } n \text { odd }
\end{array},\right.\right. \\
& \mathrm{q}_{n}(0)=\left\{\begin{array}{ll}
\frac{1}{(n+1) ! !} & \text { for } n \text { even } \\
0 & \text { for } n \text { odd }
\end{array}, \quad \mathrm{q}_{n}^{\prime}=\left\{\begin{array}{ll}
0 & \text { for } n \text { even } \\
\frac{1}{n ! !}-\frac{1}{(n+1) ! !} & \text { for } n \text { odd }
\end{array} .\right.\right.
\end{aligned}
$$

The behavior for small $z$ can be found for natural $\nu$ with help of relations (C.5) and

$$
\begin{aligned}
& \mathrm{R}_{0}(z)=\sum_{k \in \mathbb{N}_{0}} \frac{(-1)^{k}}{(2 k) ! !} z^{2 k} \\
& \mathrm{R}_{1}(z)=\sqrt{\frac{\pi}{2}}+\sum_{k \in \mathbb{N}_{0}} \frac{(-1)^{k}}{(2 k+1)(2 k) ! !} z^{2 k+1} .
\end{aligned}
$$

The behavior for $|z| \gg 1$ and $n \in \mathbb{N}_{0}$ is

$$
\mathrm{R}_{n+1}(z)=\sqrt{2 \pi} \mathrm{p}_{n}(z) \theta(z)+\exp \left(-\frac{1}{2} z^{2}\right) \mathcal{O}\left(\frac{1}{z^{n+1}}\right)
$$

where $\theta(z)$ is the step function.

Now we can write down results of some integrals in terms of these functions. For $n \in \mathbb{N}_{0}$ we have

$$
\frac{1}{n !} \int_{\mathbb{R}} d x x^{n} \exp \left(-\frac{1}{2}(x-z)^{2}\right)=\sqrt{2 \pi} \mathrm{p}_{n}(z)
$$

Further for $k, l \in \mathbb{N}_{0}$ we have

$$
\begin{aligned}
& \frac{1}{l !} \int_{\langle-\infty, x\rangle} d \xi \xi^{l} \mathrm{R}_{k}(\xi)=\sum_{m=0, \ldots, l} \frac{(-1)^{l+m}}{m !} x^{m} \mathrm{R}_{k+l-m+1}(x)= \\
& =\sqrt{2 \pi}(-1)^{l} \mathrm{p}_{k+l}(0)+\frac{\sqrt{2 \pi}}{l !(k-1) !} \sum_{\substack{m \in \mathbb{N} \\
2 m \leqslant k-1}} \frac{(2 m-1) ! !}{k+l-2 m}\left(\begin{array}{c}
k-1 \\
2 m
\end{array}\right) x^{k+l-2 m}- \\
& -\sum_{m=0, \ldots, l} \frac{1}{m !} x^{m} \mathrm{R}_{k+l-m+1}(-x)
\end{aligned},
$$

Finally for $m, k, l \in \mathbb{R}^{+}$and $n=m+k+l$ we have

$$
\frac{1}{n !} \int_{\xi, \zeta \in \mathbb{R}^{+}} d \xi d \zeta \frac{\xi^{m+k} \zeta^{m+l}}{(\xi+\zeta)^{m}} \exp \left(-\frac{1}{2}(\xi+\zeta)^{2}\right)=\sqrt{2 \pi} 2^{-\frac{n+1}{2}} \frac{\Gamma(m+k+1) \Gamma(m+l+1)}{\Gamma(b+m+1) \Gamma\left(\frac{n+1}{2}\right)}
$$




\section{Notes}

1 We use the MTW sign convention ([23]) for spacetime quantities generalized to accomodate both Euclidian and Lorentzian versions of the theory. The version is determined by the constant factor $n$ which is equal to 1 in the Euclidian version or to $i$ in the Lorentzian version. Specifically, geometric quantities defined in spacetime $M$ with an embeded hypersurface $\Sigma$ are related in the following way:

$$
\begin{aligned}
& \text { metric: } \quad g_{\boldsymbol{\alpha} \boldsymbol{\beta}}=n^{2} \mathrm{n}_{\boldsymbol{\alpha}} \mathrm{n}_{\boldsymbol{\beta}}+q_{\boldsymbol{\alpha} \boldsymbol{\beta}} \quad, \quad q \text { positive definite , } \\
& \text { volume element: } \quad \mathfrak{g}^{\frac{1}{2}}=\frac{1}{n}(\operatorname{Det} g)^{\frac{1}{2}} \text {, } \\
& \text { curvature: } \quad \mathrm{R}_{\boldsymbol{\alpha} \boldsymbol{\beta}}{ }_{\boldsymbol{\mu}} a^{\boldsymbol{\nu}}=\nabla_{[\boldsymbol{\alpha}} \nabla_{\boldsymbol{\beta}]} a^{\boldsymbol{\mu}}, \operatorname{Ric}_{\boldsymbol{\alpha} \boldsymbol{\beta}}=\mathrm{R}_{\boldsymbol{\mu} \boldsymbol{\alpha}}{ }^{\boldsymbol{\mu}}{ }_{\boldsymbol{\beta}}, R=\operatorname{Ric}_{\boldsymbol{\alpha} \boldsymbol{\beta}} g^{-1 \boldsymbol{\alpha} \boldsymbol{\beta}} \text {, } \\
& \text { extrinsic curvature: } \mathrm{K}_{\boldsymbol{\alpha} \boldsymbol{\beta}}=\mathfrak{d}_{\boldsymbol{\alpha}}^{\boldsymbol{\mu}} \mathfrak{\jmath}_{\boldsymbol{\beta}}^{\nu} \nabla_{\boldsymbol{\mu}} n_{\boldsymbol{\nu}} \quad, \quad k=\mathrm{K}_{\boldsymbol{\alpha} \boldsymbol{\beta}} g^{-1 \boldsymbol{\alpha} \boldsymbol{\beta}}, \\
& n \text { has the orientation of the hypersurface } \Sigma \text {, } \\
& \text { projector on } \Sigma: \quad \mathfrak{o}_{\boldsymbol{\alpha}}^{\boldsymbol{\beta}}=\delta_{\boldsymbol{\alpha}}^{\boldsymbol{\beta}}-\vec{n}^{\boldsymbol{\beta}} n_{\boldsymbol{\alpha}} \quad, \quad \vec{n}^{\boldsymbol{\alpha}}=n^{2} n_{\boldsymbol{\mu}} g^{-1 \boldsymbol{\mu} \boldsymbol{\alpha}} \text {. }
\end{aligned}
$$

2 Euclidian and physical actions are related by

$$
-I(\mathrm{~h})=\nu S(\mathrm{~h}) \quad .
$$

The version of the theory is determined by actual value of the factor $\nu$ - whether it is real or imaginary.

3 The factor $n$, which governs the signature of the spacetime metric, is chosen here for convenience and reflects that we are using Lorentzian convention for volume element 1 . I.e., the physical amplitude is $\frac{1}{n} K$, but the quantity $K$ will have nicer properties in language of Lorentzian quantities. Similarly, for the Green function the physical amplitude is $\frac{1}{n} G^{F}$, but we will use often the quantity $G^{F}$ to express properties of the amplitude.

4 To abbreviate formulas we often use different dots to indicate contraction in different vector spaces. We use "." for contractions of tangent tensor indices, i.e. $g_{\boldsymbol{\alpha} \boldsymbol{\beta}} a^{\boldsymbol{\beta}}=g \cdot a$. We use "•" for contraction in functional vector spaces of function and densities on spacetime, i.e. the bullet means an integration over spacetime (or an action of a distribution on a test function - a formal integration):

$$
\phi \bullet \alpha=\int_{M} \phi \alpha .
$$

Similarly we use "." for integration over a hypersurface $\Sigma$ or a boundary $\partial \Omega$ of a spacetime domain. 
${ }^{5}$ A volume element $\mathfrak{g}^{\frac{1}{2}}$ on spacetime defines a bi-distribution $\mathcal{G}=\mathfrak{g}^{\frac{1}{2}} \delta$ - a delta function normalized to the volume element, i.e. $\phi \bullet \mathcal{G} \bullet \psi=\int \phi \psi \mathfrak{g}^{\frac{1}{2}}$. Clearly for a smooth function $f$ we can define a distribution $f \mathcal{G}$.

It is convenient to represent differential operators on the manifold $M$ as bi-distributions. We use arrows $m$ and $\rightsquigarrow$ to indicate direction of derivatives. So, for example,

$$
\psi \cdot\left(\tilde{\mathrm{d}}_{\boldsymbol{\alpha}} a^{\boldsymbol{\alpha}}\right) \bullet \omega=\omega \bullet\left(a^{\boldsymbol{\alpha}} \stackrel{\mathrm{d}}{\boldsymbol{\alpha}}\right) \bullet \psi=\int \omega a^{\boldsymbol{\alpha}} \mathrm{d}_{\boldsymbol{\alpha}} \psi
$$

for a test function $\psi$, a test density $\omega$, and a vector field $a$.

${ }^{6}$ A meaning of the tilde in the index of the quadratic form $\mathcal{F}_{\tilde{k}}$ will not be discussed in this paper. It will be explained in the following paper and can be safely ignored here.

7 Again, we factorize out the prefactor $n$ motivated by the fact that $J$ is a density, i.e. proportional to volume element $\mathfrak{g}^{\frac{1}{2}}$. 


\section{References}

[1] R. P. Feynman, R. B. Leighton, and M. L. Sands, The Feynman Lectures on Physics (AddisonWesley, Reading, 1963-65).

[2] R. P. Feynman and A. R. Hibbs, Quantum Mechanics and Path Integrals (McGraw-Hill, New York, 1965).

[3] L. S. Schulman, Techniques and Applications of Path Integration (John Willey \& Sons, Inc., New York, 1981).

[4] B. Simon, Functional Integration and Quantum Physics (Academic Press, New York, 1979).

[5] R. P. Feynman, QED: The Strange Theory of Light and Matter (Princeton University Press, Princeton, N.J., 1985).

[6] J. B. Hartle, Spacetime quantum mechanics and the quantum mechanics of spacetime, UCSBTH92-21, gr-qc/9304006, lectures given at the 1992 Les Houches École d'été, Gravitation et Quantifications.

[7] M. Gell-Mann and J. B. Hartle, Quantum mechanics in the light of quantum cosmology, in Compexity, Entropy and the Physics of Information, SFI Studies in the Sciences of Complexity, Vol. VIII, edited by W. Zurek (Addison-Wesley, Reading, 1990).

[8] J. B. Hartle, The quantum mechanics of closed dystems, in Directions in General Relativity, edited by B. L. Hu, M. P. Ryan, and C. V. Vishveshwara (Cambridge University Press, Cambridge, 1993).

[9] P. Krtouš, Quantized Relativistic Particle and Boundary Conditions, in preparation.

[10] L. Parker, Path integrals for a particle in curved space, Phys. Rev. D 19, 438 (1979).

[11] B. S. DeWitt, Quantum field theory in curved spacetime, Phys. Rep. C19, 295 (1975).

[12] A. M. Polyakov, Gauge Fields and Strings (Harwood Academic, New York, 1987).

[13] P. Krtouš, Relationships between Scalar Field and Relativistic Particle Quantizations, Ph.D. thesis, University of Alberta, Edmonton, Canada, 1997.

[14] M. Reed and B. Simon, Methods of Modern Mathematical Physics (Academic Press, New York, $1972-)$.

[15] B. S. DeWitt and R. W. Brehme, Radiation damping in a gravitational field, Ann. Phys. 9, 220 (1960).

[16] B. S. DeWitt, Dynamical Theory of Groups and Fields (Gordon and Brench, New York, 1965).

[17] S. M. Christensen, Regularization, renormalization, and covariant geodetic point separation, Phys. Rev. D 17, 946 (1978). 
[18] D. M. McAvity and H. Osborn, A DeWitt expansion of the heat kernel for manifolds with a boundary, Class. Quantum Grav. 8, 603 (1990).

[19] D. M. McAvity and H. Osborn, Asymptotic expansion of the heat kernel for generalized boundary conditions, Class. Quantum Grav. 8, 1445 (1991).

[20] D. M. McAvity and H. Osborn, Heat kernel asymptotics for mixed boundary conditions, Class. Quantum Grav. 9, 1983 (1992).

[21] Point splitting calculations Using Mathematica and MathTensor, unpublised, available on WWW: http://otokar.troja.mff.cuni.cz/ krtous/Mathematica/pointsplitting/.

[22] S. M. Christensen, Vacuum expectation value of the stress-energy tensor in an arbitrary curved background: The covariant point-separation method, Phys. Rev. D 14, 2490 (1976).

[23] C. W. Misner, K. S. Thorne, and J. A. Wheeler, Gravitation (Freeman, San Francisco, 1973).

[24] I. S. Gradshtein and I. M. Ryzhik, Table of Integrals, Series, and Products (Academic Press, New York, 1994). 